\title{
An Economical Route to Lamivudine Featuring a Novel Strategy for Stereospecific Assembly
}

David R. Snead,* D. Tyler McQuade, Saeed Ahmad, Rudy Krack, Rodger W. Stringham, Justina M. Burns, Irini Abdiaj, Vijayagopal Gopalsamuthiram, Ryan C. Nelson, B. Frank Gupton

\section{Supporting Information}

\section{Contents}

General:.

Technoeconomic Analysis for Cost of Goods.................................................................................... 3

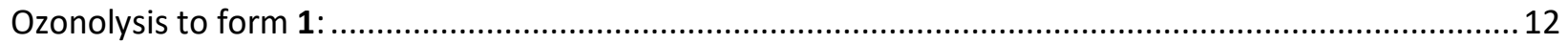

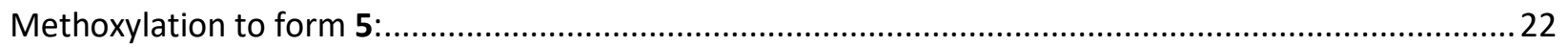

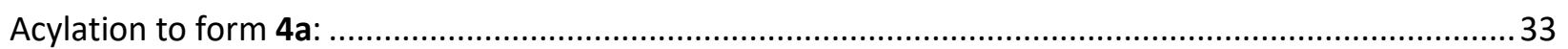

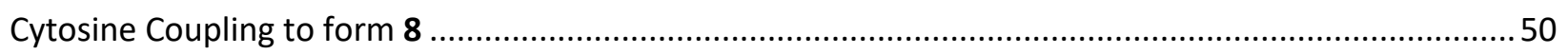

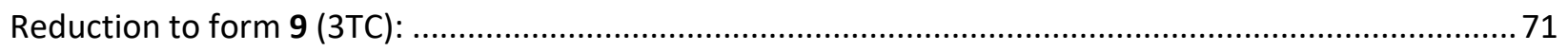




\section{General:}

$1 \mathrm{H}$ NMR spectra were recorded on Bruker $600 \mathrm{MHz}$ spectrometers. Chemical shifts are reported in ppm from tetramethylsilane. Data are reported as follows: chemical shift, integration, multiplicity ( $s=$ singlet, $\mathrm{d}=$ doublet, $\mathrm{t}=$ triplet, $\mathrm{q}=$ quartet, $\mathrm{p}=$ pentet, $\mathrm{h}=$ hextet, hept = heptet, $\mathrm{br}=$ broad, $\mathrm{m}=$ multiplet), and coupling constants $(\mathrm{Hz}) .13 \mathrm{C} \mathrm{NMR}$ was recorded on a Bruker $600 \mathrm{MHz}(151 \mathrm{MHz})$ instrument with complete proton decoupling. Chemical shifts are reported in ppm from tetramethylsilane with the solvent as the internal standard. HRMS was collected using a Jeol AccuTOF-DARTTM mass spectrometer using DART source ionization. Solvents were obtained from VWR or Fisher as HPLC grade unless otherwise specified. Reagents were purchased from Sigma-Aldrich and used as received unless otherwise specified. 


\section{Technoeconomic Analysis for Cost of Goods}

Table S1: Economic analysis of new route as compared to current price of 3TC and CoGs from patent.

\begin{tabular}{|c|c|}
\hline Current 3TC Market Price & $\$ 144 / \mathrm{kg}$ \\
\hline & Cost of Goods \\
\hline Lactic Acid: Current State & $\$ 112 / \mathrm{kg}$ \\
\hline Lactic Acid: Minor Optimization & $\$ 98 / \mathrm{kg}$ \\
\hline Lactic Acid: Aggressive Optimization & $\$ 73 / \mathrm{kg}$ \\
\hline Menthol Route: US8748604 (Patent) & $\$ 170 / \mathrm{kg}$ \\
\hline
\end{tabular}

\section{Assumptions:}

\section{Lactic Acid: Current State}

o $75 \%$ Solvent recycle

o Make (S)-2-chloropropionic acid from alanine

- $\quad$ For reference see: a) Raza, A.F.; Saddiza, A.; Cakmak, O. Chirality, 2015, 27, 951957. b) Ding, Y.; Yuan, Y.; Wang, F.; Jiang, J.; Chen, X. CN107879925, 2017.

- Lab results adapted from Raza et al. (Alternatively can be purchased for $\$ 10 / \mathrm{kg}$ )

\section{Lactic Acid: Minor Optimization}

o Change $4 \mathrm{M} \mathrm{HCl}$ in dioxane to sulfuric acid in Step 3 (methoxylation)

o Improve costing related to sodium methoxide reagent package and solvent recycle Step 3 (methoxylation)

o Change potassium carbonate to sodium carbonate in Step 4 (acylation)

\section{Lactic Acid: Aggressive Optimization}

o Increase solvent recycle to $90 \%$

- Decrease reagent consumption in Step 4 (acylation)

o Increase yield from $54 \%$ to $65 \%$ in Step 4 (acylation)

o Reduce consumption of sodium borohydride to 1.3 equivalents in Step 6 (reduction) 


\section{Lactic Acid: Current State}

Table S2: Cost of Goods (CoGs) for Lactic acid route exactly as described in manuscript.

\begin{tabular}{|c|c|c|c|c|c|c|c|c|c|c|c|c|c|}
\hline Raw materials & Mol. Wt. & Amount & Amount & Mole & equivalent & density & CAS\# & RM Cost & RM Cost & RM Cost & & kg RM / & \% RM Cost/ \\
\hline & & & $/ \mathrm{kg}$ product & & & & & $\$ / \mathrm{kg}$ & $\$ /$ batch & $\$ / \mathrm{kg}$ product & kg RM & kg prod & Total RM cost \\
\hline \multicolumn{14}{|l|}{ Step 1} \\
\hline Dimethyl Maleate & 144 & 144.00 & 0.82 & 1 & 1 & & $922-68-9$ & 2.70 & 388.80 & 2.22 & 144.00 & & $1.97 \%$ \\
\hline 1,4-Dithianediol & 152.2 & 152.20 & 0.87 & 1 & 1 & & $40018-26-6$ & 9.90 & $1,506.78$ & 8.60 & 152.20 & & $7.65 \%$ \\
\hline Dimethyl Sulfide & 62.13 & 80.77 & 0.46 & & 1.3 & & \begin{tabular}{|l|}
$75-18-3$ \\
\end{tabular} & 1.53 & 123.25 & 0.70 & 80.77 & & $0.63 \%$ \\
\hline Dichloromethane & 84.93 & 477.00 & 2.72 & & $10 \mathrm{~V}$ & 1.325 & $75-09-2$ & 0.68 & 324.36 & 1.85 & 477.00 & & $1.65 \%$ \\
\hline Acetonitrile & 41.05 & 141.48 & 0.81 & & $5 \mathrm{~V}$ & 0.786 & $75-05-8$ & 0.97 & 137.24 & 0.78 & 141.48 & & $0.70 \%$ \\
\hline \multicolumn{14}{|l|}{ Product } \\
\hline $84 \%$ & 164 & 275.52 & 1.57 & & & & & & $2,480.43$ & 9.00 & 995.45 & 3.61 & $12.59 \%$ \\
\hline \multicolumn{14}{|l|}{ Step 2} \\
\hline Alanine & 89.09 & 89.09 & 2.02 & 1.00 & 1.00 & & $56-41-7$ & 2.07 & 184.42 & 4.19 & 89.09 & & $3.72 \%$ \\
\hline Sodium Nitrite & 69 & 231.84 & 5.26 & 3.36 & 3.36 & & $7632-00-0$ & 0.53 & 122.88 & 2.79 & 231.84 & & $2.48 \%$ \\
\hline Concentrated $\mathrm{HCl}$ (aq.) & 36.46 & $1,260.45$ & 28.61 & & $12 \mathrm{~V}$ & 1.179 & $7647-01-0$ & 0.10 & 126.04 & 2.86 & $1,260.45$ & & $2.55 \%$ \\
\hline \multicolumn{14}{|l|}{ Product } \\
\hline $80 \%$ & 108.5 & 86.80 & 1.97 & & & & & & 433.34 & 4.99 & $1,581.38$ & 18.22 & $8.75 \%$ \\
\hline \multicolumn{14}{|l|}{ Step 3} \\
\hline Step 2 Product & 108.5 & 108.50 & 1.97 & 1 & 1 & & $29617-66-1$ & 4.99 & 541.67 & 9.84 & $1,976.72$ & & \\
\hline $25 \mathrm{wt} \% \mathrm{NaOMe}$ in $\mathrm{MeOH}$ (3 equiv.) & 104.1 & 312.30 & 5.67 & 3 & 3 & 0.945 & 124-41-4 & 0.79 & 246.72 & 4.48 & 312.30 & & $3.99 \%$ \\
\hline Methanol for NaOMe solution & 32.04 & 312.30 & 5.67 & & & 0.791 & $67-56-1$ & 0.42 & 131.17 & 2.38 & 312.30 & & $2.12 \%$ \\
\hline $4 \mathrm{M} \mathrm{HCl}$ in Dioxane & 36.46 & 36.46 & 0.66 & & 1 & & 7647-01-0 & 3.74 & 136.36 & 2.48 & 36.46 & & $2.20 \%$ \\
\hline 1,4-Dioxane for $4 \mathrm{M} \mathrm{HCl}$ solution & 88.106 & 65.11 & 1.18 & & & 1.036 & \begin{tabular}{|l|}
$123-91-1$ \\
\end{tabular} & 2.50 & 162.77 & 2.96 & 65.11 & & $2.63 \%$ \\
\hline Toluene & 92.134 & 70.55 & 1.28 & & $3 \mathrm{~V}$ & 0.867 & $108-88-3$ & 0.60 & 42.33 & 0.77 & 70.55 & & $0.68 \%$ \\
\hline \multicolumn{14}{|l|}{ Product } \\
\hline $95 \%$ & 126 & 119.70 & 2.17 & & & & & & $1,261.01$ & 10.53 & $2,773.44$ & 23.17 & $11.62 \%$ \\
\hline \multicolumn{14}{|l|}{ Step 4} \\
\hline Step 3 Product & 126 & 226.80 & 2.17 & 1.8 & 1.8 & & & 10.53 & $2,389.29$ & 22.90 & $5,254.94$ & & \\
\hline Step 1 Product & 164 & 164.00 & 1.57 & 1 & 1 & & & 9.00 & $1,476.45$ & 14.15 & 592.53 & & \\
\hline Pivaloyl Chloride & 120.6 & 217.08 & 2.08 & 1.8 & 1.8 & 0.979 & $3282-30-2$ & 2.75 & 596.97 & 5.72 & 217.08 & & $5.09 \%$ \\
\hline Levamisole & 122.2 & 1.83 & 0.02 & 0.015 & 0.015 & & $1122-58-3$ & 24.50 & 44.91 & 0.43 & 1.83 & & $0.38 \%$ \\
\hline 2-methylpyridine & 93 & 18.60 & 0.18 & 0.2 & 0.2 & & $109-06-8$ & 2.50 & 46.50 & 0.45 & 18.60 & & $0.40 \%$ \\
\hline $\mathrm{DCM}$ & 84.93 & 375.64 & 3.60 & & $10 \mathrm{~V}$ & 1.325 & $75-09-2$ & 0.68 & 255.43 & 2.45 & 375.64 & & $2.18 \%$ \\
\hline $1 \mathrm{M} \mathrm{HCl}$ (Aqueous) & 36.46 & 29.52 & 0.28 & & $5 \mathrm{~V}$ & & 7647-01-0 & 0.75 & 22.14 & 0.21 & 29.52 & & $0.19 \%$ \\
\hline Water for $1 \mathrm{M} \mathrm{HCl}$ solution & 18.015 & 820.00 & 7.86 & & & 1 & & 0.06 & 49.20 & 0.47 & 820.00 & & $0.42 \%$ \\
\hline Water & 18.015 & 820.00 & 7.86 & & $5 \mathrm{~V}$ & 1 & & 0.06 & 49.20 & 0.47 & 820.00 & & $0.42 \%$ \\
\hline Saturated $\mathrm{K} 2 \mathrm{CO} 3$ & 105.988 & 192.70 & 1.85 & & $5 \mathrm{~V}$ & & $497-19-8$ & 1.45 & 279.42 & 2.68 & 192.70 & & $2.38 \%$ \\
\hline Water for $\mathrm{K} 2 \mathrm{CO} 3$ solution & 18.015 & 820.00 & 7.86 & & & 1 & & 0.06 & 49.20 & 0.47 & 820.00 & & $0.42 \%$ \\
\hline Toluene & 92.134 & 177.74 & 1.70 & & $1.5 \mathrm{~V} ?$ & 0.867 & $108-88-3$ & 0.60 & 106.64 & 1.02 & 177.74 & & $0.91 \%$ \\
\hline Hexanes & 86.178 & 107.42 & 1.03 & & $4 \mathrm{~V}$ & 0.655 & $110-54-3$ & 1.00 & 107.42 & 1.03 & 107.42 & & $0.92 \%$ \\
\hline \multicolumn{14}{|l|}{ Product } \\
\hline $54.0 \%$ & 250 & 135.00 & 1.29 & & & & & & $5,472.76$ & 40.54 & $9,427.99$ & 69.84 & $13.70 \%$ \\
\hline
\end{tabular}




\begin{tabular}{|c|c|c|c|c|c|c|c|c|c|c|c|c|c|}
\hline Step 5 & & & & & & & & & & & & & \\
\hline Step 4 Product & 250 & 250.00 & 1.29 & 1 & 1 & & & 40.54 & $10,134.74$ & 52.45 & $17,459.24$ & & \\
\hline Cytosine & 111 & 166.50 & 0.86 & 1.5 & 1.5 & & 14631-20-0 & 27.00 & $4,495.50$ & 23.27 & \begin{tabular}{|l|}
166.50 \\
\end{tabular} & & $20.70 \%$ \\
\hline Bromine & 159 & 238.50 & 1.23 & 1.5 & 1.5 & & \begin{tabular}{|l|}
$7726-95-6$ \\
\end{tabular} & 2.90 & 691.65 & 3.58 & 238.50 & & $3.18 \%$ \\
\hline Mesitylene & 120.2 & 240.40 & 1.24 & 2 & 2 & 0.864 & 63148-57-2 & 3.00 & 721.20 & 3.73 & 240.40 & & $3.32 \%$ \\
\hline HMDS & 161.4 & 274.38 & 1.42 & 1.7 & 1.7 & & 10416-59-8 & 3.00 & 823.14 & 4.26 & 274.38 & & $3.79 \%$ \\
\hline Tributylamine & 185 & 277.50 & 1.44 & 1.5 & 1.5 & & \begin{tabular}{|l|}
$102-82-9$ \\
\end{tabular} & 1.40 & 388.50 & 2.01 & 277.50 & & $1.79 \%$ \\
\hline Dichloromethane & 84.93 & $1,656.25$ & 8.57 & & $10 \mathrm{~V}$ & 1.325 & $75-09-2$ & 0.68 & $1,126.25$ & 5.83 & $1,656.25$ & & $5.19 \%$ \\
\hline Methanol & 32.04 & 197.75 & 1.02 & & $4 \mathrm{~V}$ & 0.791 & $67-56-1$ & 0.42 & 83.06 & 0.43 & 197.75 & & $0.38 \%$ \\
\hline Hexanes & 86.178 & 163.75 & 0.85 & & $4 \mathrm{~V}$ & 0.655 & $110-54-3$ & 1.00 & 163.75 & 0.85 & 163.75 & & $0.75 \%$ \\
\hline \multicolumn{14}{|l|}{ Product } \\
\hline $86.0 \%$ & 257 & 221.02 & 1.14 & & & & & & $18,627.79$ & 84.28 & $20,674.27$ & 93.54 & $39.10 \%$ \\
\hline \multicolumn{14}{|l|}{ Step 6} \\
\hline Step 5 Product & 257 & 257.00 & 1.14 & 1 & 1 & & & 84.28 & $21,660.22$ & 96.41 & $24,039.85$ & & \\
\hline Sodium borohydride & 37.83 & 68.09 & 0.30 & 1.8 & 1.8 & & $16940-66-2$ & 26.60 & $1,811.30$ & 8.06 & \begin{tabular}{|l|}
68.09 \\
\end{tabular} & & $7.17 \%$ \\
\hline EtOH & 32.04 & 508.86 & 2.26 & & $10 \mathrm{~V}$ & 0.792 & $64-17-5$ & 0.91 & 463.06 & 2.06 & 508.86 & & $1.83 \%$ \\
\hline EtOAc & 88.106 & $1,159.07$ & 5.16 & & $20 \mathrm{~V}$ & 0.902 & $141-78-6$ & 0.84 & 973.62 & 4.33 & $1,159.07$ & & $3.86 \%$ \\
\hline Oxalic Acid & 90.03 & 360.12 & 1.60 & & 4 & & $144-62-77$ & 0.50 & 180.06 & 0.80 & 360.12 & & $0.71 \%$ \\
\hline $3 \mathrm{M} \mathrm{HCl}$ (aq.) & 36.46 & 56.54 & 0.25 & & $2 \mathrm{~V}$ & & $7647-01-0$ & 0.75 & 42.41 & 0.19 & 56.54 & & $0.17 \%$ \\
\hline Water for $3 \mathrm{M} \mathrm{HCl}$ solution & 18.015 & 514.00 & 2.29 & & & 1 & & 0.06 & 30.84 & 0.14 & 514.00 & & $0.12 \%$ \\
\hline $45 \% \mathrm{NaOH}$ (aq.) & 40 & 194.68 & 0.87 & & $0.5 \mathrm{~V}$ & 1.515 & $1310-73-2$ & 0.48 & 93.45 & 0.42 & 194.68 & & $0.37 \%$ \\
\hline \multicolumn{14}{|l|}{ Product } \\
\hline \multirow[t]{2}{*}{$98.0 \%$} & 229.26 & 224.67 & 1.00 & & & & & & $25,254.95$ & 112.41 & $26,901.21$ & 119.73 & $14.23 \%$ \\
\hline & & & & & & & & & & $\$ / \mathbf{k g}$ & & PMI & $100.00 \%$ \\
\hline \multirow[t]{2}{*}{ Overall yield } & $29.05 \%$ & & & & & & & & & & & & \\
\hline & & & & & & & & & Total RM Cost $\$$ & 112.41 & & PMI & Sum of steps \\
\hline \multirow[t]{2}{*}{ Solvent recycle } & $75 \%$ & & & & & & & & & & & 119.73 & $\%$ of total \\
\hline & & & & & & & & & & & & & $100.00 \%$ \\
\hline
\end{tabular}

\section{Assumptions:}

\section{Lactic Acid: Current State}

$0 \quad 75 \%$ Solvent recycle

o Make (S)-2-chloropropionic acid from alanine

- For reference see: a) Raza, A.F.; Saddiza, A.; Cakmak, O. Chirality, 2015, 27, 951-957. b) Ding, Y.; Yuan, Y.; Wang, F.; Jiang, J.; Chen, X. CN107879925, 2017.

- Lab results adapted from Raza et al. (Alternatively can be purchased for $\$ 10 / \mathrm{kg}$ ) 


\section{Lactic Acid: Minor Optimization}

Table S3: Cost of Goods (CoGs) for Lactic acid route as described in manuscript with minor optimization.

\begin{tabular}{|c|c|c|c|c|c|c|c|c|c|c|c|c|c|}
\hline Raw materials & Mol. Wt. & Amount & Amount & Mole & \begin{tabular}{|l|} 
equivalent \\
\end{tabular} & density & CAS\# & RM Cost & RM Cost & RM Cost & & kg RM / & \% RM Cost/ \\
\hline & & & / kg product & & & & & $\$ / \mathbf{k g}$ & \$ / batch & $\$ / \mathrm{kg}$ product & kg RM & kg prod & Total RM cost \\
\hline \multicolumn{14}{|l|}{ Step 1} \\
\hline Dimethyl Maleate & 144 & 144.00 & 0.82 & 1 & 1 & & $922-68-9$ & 2.70 & 388.80 & 2.22 & 144.00 & & $2.26 \%$ \\
\hline 1,4-Dithianediol & 152.2 & 152.20 & 0.87 & 1 & 1 & & 40018-26-6 & 9.90 & $1,506.78$ & 8.60 & 152.20 & & $8.74 \%$ \\
\hline Dimethyl Sulfide & 62.13 & 80.77 & 0.46 & & 1.3 & & $75-18-3$ & 1.53 & 123.25 & 0.70 & 80.77 & & $0.71 \%$ \\
\hline Dichloromethane & 84.93 & 477.00 & 2.72 & & $10 \mathrm{~V}$ & 1.325 & $75-09-2$ & 0.68 & 324.36 & 1.85 & 477.00 & & $1.88 \%$ \\
\hline Acetonitrile & 41.05 & 141.48 & 0.81 & & $5 \mathrm{~V}$ & 0.786 & $75-05-8$ & 0.97 & 137.24 & 0.78 & 141.48 & & $0.80 \%$ \\
\hline \multicolumn{14}{|l|}{ Product } \\
\hline $84 \%$ & 164 & 275.52 & 1.57 & & & & & & $2,480.43$ & 9.00 & 995.45 & 3.61 & $14.39 \%$ \\
\hline \multicolumn{14}{|l|}{ Step 2} \\
\hline Alanine & 89.09 & 89.09 & 2.02 & 1.00 & 1.00 & & $56-41-7$ & 2.07 & 184.42 & 4.19 & 89.09 & & $4.26 \%$ \\
\hline Sodium Nitrite & 69 & 231.84 & 5.26 & 3.36 & 3.36 & & $7632-00-0$ & 0.53 & 122.88 & 2.79 & 231.84 & & $2.84 \%$ \\
\hline Concentrated $\mathrm{HCl}$ (aq.) & 36.46 & $1,260.45$ & 28.61 & & $12 \mathrm{~V}$ & 1.179 & 7647-01-0 & 0.10 & 126.04 & 2.86 & $1,260.45$ & & $2.91 \%$ \\
\hline \multicolumn{14}{|l|}{ Product } \\
\hline $80 \%$ & 108.5 & 86.80 & 1.97 & & & & & & 433.34 & 4.99 & $1,581.38$ & 18.22 & $10.00 \%$ \\
\hline \multicolumn{14}{|l|}{ Step 3} \\
\hline Step 2 Product & 108.5 & 108.50 & 1.97 & 1 & 1 & & $29617-66-1$ & 4.99 & 541.67 & 9.84 & $1,976.72$ & & \\
\hline Sodium Metal & 23 & 69.00 & 1.25 & 3 & 3 & & & 2.80 & 193.20 & 3.51 & 69.00 & & $3.57 \%$ \\
\hline Methanol for $\mathrm{NaOMe}$ solution & 32.04 & 153.80 & 2.79 & & & 0.791 & $67-56-1$ & 0.42 & 64.60 & 1.17 & 153.80 & & $1.19 \%$ \\
\hline Sulfuric Acid & 98 & 98.00 & 1.78 & & 1 & & 7647-01-0 & 0.05 & 4.90 & 0.09 & 98.00 & & $0.09 \%$ \\
\hline Toluene & 92.134 & 70.55 & 1.28 & & $3 \mathrm{~V}$ & 0.867 & $108-88-3$ & 0.60 & 42.33 & 0.77 & 70.55 & & $0.78 \%$ \\
\hline \multicolumn{14}{|l|}{ Product } \\
\hline $95 \%$ & 126 & 119.70 & 2.17 & & & & & & 846.70 & 7.07 & $2,368.07$ & 19.78 & $5.63 \%$ \\
\hline \multicolumn{14}{|l|}{ Step 4} \\
\hline Step 3 Product & 126 & 226.80 & 2.17 & 1.8 & 1.8 & & & 7.07 & $1,604.27$ & 15.38 & $4,486.87$ & & \\
\hline Step 1 Product & 164 & 164.00 & 1.57 & 1 & 1 & & & 9.00 & $1,476.45$ & 14.15 & 592.53 & & \\
\hline Pivaloyl Chloride & 120.6 & 217.08 & 2.08 & 1.8 & 1.8 & 0.979 & $3282-30-2$ & 2.75 & 596.97 & 5.72 & 217.08 & & $5.82 \%$ \\
\hline Levamisole & 122.2 & 1.83 & 0.02 & 0.015 & 0.015 & & $1122-58-3$ & 24.50 & 44.91 & 0.43 & 1.83 & & $0.44 \%$ \\
\hline 2-methylpyridine & 93 & 18.60 & 0.18 & 0.2 & 0.2 & & $109-06-8$ & 2.50 & 46.50 & 0.45 & 18.60 & & $0.45 \%$ \\
\hline DCM & 84.93 & 375.64 & 3.60 & & $10 \mathrm{~V}$ & 1.325 & $75-09-2$ & 0.68 & 255.43 & 2.45 & 375.64 & & $2.49 \%$ \\
\hline $1 \mathrm{M} \mathrm{HCl}$ (Aqueous) & 36.46 & 29.52 & 0.28 & & $5 \mathrm{~V}$ & & $7647-01-0$ & 0.10 & 2.95 & 0.03 & 29.52 & & $0.03 \%$ \\
\hline Water for $1 \mathrm{M} \mathrm{HCl}$ solution & 18.015 & 820.00 & 7.86 & & & 1 & & 0.06 & 49.20 & 0.47 & 820.00 & & $0.48 \%$ \\
\hline Water & 18.015 & 820.00 & 7.86 & & $5 \mathrm{~V}$ & 1 & & 0.06 & 49.20 & 0.47 & 820.00 & & $0.48 \%$ \\
\hline Sodium Carbonate & 105.988 & 317.96 & 3.05 & 3 & 3 & & & 0.22 & 69.95 & 0.67 & 317.96 & & $0.68 \%$ \\
\hline Water for $\mathrm{Na} 2 \mathrm{CO} 3$ solution & 18.015 & 820.00 & 7.86 & & & 1 & & 0.06 & 49.20 & 0.47 & 820.00 & & $0.48 \%$ \\
\hline Toluene & 92.134 & 177.74 & 1.70 & & $1.5 \mathrm{~V} ?$ & 0.867 & $108-88-3$ & 0.60 & 106.64 & 1.02 & 177.74 & & $1.04 \%$ \\
\hline Hexanes & 86.178 & 107.42 & 1.03 & & $4 \mathrm{~V}$ & 0.655 & $110-54-3$ & 1.00 & 107.42 & 1.03 & 107.42 & & $1.05 \%$ \\
\hline \multicolumn{14}{|l|}{ Product } \\
\hline $54.0 \%$ & 250 & 135.00 & 1.29 & & & & & & $4,459.09$ & 33.03 & $8,785.19$ & 65.08 & $13.43 \%$ \\
\hline
\end{tabular}




\begin{tabular}{|c|c|c|c|c|c|c|c|c|c|c|c|c|c|}
\hline Step 5 & & & & & & & & & & & & & \\
\hline Step 4 Product & 250 & 250.00 & 1.29 & 1 & 1 & & & 33.03 & $8,257.58$ & 42.74 & $16,268.87$ & & \\
\hline Cytosine & 111 & 166.50 & 0.86 & 1.5 & 1.5 & & 14631-20-0 & 27.00 & $4,495.50$ & 23.27 & 166.50 & & $23.65 \%$ \\
\hline Bromine & 159 & 238.50 & 1.23 & 1.5 & 1.5 & & \begin{tabular}{|l}
$7726-95-6$ \\
\end{tabular} & 2.90 & 691.65 & 3.58 & 238.50 & & $3.64 \%$ \\
\hline Mesitylene & 120.2 & 240.40 & 1.24 & 2 & 2 & 0.864 & 63148-57-2 & 3.00 & 721.20 & 3.73 & 240.40 & & $3.79 \%$ \\
\hline HMDS & 161.4 & 274.38 & 1.42 & 1.7 & 1.7 & & 10416-59-8 & 3.00 & 823.14 & 4.26 & 274.38 & & $4.33 \%$ \\
\hline Tributylamine & 185 & 277.50 & 1.44 & 1.5 & 1.5 & & \begin{tabular}{|l|}
$102-82-9$ \\
\end{tabular} & 1.40 & 388.50 & 2.01 & 277.50 & & $2.04 \%$ \\
\hline Dichloromethane & 84.93 & $1,656.25$ & 8.57 & & $10 \mathrm{~V}$ & 1.325 & $75-09-2$ & 0.68 & $1,126.25$ & 5.83 & $1,656.25$ & & $5.93 \%$ \\
\hline Methanol & 32.04 & 197.75 & 1.02 & & $4 \mathrm{~V}$ & 0.791 & $67-56-1$ & 0.42 & 83.06 & 0.43 & 197.75 & & $0.44 \%$ \\
\hline Hexanes & 86.178 & 163.75 & 0.85 & & $4 \mathrm{~V}$ & 0.655 & $110-54-3$ & 1.00 & 163.75 & 0.85 & 163.75 & & $0.86 \%$ \\
\hline \multicolumn{14}{|l|}{ Product } \\
\hline $86.0 \%$ & 257 & 221.02 & 1.14 & & & & & & $16,750.62$ & 75.79 & $19,483.90$ & 88.15 & $44.69 \%$ \\
\hline \multicolumn{14}{|l|}{ Step 6} \\
\hline Step 5 Product & 257 & 257.00 & 1.14 & 1 & 1 & & & 75.79 & $19,477.47$ & 86.69 & $22,655.69$ & & \\
\hline Sodium borohydride & 37.83 & 68.09 & 0.30 & 1.8 & 1.8 & & $16940-66-2$ & 26.60 & $1,811.30$ & 8.06 & 68.09 & & $8.20 \%$ \\
\hline $\mathrm{EtOH}$ & 32.04 & 508.86 & 2.26 & & $10 \mathrm{~V}$ & 0.792 & $64-17-5$ & 0.91 & 463.06 & 2.06 & 508.86 & & $2.10 \%$ \\
\hline Oxalic Acid & 90.03 & 360.12 & 1.60 & & 4 & & \begin{tabular}{|l|}
$144-62-77$ \\
\end{tabular} & 0.50 & 180.06 & 0.80 & 360.12 & & $0.81 \%$ \\
\hline $3 \mathrm{M} \mathrm{HCl}$ (aq.) & 36.46 & 56.54 & 0.25 & & $2 \mathrm{~V}$ & & $7647-01-0$ & 0.75 & 42.41 & 0.19 & 56.54 & & $0.19 \%$ \\
\hline Water for $3 \mathrm{M} \mathrm{HCl}$ solution & 18.015 & 514.00 & 2.29 & & & 1 & & 0.06 & 30.84 & 0.14 & 514.00 & & $0.14 \%$ \\
\hline $45 \% \mathrm{NaOH}$ (aq.) & 40 & 194.68 & 0.87 & & $0.5 \mathrm{~V}$ & 1.515 & $1310-73-2$ & 0.48 & 93.45 & 0.42 & 194.68 & & $0.42 \%$ \\
\hline \multicolumn{14}{|l|}{ Product } \\
\hline \multirow[t]{2}{*}{$98.0 \%$} & 229.26 & 224.67 & 1.00 & & & & & & $22,098.58$ & 98.36 & $24,357.99$ & 108.41 & $11.86 \%$ \\
\hline & & & & & & & & & & $\$ / \mathbf{k g}$ & & PMI & $100.00 \%$ \\
\hline \multirow[t]{2}{*}{ Overall yield } & $29.05 \%$ & & & & & & & & & & & & \\
\hline & & & & & & & & & Total RM Cost \$ & 98.36 & & PMI & Sum of steps \\
\hline Solvent recycle & $75 \%$ & & & & & & & & & & & 108.41 & $\%$ of total \\
\hline
\end{tabular}

\section{Assumptions:}

\section{Lactic Acid: Minor Optimization}

o Change $4 \mathrm{M} \mathrm{HCl}$ in dioxane to sulfuric acid in Step 3 (methoxylation)

o Improve costing related to sodium methoxide reagent package and solvent recycle Step 3 (methoxylation)

o Change potassium carbonate to sodium carbonate in Step 4 (acylation) 


\section{Lactic Acid: Aggressive Optimization}

Table S4: Cost of Goods (CoGs) for Lactic acid route as described in manuscript with aggressive optimization.

\begin{tabular}{|c|c|c|c|c|c|c|c|c|c|c|c|c|c|}
\hline Raw materials & Mol. Wt. & Amount & Amount & Mole & quivaler & density & CAS\# & RM Cost & RM Cost & RM Cost & & $\mathrm{kg} \mathrm{RM} /$ / & \% RM Cost/ \\
\hline & & \multicolumn{3}{|c|}{ / kg product } & & & & $\$ / \mathbf{k g}$ & $\$ /$ batch & $\$$ / kg product & kg RM & kg prod & Total RM cost \\
\hline \multicolumn{14}{|l|}{ Step 1} \\
\hline Dimethyl Maleate & 144 & 144.00 & 0.68 & 1 & 1 & & $922-68-9$ & 2.70 & 388.80 & 1.84 & 144.00 & & $2.53 \%$ \\
\hline 1,4-Dithianediol & 152.2 & 152.20 & 0.72 & 1 & 1 & & |40018-26-6 & 9.90 & $1,506.78$ & 7.14 & 152.20 & & $9.80 \%$ \\
\hline Dimethyl Sulfide & 62.13 & 80.77 & 0.38 & & 1.3 & & $75-18-3$ & 1.53 & 123.25 & 0.58 & 80.77 & & $0.80 \%$ \\
\hline Dichloromethane & 84.93 & 190.80 & 0.90 & & $10 \mathrm{~V}$ & 1.325 & $75-09-2$ & 0.68 & 129.74 & 0.61 & 190.80 & & $0.84 \%$ \\
\hline Acetonitrile & 41.05 & 56.59 & 0.27 & & $5 \mathrm{~V}$ & 0.786 & $75-05-8$ & 0.97 & 54.89 & 0.26 & 56.59 & & $0.36 \%$ \\
\hline \multicolumn{14}{|l|}{ Product } \\
\hline $84 \%$ & 164 & 275.52 & 1.31 & & & & & & $2,203.47$ & 8.00 & 624.36 & 2.27 & $14.33 \%$ \\
\hline \multicolumn{14}{|l|}{ Step 2} \\
\hline Alanine & 89.09 & 89.09 & 1.31 & 1.00 & 1.00 & & $56-41-7$ & 2.07 & 184.42 & 2.70 & 89.09 & & $3.71 \%$ \\
\hline Sodium Nitrite & 69 & 231.84 & 3.40 & 3.36 & 3.36 & & $7632-00-0$ & 0.53 & 122.88 & 1.80 & 231.84 & & $2.47 \%$ \\
\hline Concentrated $\mathrm{HCl}$ (aq.) & 36.46 & $1,260.45$ & 18.49 & & $12 \mathrm{~V}$ & 1.179 & 7647-01-0 & 0.10 & 126.04 & 1.85 & $1,260.45$ & & $2.54 \%$ \\
\hline \multicolumn{14}{|l|}{ Product } \\
\hline $80 \%$ & 108.5 & 86.80 & 1.27 & & & & & & 433.34 & 4.99 & $1,581.38$ & 18.22 & $8.72 \%$ \\
\hline \multicolumn{14}{|l|}{ Step 3} \\
\hline Step 2 Product & 108.5 & 108.50 & 1.27 & 1 & 1 & & 29617-66-1 & 4.99 & 541.67 & 6.36 & $1,976.72$ & & \\
\hline Sodium Metal & 23 & 69.00 & 0.81 & 3 & 3 & & & 2.80 & 193.20 & 2.27 & 69.00 & & $3.11 \%$ \\
\hline Methanol for $\mathrm{NaOMe}$ solution & 32.04 & 61.52 & 0.72 & & & 0.791 & $67-56-1$ & 0.42 & 25.84 & 0.30 & 61.52 & & $0.42 \%$ \\
\hline Sulfuric Acid & 98 & 98.00 & 1.15 & & 1 & & 7647-01-0 & 0.05 & 4.90 & 0.06 & 98.00 & & $0.08 \%$ \\
\hline Toluene & 92.134 & 28.22 & 0.33 & & $3 \mathrm{~V}$ & 0.867 & $108-88-3$ & 0.60 & 16.93 & 0.20 & 28.22 & & $0.27 \%$ \\
\hline \multicolumn{14}{|l|}{ Product } \\
\hline $95 \%$ & 126 & 119.70 & 1.40 & & & & & & 782.54 & 6.54 & $2,233.46$ & 18.66 & $3.88 \%$ \\
\hline \multicolumn{14}{|l|}{ Step 4} \\
\hline Step 3 Product & 126 & 176.40 & 1.40 & 1.4 & 1.4 & & & 6.54 & $1,153.22$ & 9.18 & $3,291.41$ & & \\
\hline Step 1 Product & 164 & 164.00 & 1.31 & 1 & 1 & & & 8.00 & $1,311.59$ & 10.44 & 371.64 & & \\
\hline Pivaloyl Chloride & 120.6 & 168.84 & 1.34 & 1.4 & 1.4 & 0.979 & $3282-30-2$ & 2.75 & 464.31 & 3.70 & 168.84 & & $5.07 \%$ \\
\hline Levamisole & 122.2 & 1.83 & 0.01 & 0.015 & 0.015 & & $1122-58-3$ & 24.50 & 44.91 & 0.36 & 1.83 & & $0.49 \%$ \\
\hline 2-methylpyridine & 93 & 18.60 & 0.15 & 0.2 & 0.2 & & $109-06-8$ & 2.50 & 46.50 & 0.37 & 18.60 & & $0.51 \%$ \\
\hline DCM & 84.93 & 116.87 & 0.93 & & $10 \mathrm{~V}$ & 1.325 & $75-09-2$ & 0.68 & 79.47 & 0.63 & 116.87 & & $0.87 \%$ \\
\hline $1 \mathrm{M} \mathrm{HCl}$ (Aqueous) & 36.46 & 29.52 & 0.24 & & $5 \mathrm{~V}$ & & 7647-01-0 & 0.10 & 2.95 & 0.02 & 29.52 & & $0.03 \%$ \\
\hline Water for $1 \mathrm{M} \mathrm{HCl}$ solution & 18.015 & 820.00 & 6.53 & & & 1 & & 0.06 & 49.20 & 0.39 & 820.00 & & $0.54 \%$ \\
\hline Water & 18.015 & 820.00 & 6.53 & & $5 \mathrm{~V}$ & 1 & & 0.06 & 49.20 & 0.39 & 820.00 & & $0.54 \%$ \\
\hline Sodium Carbonate & 105.988 & 317.96 & 2.53 & 3 & 3 & & & 0.22 & 69.95 & 0.56 & 317.96 & & $0.76 \%$ \\
\hline Water for $\mathrm{Na} 2 \mathrm{CO} 3$ solution & 18.015 & 820.00 & 6.53 & & & 1 & & 0.06 & 49.20 & 0.39 & 820.00 & & $0.54 \%$ \\
\hline Toluene & 92.134 & 71.09 & 0.57 & & $1.5 \mathrm{~V} ?$ & 0.867 & $108-88-3$ & 0.60 & 42.66 & 0.34 & 71.09 & & $0.47 \%$ \\
\hline Hexanes & 86.178 & 42.97 & 0.34 & & $4 \mathrm{~V}$ & 0.655 & $110-54-3$ & 1.00 & 42.97 & 0.34 & 42.97 & & $0.47 \%$ \\
\hline \multicolumn{14}{|l|}{ Product } \\
\hline $65.0 \%$ & 250 & 162.50 & 1.29 & & & & & & $3,406.12$ & 20.96 & $6,890.74$ & 42.40 & $10.28 \%$ \\
\hline
\end{tabular}




\begin{tabular}{|c|c|c|c|c|c|c|c|c|c|c|c|c|c|}
\hline Step 5 & & & & & & & & & & & & & \\
\hline Step 4 Product & 250 & 250.00 & 1.29 & 1 & 1 & & & 20.96 & $5,240.19$ & 27.12 & $10,601.14$ & & \\
\hline Cytosine & 111 & 166.50 & 0.86 & 1.5 & 1.5 & & $14631-20-0$ & 27.00 & $4,495.50$ & 23.27 & 166.50 & & $31.91 \%$ \\
\hline Bromine & 159 & 238.50 & 1.23 & 1.5 & 1.5 & & 7726-95-6 & 2.90 & 691.65 & 3.58 & 238.50 & & $4.91 \%$ \\
\hline Mesitylene & 120.2 & 240.40 & 1.24 & 2 & 2 & 0.864 & 63148-57-2 & 3.00 & 721.20 & 3.73 & 240.40 & & $5.12 \%$ \\
\hline HMDS & 161.4 & 274.38 & 1.42 & 1.7 & 1.7 & & 10416-59-8 & 3.00 & 823.14 & 4.26 & 274.38 & & $5.84 \%$ \\
\hline Tributylamine & 185 & 277.50 & 1.44 & 1.5 & 1.5 & & $102-82-9$ & 1.40 & 388.50 & 2.01 & 277.50 & & $2.76 \%$ \\
\hline Dichloromethane & 84.93 & 662.50 & 3.43 & & $10 \mathrm{~V}$ & 1.325 & $75-09-2$ & 0.68 & 450.50 & 2.33 & 662.50 & & $3.20 \%$ \\
\hline Methanol & 32.04 & 79.10 & 0.41 & & $4 \mathrm{~V}$ & 0.791 & $67-56-1$ & 0.42 & 33.22 & 0.17 & 79.10 & & $0.24 \%$ \\
\hline Hexanes & 86.178 & 65.50 & 0.34 & & $4 \mathrm{~V}$ & 0.655 & $110-54-3$ & 1.00 & 65.50 & 0.34 & 65.50 & & $0.47 \%$ \\
\hline \multicolumn{14}{|l|}{ Product } \\
\hline $86.0 \%$ & 257 & 221.02 & 1.14 & & & & & & $12,909.40$ & 58.41 & $12,605.52$ & 57.03 & $54.45 \%$ \\
\hline \multicolumn{14}{|l|}{ Step 6} \\
\hline Step 5 Product & 257 & 257.00 & 1.14 & 1 & 1 & & & 58.41 & $15,010.93$ & 66.81 & $14,657.58$ & & \\
\hline Sodium borohydride & 37.83 & 49.18 & 0.22 & 1.3 & 1.3 & & $16940-66-2$ & 17.00 & 836.04 & 3.72 & 49.18 & & $5.10 \%$ \\
\hline EtOH & 32.04 & 203.54 & 0.91 & & $10 \mathrm{~V}$ & 0.792 & $64-17-5$ & 0.91 & 185.23 & 0.82 & 203.54 & & $1.13 \%$ \\
\hline Oxalic Acid & 90.03 & 360.12 & 1.60 & & 4 & & $144-62-77$ & 0.50 & 180.06 & 0.80 & 360.12 & & $1.10 \%$ \\
\hline 3M HCl (aq.) & 36.46 & 56.54 & 0.25 & & $2 \mathrm{~V}$ & & $7647-01-0$ & 0.75 & 42.41 & 0.19 & 56.54 & & $0.26 \%$ \\
\hline Water for $3 \mathrm{M} \mathrm{HCl}$ solution & 18.015 & 514.00 & 2.29 & & & 1 & & 0.06 & 30.84 & 0.14 & 514.00 & & $0.19 \%$ \\
\hline $45 \% \mathrm{NaOH}$ (aq.) & 40 & 194.68 & 0.87 & & $0.5 \mathrm{~V}$ & 1.515 & $1310-73-2$ & 0.48 & 93.45 & 0.42 & 194.68 & & $0.57 \%$ \\
\hline \multicolumn{14}{|l|}{ Product } \\
\hline \multirow[t]{2}{*}{$98.0 \%$} & 229.26 & 224.67 & 1.00 & & & & & & $16,378.95$ & 72.90 & $16,035.64$ & 71.37 & $8.35 \%$ \\
\hline & & & & & & & & & & $\$ / \mathbf{k g}$ & & PMI & $100.00 \%$ \\
\hline \multirow[t]{2}{*}{ Overall yield } & $34.97 \%$ & & & & & & & & & & & & \\
\hline & & & & & & & & & Total RM Cost \$ & 72.90 & & PMI & Sum of steps \\
\hline Solvent recycle & $90 \%$ & & & & & & & & & & & 71.37 & $\%$ of total \\
\hline
\end{tabular}

\section{Assumptions:}

\section{Lactic Acid: Aggressive Optimization}

o Increase solvent recycle to $90 \%$

0 Decrease reagent consumption in Step 4 (acylation)

0 Increase yield from $54 \%$ to $65 \%$ in Step 4 (acylation)

0 Reduce consumption of sodium borohydride to 1.3 equivalents in Step 6 (reduction) 


\section{Menthol Route: US8748604 (Patent)}

Table S5: Cost of Goods (CoGs) for current manufacturing route as described in US8748604.

\begin{tabular}{|c|c|c|c|c|c|c|c|c|c|c|c|c|c|c|}
\hline Raw materials 3TC & Mol. Wt. & Amount & kg RM / & Mole & \begin{tabular}{|l|} 
Equivalent \\
\end{tabular} & Density & RM Cost & RM cost & RM cost & & kg RM / & & kg RM / & \% RM Cost/ \\
\hline & & $\mathrm{kg}$ & kg Prod. & & & & $\$ / \mathrm{kg}$ & $\$ /$ batch & $\$ / \mathrm{kg}$ Prod. & kg RM & kg prod & kg RM & kg prod & \begin{tabular}{|l} 
Total RM cost \\
\end{tabular} \\
\hline \multicolumn{15}{|l|}{ Step 1} \\
\hline L-menthyl glyoxylate & 230.3 & 23.03 & 2.90 & 0.1 & 1 & & 16 & 368.48 & 46.43 & 23.03 & & 23.03 & & $27.24 \%$ \\
\hline 1,4-dithiane-2,5-diol & 152.22 & 7.61 & 0.96 & 0.05 & 0.5 & & 9.9 & 75.35 & 9.49 & 7.61 & & 7.61 & & $5.57 \%$ \\
\hline Acetic acid & 60 & 3.00 & 0.38 & 0.05 & 0.5 & 1.05 & 1.5 & 4.50 & 0.57 & 3.00 & & 3.00 & & $0.33 \%$ \\
\hline Triethylamine & 101.19 & 10.12 & 1.28 & 0.1 & 1 & 0.72 & 4.04 & 40.88 & 5.15 & 10.12 & & 10.12 & & $3.02 \%$ \\
\hline Toluene & 92.14 & 21.79 & 2.75 & & $4.35 \mathrm{~V}$ & 0.87 & 0.64 & 13.95 & 1.76 & 21.79 & & 21.79 & & $1.03 \%$ \\
\hline Heptane & 100.21 & 19.69 & 2.48 & & $5 \mathrm{~V}$ & 0.684 & 0.4 & 7.88 & 0.99 & 19.69 & & 19.69 & & $0.58 \%$ \\
\hline $\begin{array}{l}\text { Product: L-menthyl-5R-hydroxyl-1,3-oxathiolanes-2R- } \\
\text { carboxylate }\end{array}$ & 288.4 & & & & & & & & & & & & & \\
\hline $90 \%$ & 288.4 & 25.96 & 3.27 & & & & & 511.03 & 19.69 & 85.24 & 3.28 & 85.24 & 3.28 & $37.78 \%$ \\
\hline \multicolumn{15}{|l|}{ Step 2 } \\
\hline L-menthyl-5R-hydroxyl-1,3-oxathiolanes-2R-carboxylate & 288.4 & 28.84 & 3.27 & 0.1 & 1 & & 19.69 & 567.81 & & 94.71 & & 94.71 & & \\
\hline Thionyl chloride & 118.97 & 12.49 & 1.42 & 0.105 & 1.05 & 1.64 & 1.50 & 18.74 & 2.12 & 12.49 & & 12.49 & & $1.25 \%$ \\
\hline Dichloromethane(DCM) & 84.93 & 38.24 & 4.34 & & $4 \mathrm{~V}$ & 1.326 & 0.68 & 26.00 & 2.95 & 38.24 & & 38.24 & & $1.73 \%$ \\
\hline Dimethly formamide (DMF) & 73.09 & 1.84 & 0.21 & & $0.27 \mathrm{~V}$ & 0.944 & 0.95 & 1.75 & 0.20 & 1.84 & & 1.84 & & $0.12 \%$ \\
\hline Methane sulfonic acid & 96.1 & 0.14 & 0.02 & 0.002 & 0.015 & 1.48 & 6.3 & 0.91 & 0.10 & 0.14 & & 0.14 & & $0.06 \%$ \\
\hline Water & 18 & 57.68 & 6.54 & & $2 \mathrm{~V}$ & & 0.06 & 3.46 & 0.39 & 57.68 & & & & $0.23 \%$ \\
\hline $94 \%$ & 306.8 & 28.84 & 3.27 & & & & & 618.67 & 21.45 & 205.11 & 7.11 & 147.43 & 5.11 & $3.38 \%$ \\
\hline \multicolumn{15}{|l|}{ Step 3} \\
\hline L-menthyl-cis-1.3-oxathiolan-5-chloro-2-carboxylate & 306.8 & 30.68 & 3.27 & 0.1 & 1 & & 21.45 & 658.16 & & 218.20 & & 156.84 & & \\
\hline Cytosine & 111.1 & 8.78 & 0.94 & 0.079 & 0.79 & & 27 & 236.98 & 25.26 & 8.78 & & 8.78 & & $14.82 \%$ \\
\hline Ammonium sulfate & 132.14 & 0.52 & 0.05 & 0.0039 & 0.039 & 1.77 & 0.15 & 0.08 & 0.01 & 11.06 & & 0.52 & & $0.00 \%$ \\
\hline Hexamethyl disilane & 146.38 & 13.17 & 1.40 & 0.0900 & 0.9 & 0.715 & 10 & 131.74 & 14.04 & 13.17 & & 13.17 & & $8.24 \%$ \\
\hline Triethyl amine & 101.19 & 10.12 & 1.08 & & $33 \%$ & 0.726 & 4.04 & 40.90 & 4.36 & 10.12 & & 10.12 & & $2.56 \%$ \\
\hline Oxalic acid & 90.03 & 10.12 & 1.08 & & $33 \%$ & & 0.5 & 5.06 & 0.54 & 10.12 & & 10.12 & & $0.32 \%$ \\
\hline HCl(dil) & 36.46 & 0.12 & 0.01 & & $0.40 \%$ & 1.18 & 0.62 & 0.08 & 0.01 & 0.12 & & 0.12 & & $0.00 \%$ \\
\hline Dichloromethane(DCM) & 102.09 & 22.58 & 2.41 & & $2.22 \mathrm{~V}$ & 1.326 & 0.1 & 2.26 & 0.24 & 22.58 & & 22.58 & & $0.14 \%$ \\
\hline Ethyl acetate & 88.11 & 8.30 & 0.89 & & $1.2 \mathrm{~V}$ & 0.902 & 1.5 & 12.45 & 1.33 & 8.30 & & 8.30 & & $0.78 \%$ \\
\hline $\mathrm{MeOH}$ & 32.04 & 32.20 & 3.43 & & $5.3 \mathrm{~V}$ & 0.792 & 0.42 & 13.52 & 1.44 & 32.20 & & 32.20 & & $0.85 \%$ \\
\hline $\mathrm{NaCl}$ & 58.4 & 4.91 & 0.52 & & $16 \%$ & & 0.05 & 0.25 & 0.03 & 4.91 & & 4.91 & & $0.02 \%$ \\
\hline Water & 18 & 61.36 & 6.54 & & $2 \mathrm{~V}$ & & 0.06 & 3.68 & 0.39 & 61.36 & & & & $0.23 \%$ \\
\hline $77 \%$ & 381.48 & 29.37 & 3.13 & & & & & $1,105.16$ & 37.62 & 400.92 & 13.65 & 267.66 & 9.11 & $27.95 \%$ \\
\hline
\end{tabular}




\begin{tabular}{|c|c|c|c|c|c|c|c|c|c|c|c|c|c|c|}
\hline Step 4 & & & & & & & & & & & & & & \\
\hline $\begin{array}{l}\text { L-menthyl 1,3-oxathiolan-5S-(cytosin-1-yl)-2R-carboxylate } \\
\text { oxalate }\end{array}$ & 381.48 & 38.15 & 3.13 & 0.1 & 1 & & 37.62 & $1,435.27$ & & 520.68 & & 347.61 & & \\
\hline Dipotassium hydrogen phosphate & 174.2 & 52.26 & 4.29 & 0.3 & 3 & & 1.5 & 78.39 & 6.43 & 52.26 & & 52.26 & & $3.77 \%$ \\
\hline Sodium Borohydride & 37.83 & 6.39 & 0.52 & 0.169 & 1.69 & & 26 & 166.23 & 13.64 & 6.39 & & 6.39 & & $8.00 \%$ \\
\hline Sodium Hydroxide & 40 & 11.44 & 0.94 & & $30 \%$ & & 0.48 & 5.49 & 0.45 & 11.44 & & 11.44 & & $0.26 \%$ \\
\hline $\mathrm{HCl}$ & 36.5 & 0.20 & 0.16 & & $5.20 \%$ & 1.18 & 0.1 & 0.02 & 0.02 & 0.20 & & 0.20 & & $0.01 \%$ \\
\hline Dichloromethane(DCM) & 84.93 & 126.46 & 10.38 & & $10 \mathrm{~V}$ & 1.326 & 0.68 & 85.99 & 7.06 & 126.46 & & 126.46 & & $4.14 \%$ \\
\hline Ethanol & 46.07 & 75.25 & 6.18 & & $10 \mathrm{~V}$ & 0.789 & 0.91 & 68.47 & 5.62 & 75.25 & & 75.25 & & $3.30 \%$ \\
\hline Isopropanol & 60.1 & 55.47 & 4.55 & & $7.4 \mathrm{~V}$ & 0.786 & 1.43 & 79.32 & 6.51 & 55.47 & & 55.47 & & $3.82 \%$ \\
\hline Carbon & 12 & 1.91 & 0.16 & & $5 \%$ & & 10.75 & 20.50 & 1.68 & 1.91 & & 1.91 & & $0.99 \%$ \\
\hline water & 18 & 419.63 & 34.44 & & $11 \mathrm{~V}$ & 1 & 0.06 & 25.18 & 2.07 & 419.63 & & & & $1.21 \%$ \\
\hline $73 \%$ & 229.26 & 16.74 & 1.37 & & & & & $1,964.87$ & 117.40 & $1,269.69$ & 75.87 & 676.99 & 40.45 & $25.51 \%$ \\
\hline \multicolumn{15}{|l|}{ Step 5} \\
\hline $\begin{array}{l}\text { 4-amino-1-[(2R,5S)-2-(hydroxymethyl)1,3-oxathiolan-5-yl]-1,2- } \\
\text { dihydropyrimidin-2-one (Crude 3TC.HCI) }\end{array}$ & 229.26 & 22.93 & 1.37 & 0.1 & 1 & & 117.40 & $2,691.60$ & & $1,739.30$ & & 927.39 & & \\
\hline Tribuyl amine(TBA) & 185.36 & 21.50 & 1.29 & 0.116 & 1.16 & & 1.43 & 30.75 & 1.84 & 21.50 & & 21.50 & & $1.08 \%$ \\
\hline Dichloromethane(DCM) & 84.93 & 56.24 & 3.37 & & $7.4 \mathrm{~V}$ & 1.326 & 0.68 & 38.24 & 2.29 & 56.24 & & 56.24 & & $1.34 \%$ \\
\hline $91 \%$ & 229.26 & 20.86 & 1.25 & & & & & $2,760.59$ & 132.32 & $1,817.04$ & 87.10 & $1,005.13$ & 48.18 & $2.43 \%$ \\
\hline \multicolumn{15}{|l|}{ Step 6} \\
\hline $\begin{array}{l}\text { 4-amino-1-[(2R,5S)-2-(hydroxymethyl)1,3-oxathiolan-5-yl]-1,2- } \\
\text { dihydropyrimidin-2-one (Crude 3TC) }\end{array}$ & 229.26 & 22.93 & 1.25 & 0.1 & 1 & & 132.32 & $3,033.62$ & & $1,996.75$ & & $1,104.54$ & & \\
\hline Carbon & 12 & 1.28 & 0.07 & & $5.60 \%$ & & 1.26 & 1.62 & 0.09 & 1.28 & & 1.28 & & $0.05 \%$ \\
\hline Hyflo & & 6.05 & 0.33 & & $26.40 \%$ & & 2.20 & 13.32 & 0.73 & 6.05 & & 6.05 & & $0.43 \%$ \\
\hline Isopropanol & 60.1 & 54.06 & 2.95 & & $12 \mathrm{~V}$ & 0.786 & 1.43 & 77.31 & 4.22 & 54.06 & & 54.06 & & $2.47 \%$ \\
\hline \multicolumn{15}{|l|}{$\begin{array}{l}\text { 4-amino-1-[(2R,5S)-2-(hydroxymethyl)1,3-oxathiolan-5-yl]-1,2- } \\
\text { dihydropyrimidin-2-one (Pure 3TC) }\end{array}$} \\
\hline $80 \%$ & 229.24 & 18.34 & 1.00 & & & & & $3,125.85$ & 170.45 & $2,058.14$ & 112.23 & $1,165.93$ & 63.58 & $2.95 \%$ \\
\hline & & & & & & & \multicolumn{2}{|c|}{1 mole KRM case } & $\$ / \mathrm{kg} 3 \mathrm{TC}$ & & PMI & & PMI & $100.00 \%$ \\
\hline \multirow[t]{2}{*}{ Overall Yield } & $34.62 \%$ & & & & & & & & & \multicolumn{2}{|r|}{ with water } & \multicolumn{2}{|r|}{ w/o water } & \\
\hline & & & & & & & & & & & & & & Sum of steps \\
\hline \multirow[t]{4}{*}{ Solvent recycle } & $75 \%$ & & & & & & & & & & & & & $\%$ of total \\
\hline & & & & & & & \multicolumn{2}{|c|}{$1 \mathrm{~kg} 3 \mathrm{TC}$ case } & 170.46 & & 111.25 & & 63.72 & $100.00 \%$ \\
\hline & & & & & & & & & $\$ / \mathrm{kg} 3 \mathrm{TC}$ & & PMI & & PMI & \\
\hline & & & & & & & & & & & with water & & w/o water & \\
\hline
\end{tabular}




\section{Ozonolysis to form 1:}

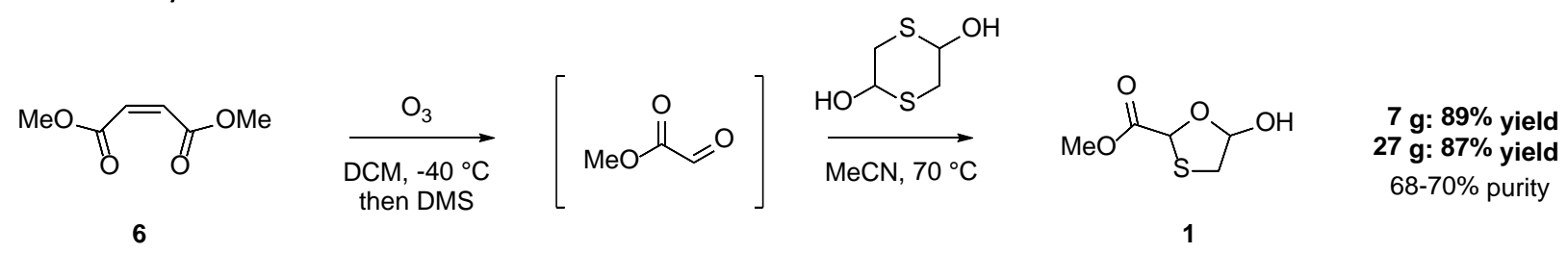

Table S6: Ozonolysis of dimethyl maleate.

\begin{tabular}{ccccc} 
Scale & $\begin{array}{c}\text { Ozonolysis } \\
\text { Solvent }\end{array}$ & Product (g) & Purity & Adjusted Yield \\
\hline $0.500 \mathrm{~g}$ & MeCN & 1.26 & 66 & $77 \%$ \\
$0.500 \mathrm{~g}$ & $\mathrm{DCM}$ & 1.35 & 68 & $84 \%$ \\
$2.50 \mathrm{~g}$ & $\mathrm{DCM}$ & 7.09 & 69 & $89 \%$ \\
$10.6 \mathrm{~g}$ & $\mathrm{MeCN}$ & 26.4 & 63 & $72 \%$ \\
$10.0 \mathrm{~g}$ & $\mathrm{DCM}$ & 27.3 & 70 & $87 \%$ \\
\hline
\end{tabular}

\section{Typical procedure for synthesis of methyl 5-hydroxy-1,3-oxathiolane-2-carboxylate (1):}

Dimethyl maleate (10.0 g, $66.6 \mathrm{mmol}, 1$ equiv., 96\% Sigma-Aldrich) was added into a two-neck round bottom flask fitted with an Ozone inlet and outlet. The outlet was connected to a scrubber filled with saturated sodium thiosulfate solution to quench the ozone. Dichloromethane $(90 \mathrm{~mL}, 99.8 \%$, MilliporeSigma) was charged into the vessel. The solution was cooled to $-40{ }^{\circ} \mathrm{C}$, and the ozone generator was turned on. Oxygen feed was turned on at a rate of $1.5-2.5 \mathrm{~L} / \mathrm{min}$. The ozone was bubbled through solution, and the gas flow was kept to a minimum in order to prevent loss of either dichloromethane or methyl glyoxylate from the system. Once the solution turned a light blue indicating saturation with ozone, the reaction was monitored by NMR for consumption of dimethyl maleate. When less than $5 \%$ of dimethyl maleate remained (typically $1 \mathrm{hr}$ ), the ozonolysis unit was turned off and oxygen flow was stopped. The solution was sparged with nitrogen to remove presence of ozone. While still cooled, dimethyl sulfide ( $6.40 \mathrm{~mL}, 86.6 \mathrm{mmol}, 1.3$ equiv., $>99 \%$ Sigma-Aldrich) was added to quench ozonide and form the methyl glyoxylate. The cold solution was stirred for $3 \mathrm{hr}$ at $-40{ }^{\circ} \mathrm{C}$, removed from the cooling bath and allowed to warm slowly to room temperature. Once at room temperature, the reaction stirred for 6 hours prior to addition of 1,4-dithiane-2,5-diol ( $10.1 \mathrm{~g}, 66.6 \mathrm{mmol}, 1$ equiv., $96 \%$ Alfa-Aesar). Acetonitrile $(50 \mathrm{~mL},>95 \%$ MilliporeSigma) was added and the reaction was heated to an internal temperature of $70{ }^{\circ} \mathrm{C}$, driving off the dichloromethane and dimethyl sulfide. The reaction was kept at $70{ }^{\circ} \mathrm{C}$ for 8 hours. Solvent and volatiles were removed under vacuum to give a light yellow to orange oil. Toluene was added and the mixture was heated at $50{ }^{\circ} \mathrm{C}$ under vacuum to remove any water from the system. $\quad 27.3 \mathrm{~g}$ of $1 \mathbf{1 7 0 . 0 \%}$ purity) was obtained ( $116 \mathrm{mmol}, 87.4 \%)$. The remainder of the material was related to dimethyl sulfoxide and what is believed to be oligomers/polymer of dimethyl glyoxylate. The material can be used directly in the subsequent step or can be purified by extraction as described below. ${ }^{1} \mathrm{H} \mathrm{NMR}\left(600 \mathrm{MHz}, \mathrm{CDCl}_{3}\right) \delta$ $6.05-5.85(1 \mathrm{H}), 5.59(1 \mathrm{H}), 4.77-4.30(1 \mathrm{H}), 3.78(3 \mathrm{H}), 3.29-3.20(1 \mathrm{H}), 3.14-2.99(1 \mathrm{H}) .{ }^{13} \mathrm{C} \mathrm{NMR}(151$ $\mathrm{MHz}, \mathrm{CDCl}_{3}$ ) $\delta 172.59$ (s), 170.48 (s), 102.66 (s), 101.22 (s), 79.31 (s), 77.49 (s), 53.23 (s), 52.79 (s), 39.83 (s), 38.29 (s). ${ }^{1} \mathrm{H}$ NMR (600 MHz, DMSO) $\delta 7.08-7.00(\mathrm{~m}, 1 \mathrm{H}), 5.87(\mathrm{~m}, 1 \mathrm{H}), 5.61(\mathrm{~s}, 1 \mathrm{H}), 3.74-3.63(\mathrm{~s}$, 
3H), $3.17-3.08(\mathrm{~m}, 1 \mathrm{H}), 2.87(\mathrm{~m}, 1 \mathrm{H}) .{ }^{13} \mathrm{C}$ NMR (151 MHz, DMSO) $\delta$ 170.63, 170.47, 102.08, 101.40, 76.76), 76.45, 52.70, 38.12. HRMS: Expected M-H m/z 163.0065, Found: 163.0080.

\section{Safety Notes:}

Ozonolysis reactions are very hazardous due to presence of highly reactive species (oxygen, ozone, ozonide, and hydroperoxides which may form in the quench). A blast shield should always be in place as a precautionary measure. The quench of the ozonide with sulfide is exothermic and should be conducted at low temperature. The reaction should not be cooled with liquid nitrogen to avoid condensation of liquid oxygen. After finishing the reaction, the mixture should be sparged with nitrogen rather than oxygen to remove ozone, but also to eliminate hazards presented in having a highly oxygenated headspace during the dimethyl sulfide quench.

\section{Reaction Sensitivities-Important Notes for Reproduction:}

- If oxygen is passed through the system at too high a relative rate, then volatile solvent and glyoxylate can be lost thus reducing yield

- Monitor reaction for complete conversion of dimethyl maleate during ozonolysis. Reaction will turn blue indicating saturation with ozone prior to full consumption of starting material.

- Dithianediol should be added before solvent swap and heating to distill dichloromethane in order to prevent loss of volatile methyl glyoxylate and to trap aldehyde prior to oligomerization of starting material

- During heating to form oxathiolane internal temperature of solution should be used rather than temperature of heating medium. Reflux/boiling of dichloromethane will cool internal solution, achieving required reaction temperature will be difficult, and dichloromethane will not be sufficiently removed. 
Optional Purification by extraction:

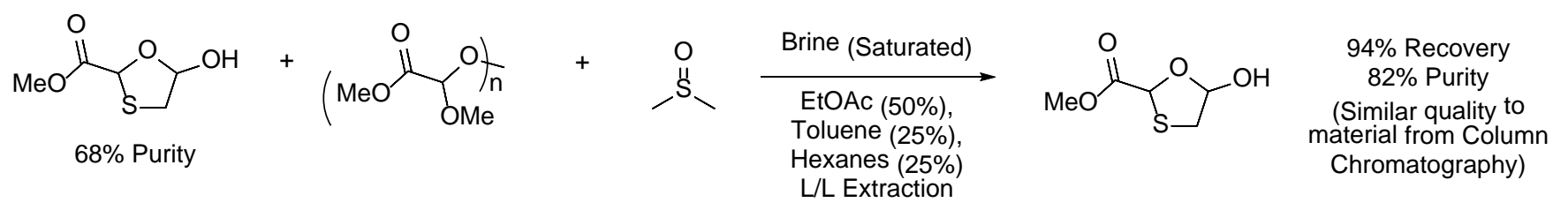

Figure S1: Oxathiolane synthesis with extractive purification performed at $5 \mathrm{~g}$ scale.

The $6.757 \mathrm{~g}$ of oxathiolane (69\% purity) was taken up in $40 \mathrm{~mL}$ of an ethyl acetate, toluene, hexanes mixture (2:1:1) and transferred to an extraction funnel. A saturated solution of $\mathrm{NaCl}$ in water $(10 \mathrm{~mL})$ was added. The solution was shaken vigorously and the layers were allowed to partition. The organic layer was collected, and the aqueous layer was gently rinsed twice with $2 \mathrm{~mL}$ of the organic solvent mixture. The organic fractions were combined and concentrated. Toluene was added and the mixture was concentrated again, at $50{ }^{\circ} \mathrm{C}$ to remove any residual water. $5.346 \mathrm{~g}$ (83\% purity) of oxathiolane was recovered (95\%).

Table S7: Initial Screening Results For Extraction Using Low-Grade Starting Material of 50\% purity:

\begin{tabular}{|c|c|c|c|c|c|c|c|c|c|c|}
\hline Solvent & $\begin{array}{l}\text { Relative } \\
\text { Volumes }\end{array}$ & Antisolvent & $\begin{array}{l}\text { Relative } \\
\text { Volumes }\end{array}$ & Sample (g) & $\begin{array}{c}\text { \# of } \\
\text { Extractions }\end{array}$ & $\begin{array}{c}\text { Amt } \\
\text { Recovered (g) }\end{array}$ & Purity & Recovery & $\begin{array}{l}\text { Polymer } \\
\text { (equiv.) }\end{array}$ & $\begin{array}{c}\text { DMSO } \\
\text { (equiv.) }\end{array}$ \\
\hline None-Material to be Purified & - & - & - & 0.446 & 0 & - & $50.0 \%$ & NA & 0.09 & 0.23 \\
\hline EtOAc & 6 & Water & 1.5 & 0.446 & 1 & NA-Miscible & NA & NA & NA & NA \\
\hline EtOAc & 6 & Brine & 1.5 & 0.446 & 6 & 0.305 & $62.0 \%$ & $84.8 \%$ & Little & 0 \\
\hline THF & 6 & Brine & 1.5 & 0.446 & 6 & 0.372 & $48.0 \%$ & $80.1 \%$ & Significant & 0 \\
\hline EtOAc & 6 & Brine & 1.5 & 0.446 & 1 & 0.383 & $58.0 \%$ & $99.6 \%$ & 0.08 & 0.06 \\
\hline EtOAc(50\%)/Toluene(50\%) & 6 & Brine & 1.5 & 0.446 & 1 & 0.357 & $62.0 \%$ & $99.3 \%$ & 0.06 & 0.05 \\
\hline EtOAc(50\%)/Toluene(25\%)/Hexanes(25\%) & 6 & Brine & 1.5 & 0.396 & 1 & 0.314 & $62.0 \%$ & $98.3 \%$ & 0.035 & 0.05 \\
\hline EtOAc & 6 & Brine & 1.5 & 0.446 & 6 & 0.283 & $65.0 \%$ & $82.5 \%$ & 0.06 & 0.002 \\
\hline EtOAc(50\%)/Toluene(50\%) & 6 & Brine & 1.5 & 0.446 & 6 & 0.2765 & $64.0 \%$ & $79.4 \%$ & 0.023 & 0.002 \\
\hline EtOAc(50\%)/Hexanes(50\%) & 6 & Brine & 1.5 & 0.446 & 6 & 0.251 & $65.0 \%$ & $73.2 \%$ & 0 & 0 \\
\hline DCM(50\%)/Toluene(50\%) & 6 & Brine & 1.5 & 0.446 & 6 & 0.311 & $62.0 \%$ & $86.5 \%$ & 0 & 0.025 \\
\hline
\end{tabular}



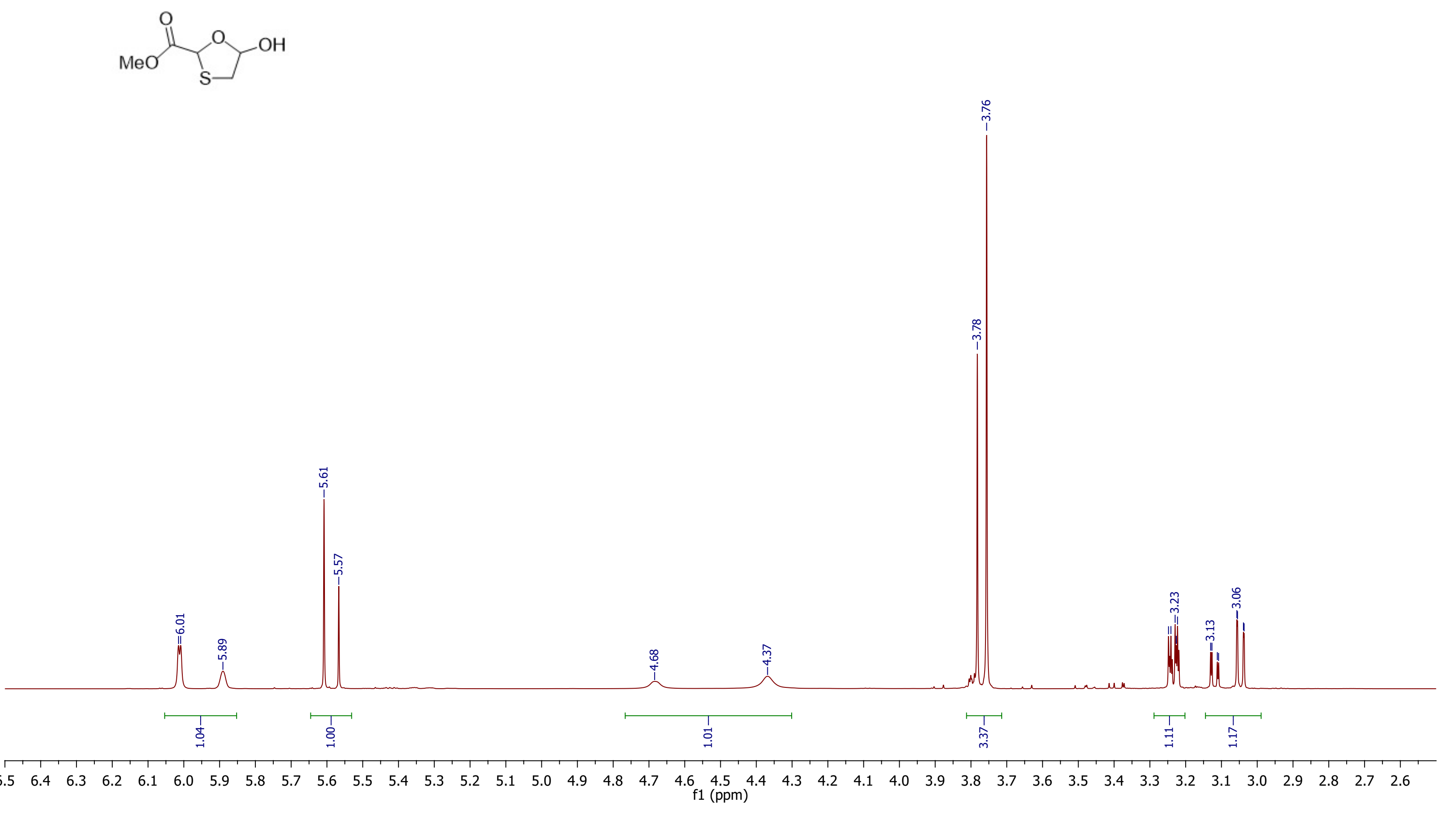
<smiles>COC(=O)C1OC(O)CS1</smiles>
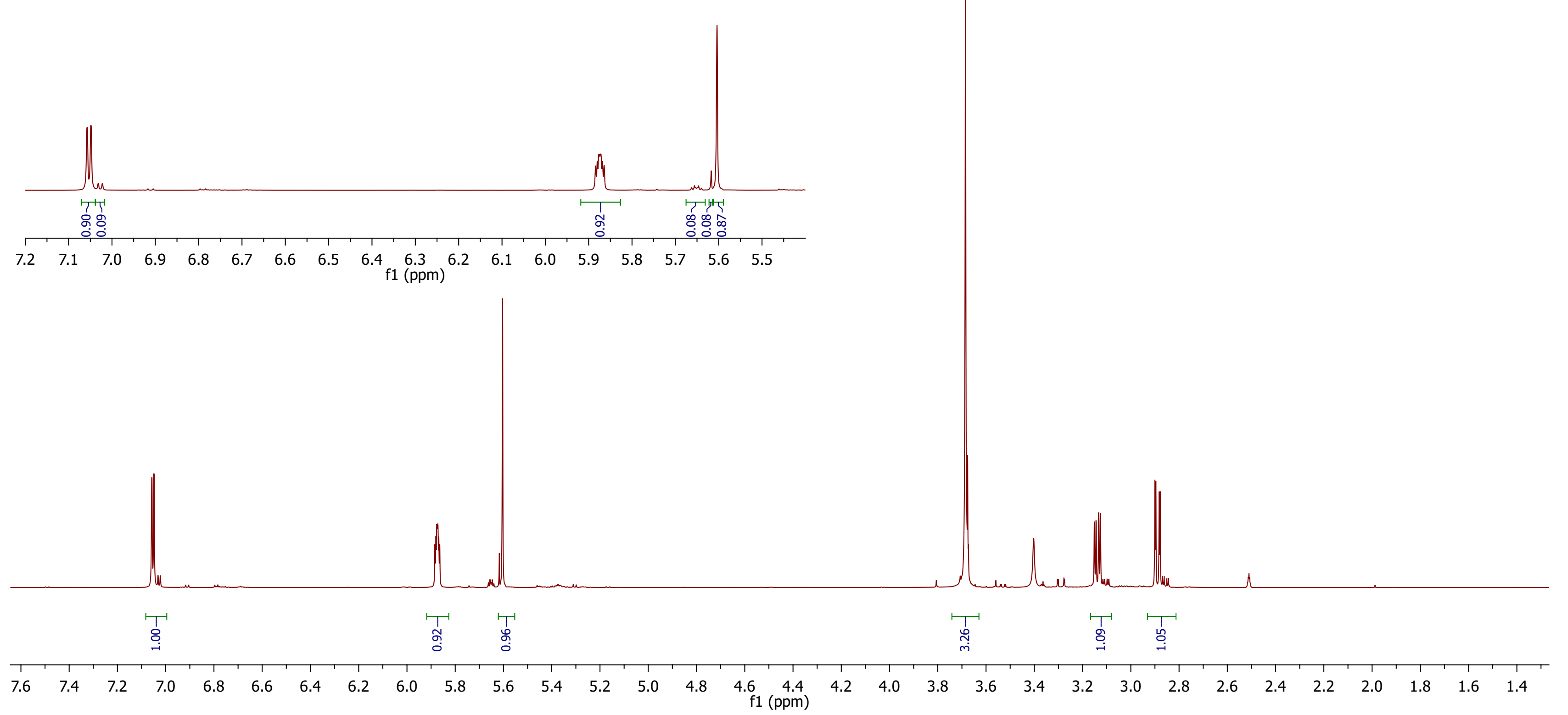
methyl 5-hydroxy-1,3-oxathiolane-2-carboxylate<smiles>COC(=O)C1OC(O)CS1</smiles>

${ }^{13} \mathrm{CNMR}\left(151 \mathrm{MHz}, \mathrm{CDCl}_{3}\right) \delta 172.59$ (s), 170.48 (s), 102.66 (s),

$101.22(\mathrm{~s}), 79.31(\mathrm{~s}), 77.49(\mathrm{~s}), 53.23(\mathrm{~s}), 52.79(\mathrm{~s}), 39.83(\mathrm{~s}), 38.29(\mathrm{~s})$.

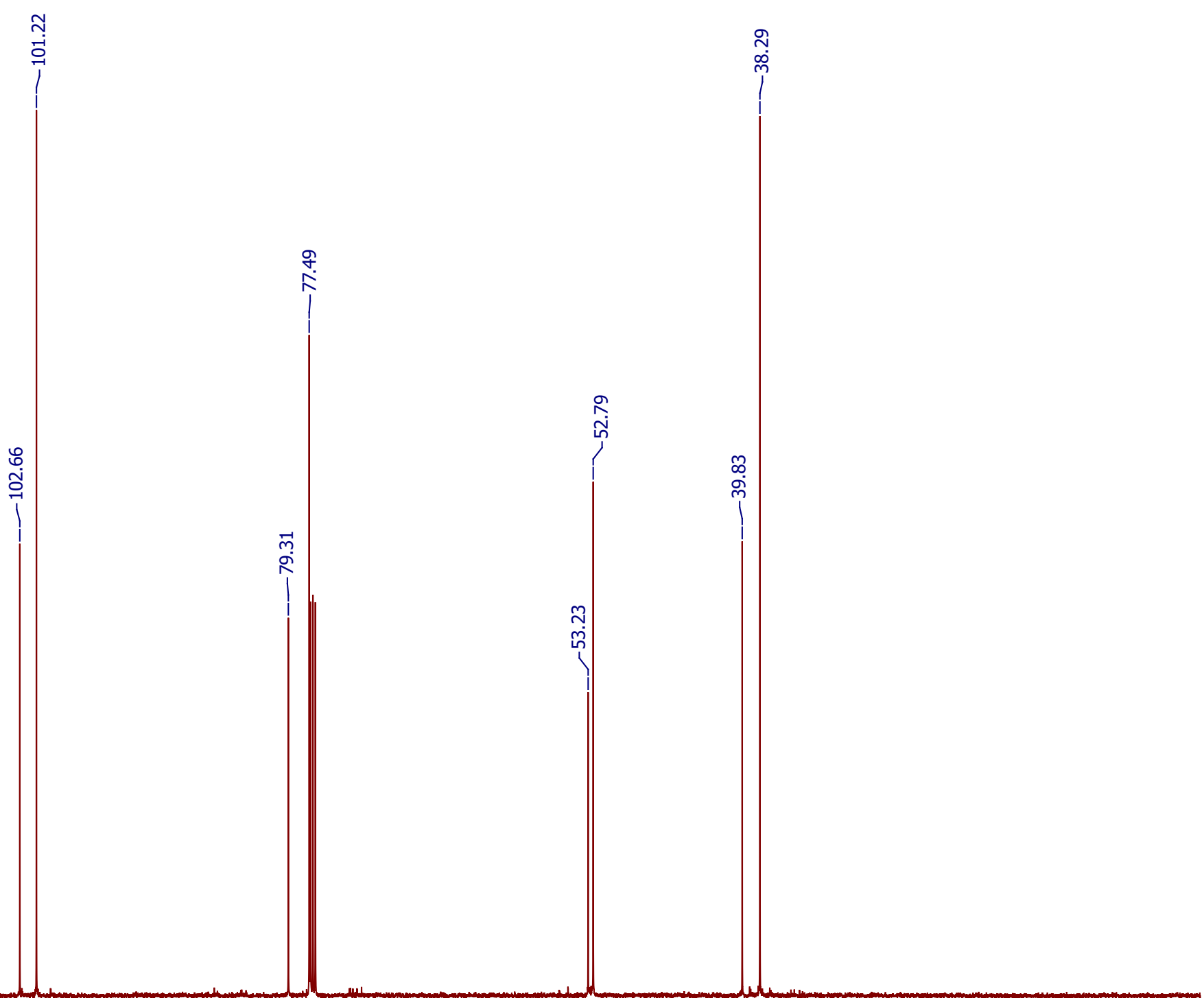


<smiles>COC(=O)C1OC(O)CS1</smiles>
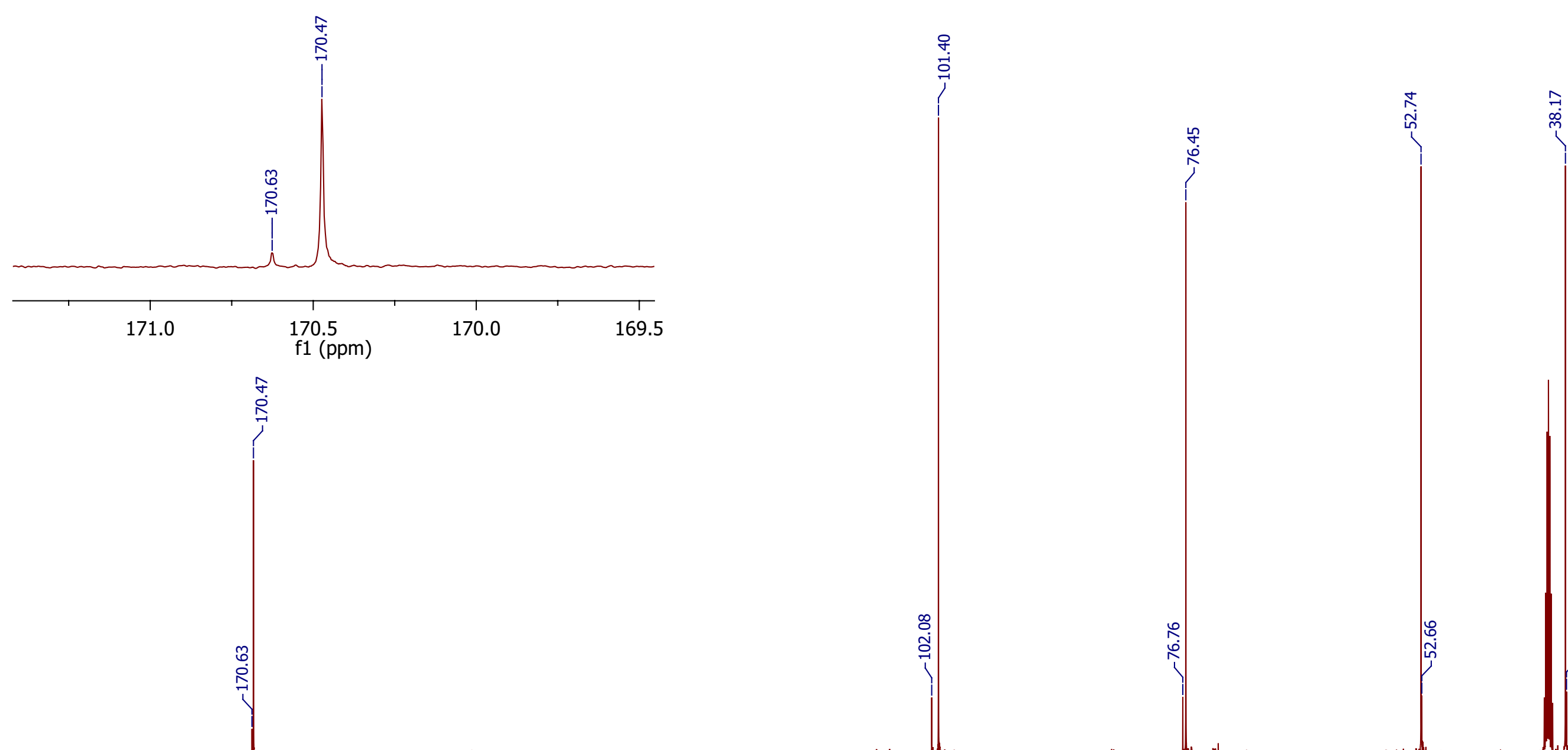
Ozonolysis Sample Assayed for Purity by

Quantitative NMR vs Mesitylene: $70 \%$

Yield adjusted for Purity: $87 \%$ (27.3 g)

DMSO: $28 \mathrm{~mol} \%$

Oligomer/Polymer: 15 mol\%

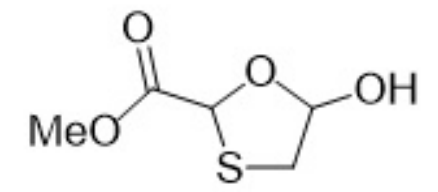

\section{Mesitylene}

\section{Mesitylene}

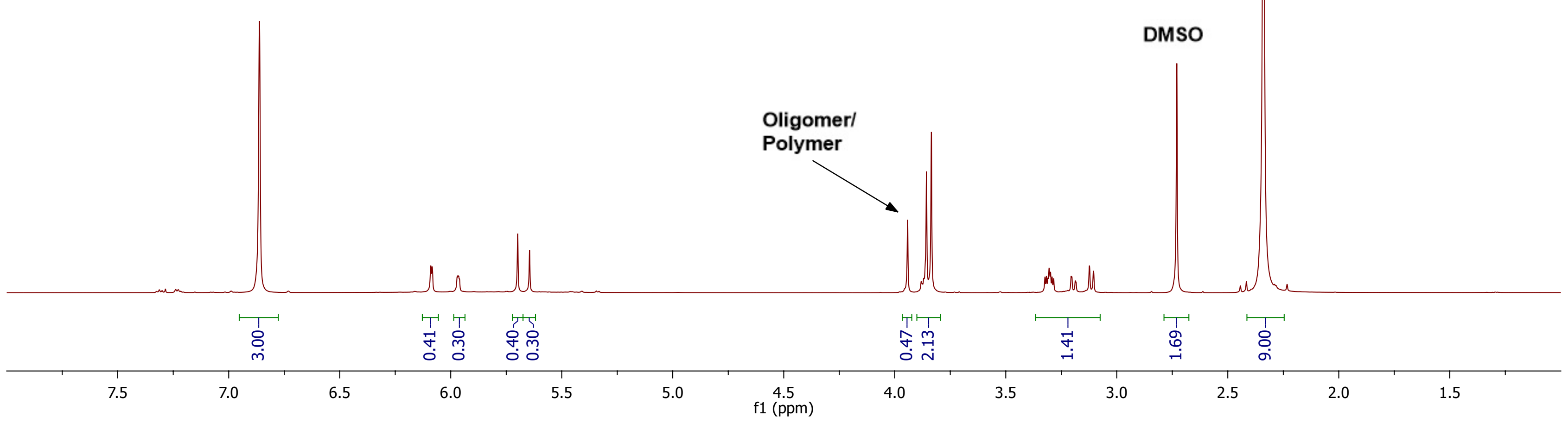


Sample Assayed for Purity after 1 Extraction:

Quantitative NMR vs Mesitylene: $83 \%$

Recovery: $95 \%$

DMSO: $7 \mathrm{~mol} \%$

Oligomer/Polymer: $1 \mathrm{~mol} \%$

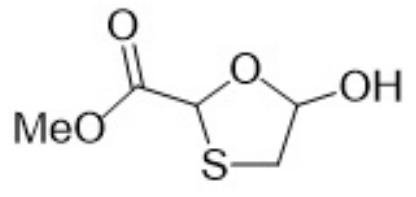

Mesitylene

Mesitylene

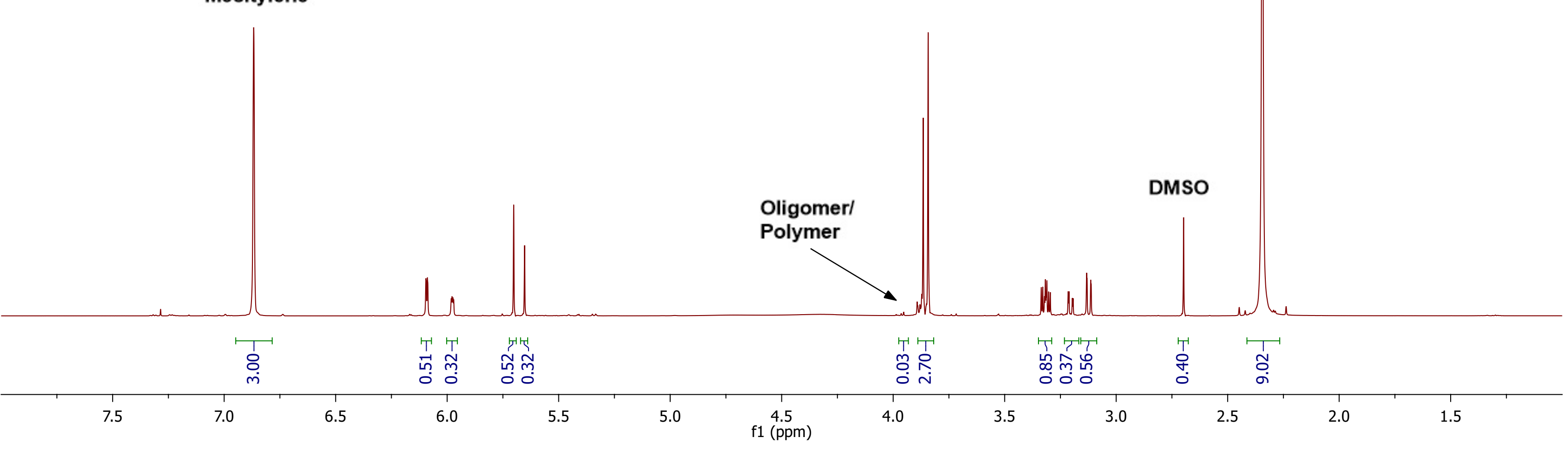




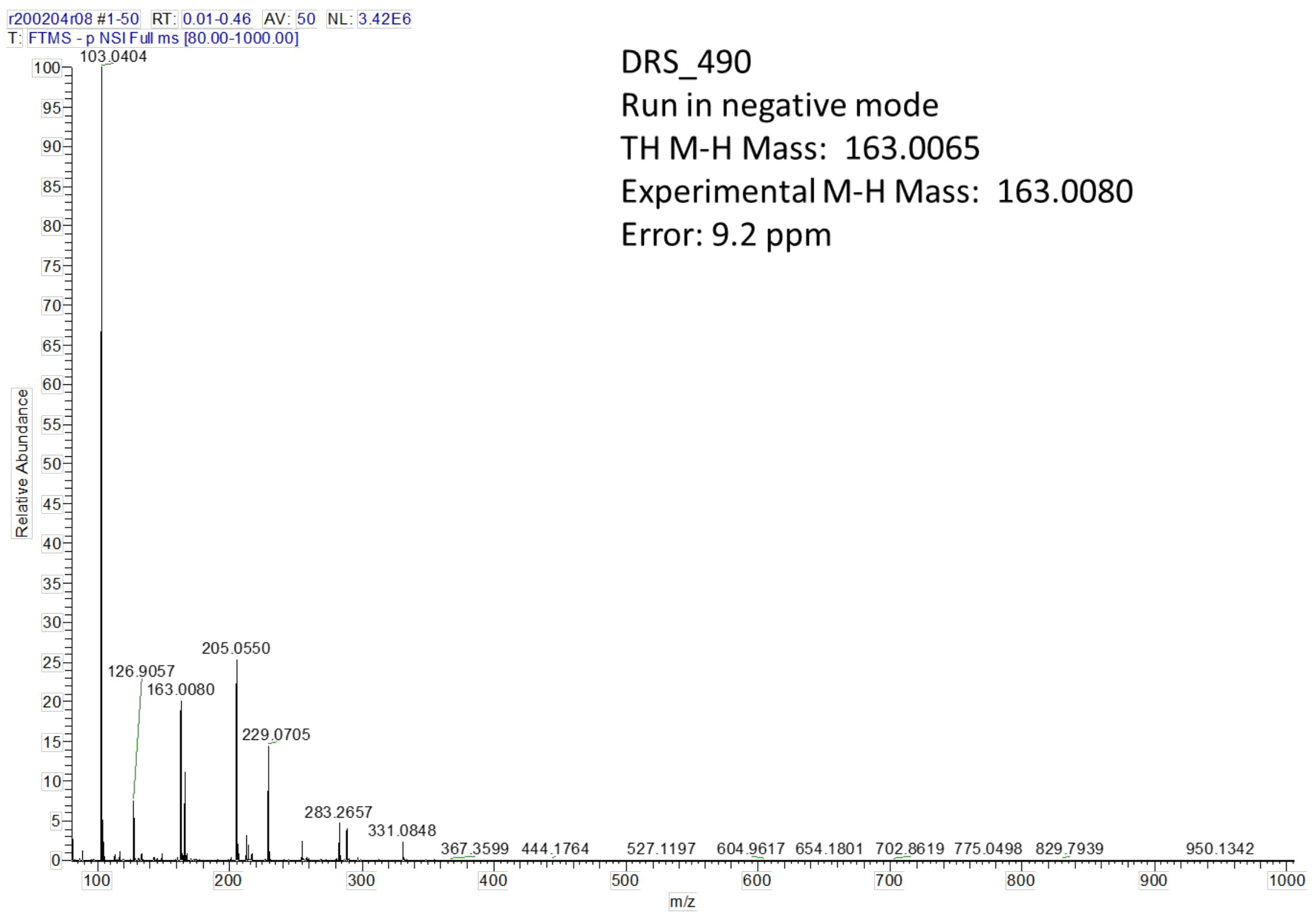


Methoxylation to form 5:<smiles>CC(Cl)C(=O)O</smiles>

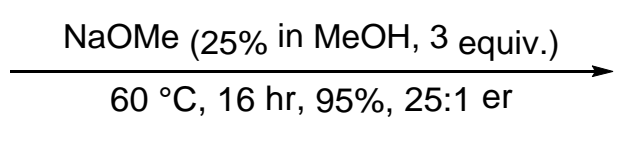<smiles>COC(=O)C(C)OC</smiles>

Table S8: Etherification of 2-chloropropanoic acid.

\begin{tabular}{ccccc} 
Entry & Scale & Product $(\mathbf{g})$ & Purity & Adjusted Yield \\
\hline 1 & $5.00 \mathrm{~g}$ & 5.70 & $96 \%$ & $94 \%$ \\
2 & $20.0 \mathrm{~g}$ & 23.7 & $91.3 \%$ & $93.1 \%$ \\
3 & $20.0 \mathrm{~g}$ & 23.5 & $94 \%$ & $95 \%$ \\
4 & $40.0 \mathrm{~g}$ & 48.3 & $91 \%$ & $95 \%$ \\
\hline
\end{tabular}

\section{Procedure for Synthesis of Sodium (R)-2-methoxypropanoate (5):}

(S)-2-Choropropanoic acid (40.0 g, 369 mmol, 1 equiv., 98\% Combi-Blocks) was added to a two-neck round-bottom flask under nitrogen. $25 \mathrm{wt} \%$ Sodium methoxide in methanol $(253 \mathrm{~mL}, 1.106 \mathrm{~mol}, 3$ equiv., Sigma-Aldrich) was added slowly. The reaction was heated to $60{ }^{\circ} \mathrm{C}$ for 16 hours and conversion was monitored until $<2 \%$ starting material remained. When sufficient conversion was achieved, the reaction vessel was cooled to room temperature, and $\mathrm{pH}$ was adjusted with $4 \mathrm{M} \mathrm{HCl}$ in dioxane $(96 \mathrm{~mL}$, 99\% Alfa-Aesar) to the point at which $\mathrm{pH}$ just changes from $>12$ to 7 , indicating neutralization of excess sodium methoxide without protonating the sodium salt of the carboxylate. The reaction mixture was filtered to remove salts, and the salt cake was washed twice with $5 \mathrm{~mL}$ of methanol. The filtrate was concentrated, and then $10 \mathrm{~mL}$ of toluene was added. Obtaining anhydrous material is important for the subsequent acylation of hydroxyoxathiolane, and thus the toluene was removed at $50{ }^{\circ} \mathrm{C}$ under vacuum to azeotropically remove water and obtain the product as a crisp white solid. Toluene was added to product and evaporated three times ( $30 \mathrm{~mL}$ total). $48.3 \mathrm{~g}$ of 5 was obtained in $91 \%$ purity ( $95 \%$ yield). ${ }^{1} \mathrm{H}$ NMR (600 MHz, D $2 \mathrm{O} \_$salt) $\delta 3.65$ (q, $\left.J=6.8 \mathrm{~Hz}, 1 \mathrm{H}\right), 3.21(\mathrm{~s}, 3 \mathrm{H}), 1.20$ (d, J=6.9 Hz, 3H). ${ }^{13} \mathrm{C} \mathrm{NMR} \mathrm{(151}$ $\mathrm{MHz}, \mathrm{D}_{2} \mathrm{O} \_$salt) $\delta 181.41,78.68,56.54,18.21$. HRMS: Expected M-H m/z 103.0395, Found: 103.0404.

Procedure for checking optical purity-synthesis of the menthyl ester:<smiles>COC(=O)C(C)O[Na]</smiles>
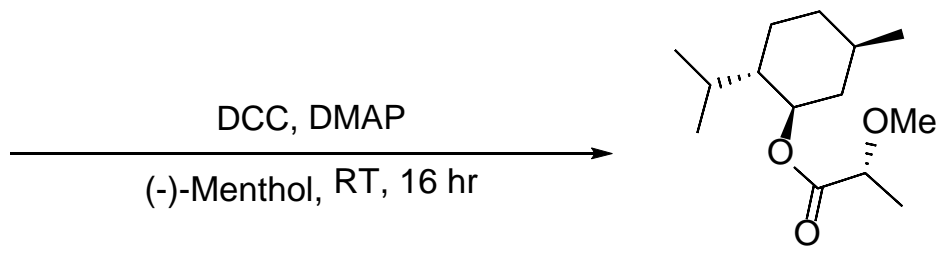

Figure S2: Synthesis of menthyl ester to assess optical purity.

Sodium (R)-2-methoxypropanoate $(70.0 \mathrm{mg}, 0.555 \mathrm{mmol})$, dicyclohexylmethanediimine (DCC, $183 \mathrm{mg}$, $0.888 \mathrm{mmol}$ ), N,N-dimethylaminopyridine (DMAP, $136 \mathrm{mg}, 1.11 \mathrm{mmol})$, and (-)-menthol (217 mg, 1.39 
$\mathrm{mmol}$ ) were combined in a round bottom flask under air. Dichloromethane $(3 \mathrm{~mL})$ was added. The reaction was stirred overnight. Dichloromethane was evaporated, and the residuals were dry-loaded onto silica gel. The ester was purified by flash column chromatography (10:1 Hexanes/EtOAc). $107 \mathrm{mg}$ of the menthol ester was recovered $(80 \%)$. The signal for the methyl ether was used to assess optical purity by comparing to material made from (R/S)-2-methoxypropanoate. ${ }^{1} \mathrm{H} N M R\left(600 \mathrm{MHz}, \mathrm{CDCl}_{3}\right) \delta$ 4.70 (ddd, $J=21.8,10.9,4.4 \mathrm{~Hz}, 1 \mathrm{H}$ ), $3.77(\mathrm{qd}, J=6.8,4.1 \mathrm{~Hz}, 1 \mathrm{H}), 3.31(\mathrm{~d}, J=9.9 \mathrm{~Hz}, 3 \mathrm{H}), 2.00-1.87(\mathrm{~m}$, $1 \mathrm{H}), 1.87-1.75(\mathrm{~m}, 1 \mathrm{H}), 1.62(\mathrm{~m}, 2 \mathrm{H}), 1.50-1.40(\mathrm{~m}, 1 \mathrm{H}), 1.40-1.29(\mathrm{~m}, 4 \mathrm{H}), 1.05-0.89(\mathrm{~m}, 2 \mathrm{H}), 0.86$ $-0.75(\mathrm{~m}, 7 \mathrm{H}), 0.70(\mathrm{~m}, 3 \mathrm{H}) .{ }^{13} \mathrm{C} \mathrm{NMR}\left(151 \mathrm{MHz}, \mathrm{CDCl}_{3}\right) \delta 172.71,76.59,74.80,74.55,57.56,57.41$, $46.86,40.76,34.17,31.34,26.29,26.06,23.36,23.06,21.94,20.70,18.45,16.17,15.81$. 
<smiles></smiles>

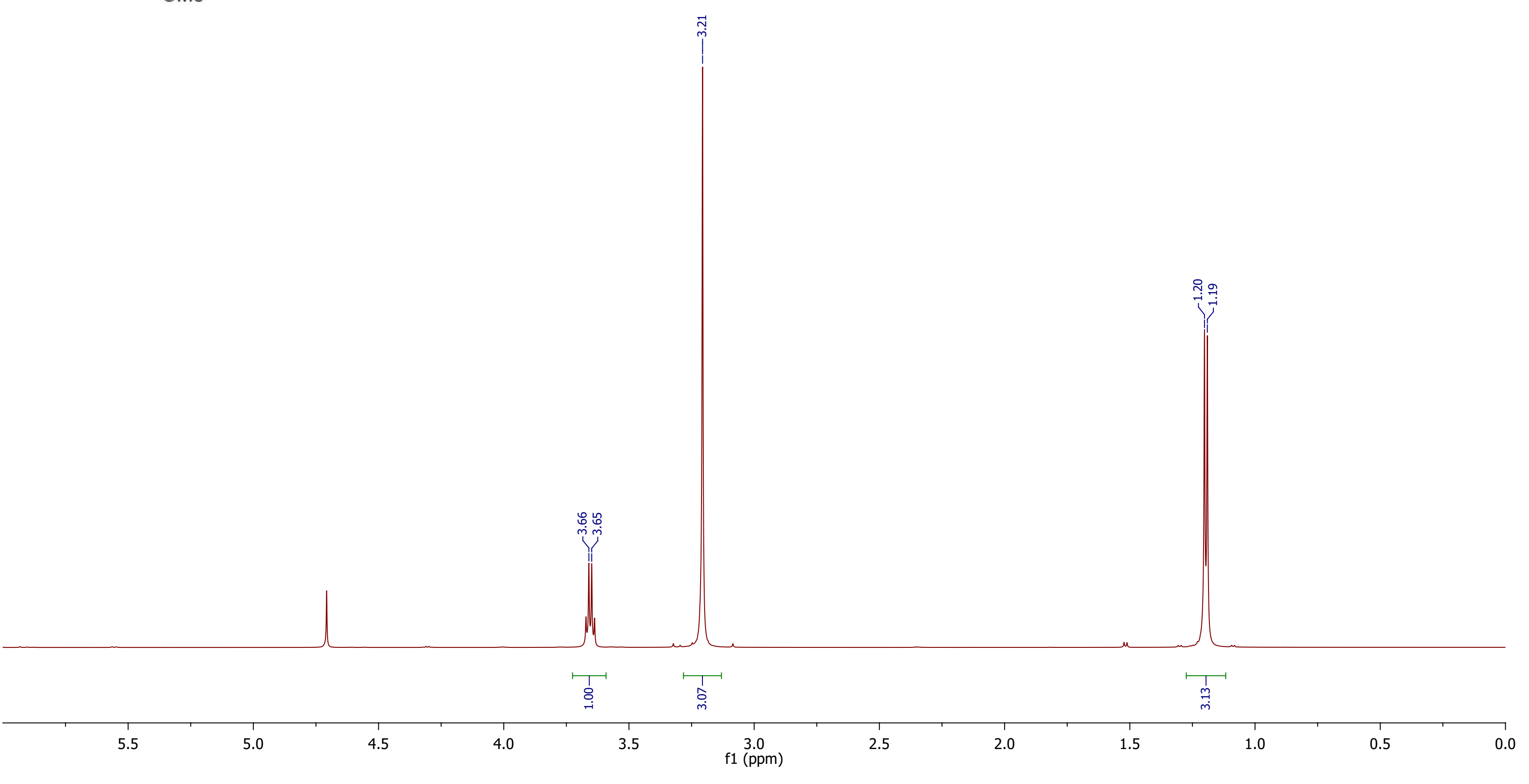



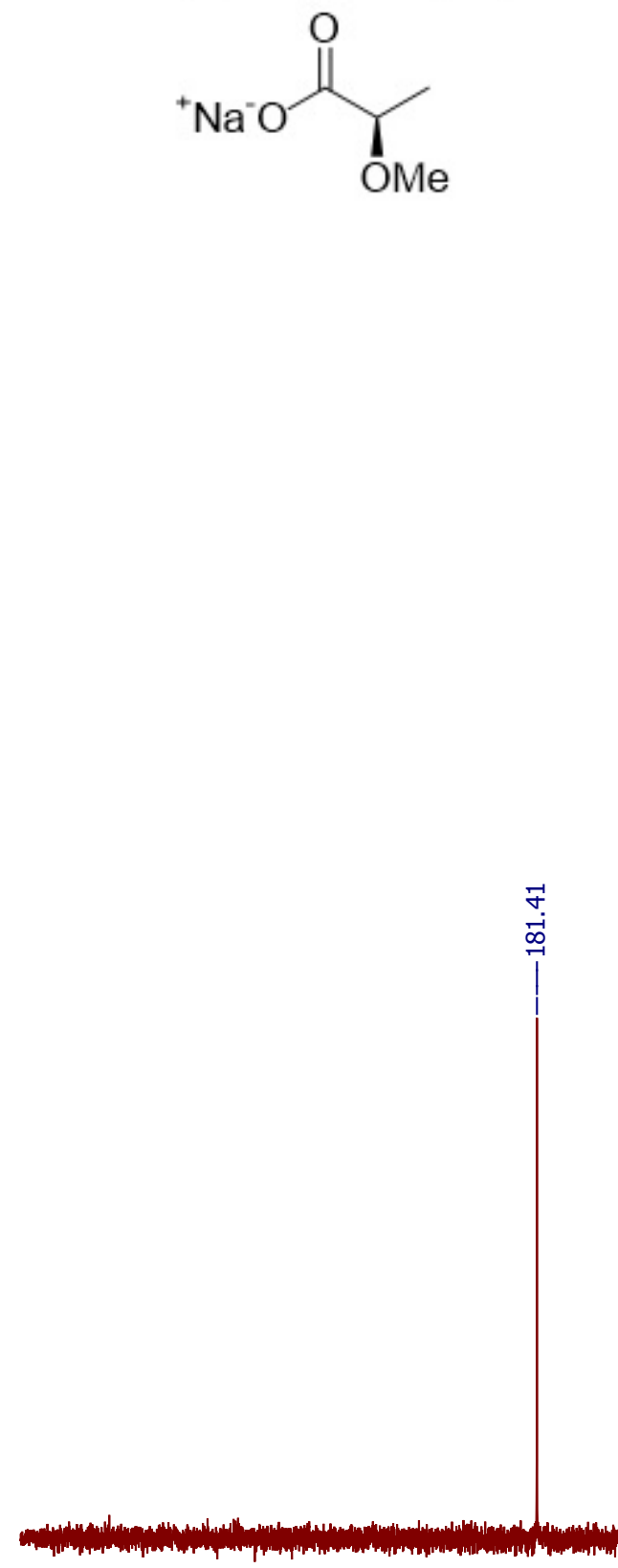
Sample Assayed for Purity by

Quantitative NMR vs $\mathrm{MeCN}$ : $91 \%$

Yield adjusted for Purity: $95 \%(40 \mathrm{~g})$
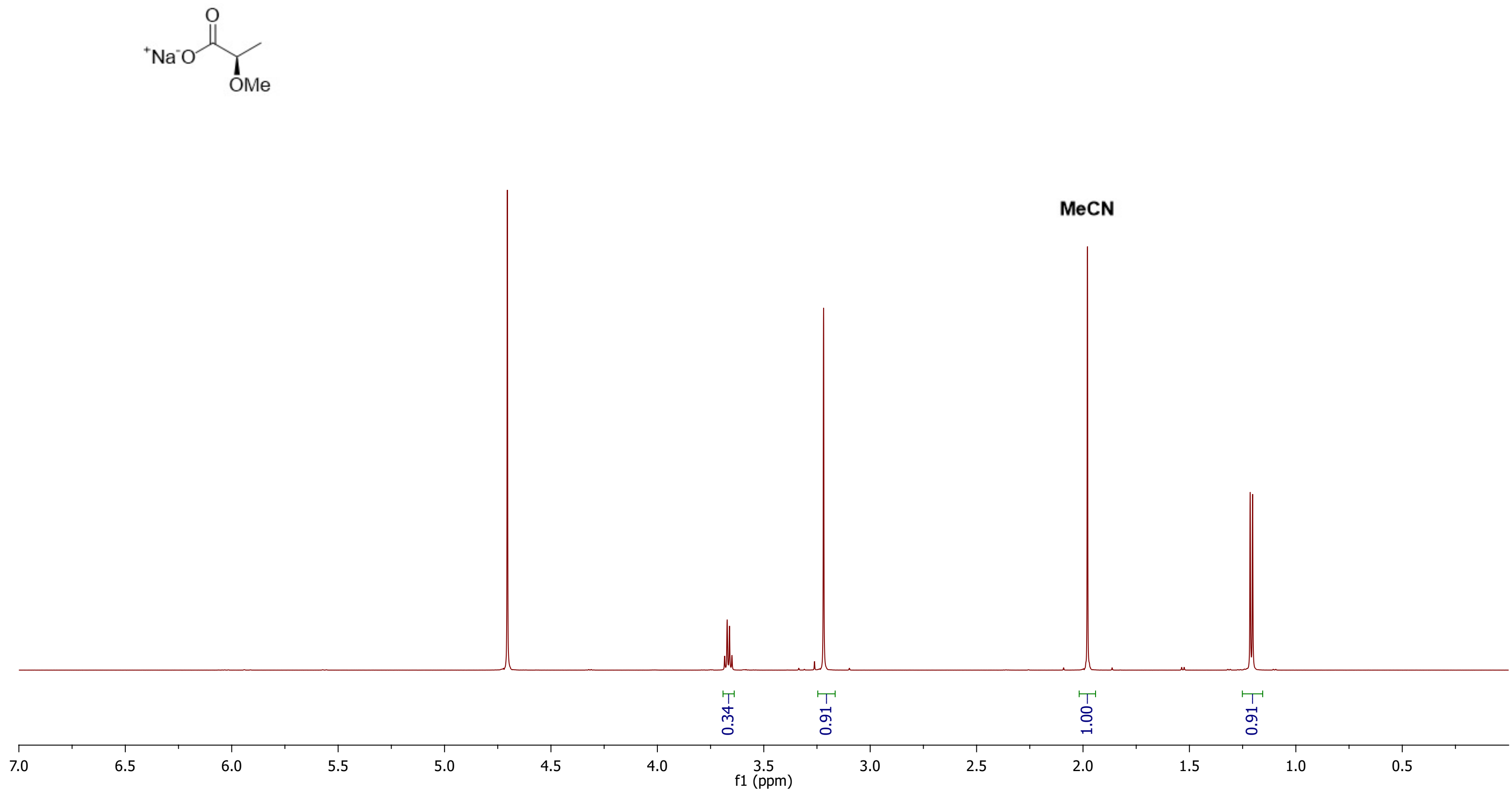


\section{DRS_508}

Run in negative mode, loss of $\mathrm{Na}$ counterion TH M-H Mass: 103.0395

Experimental M-H Mass: 103.0404

Error: 8.7 ppm

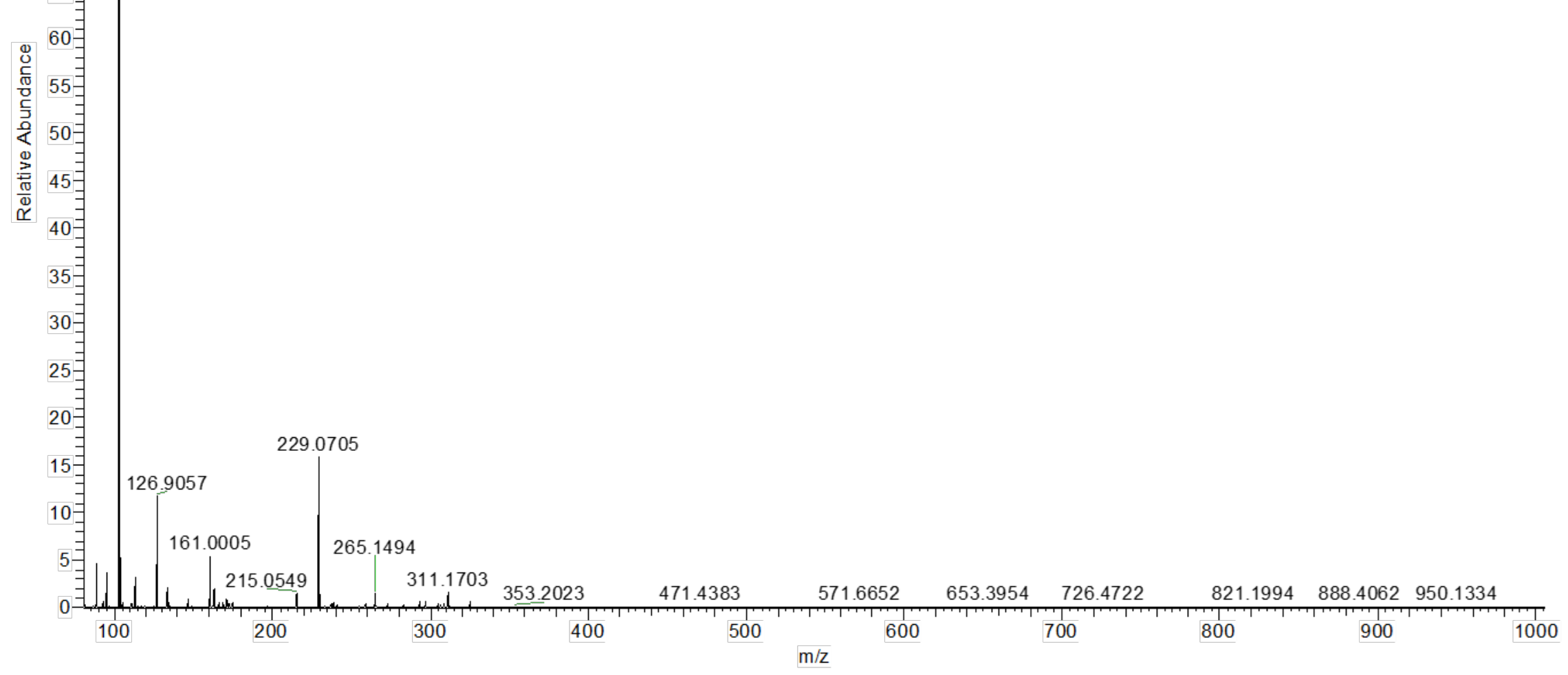




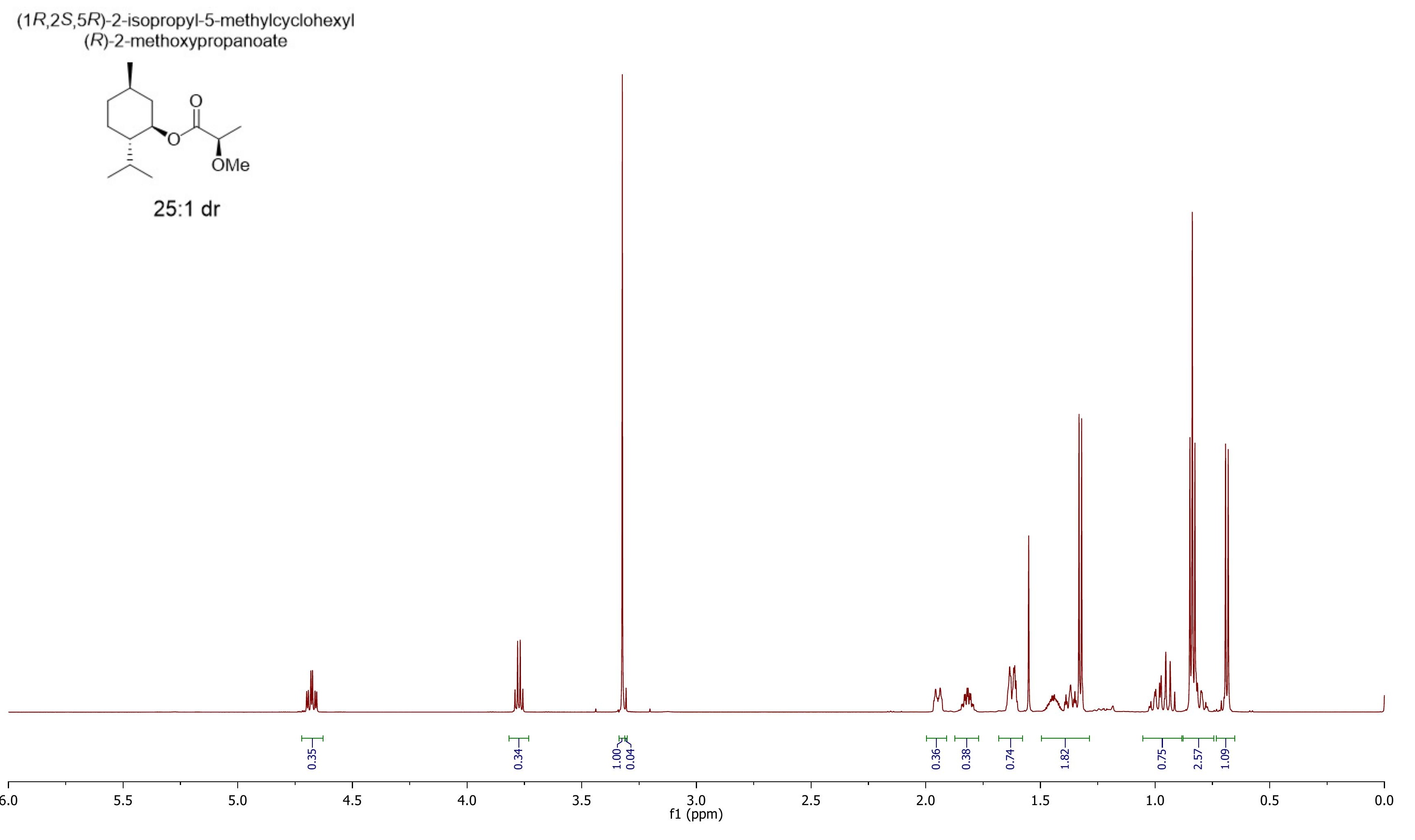




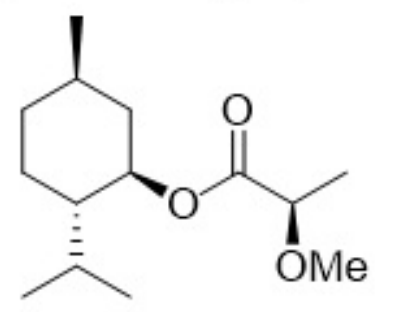

\section{$25: 1 \mathrm{dr}$}



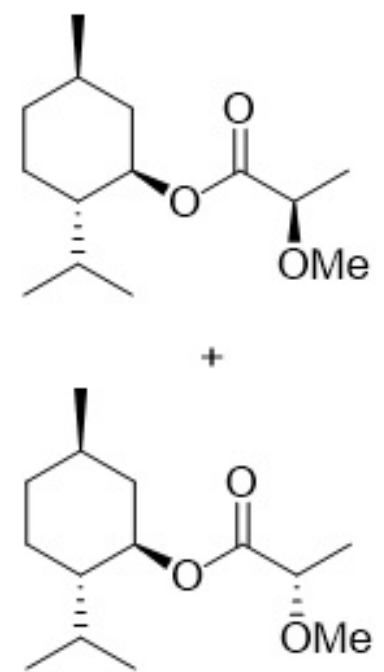

${ }^{1} \mathrm{H} \mathrm{NMR}\left(600 \mathrm{MHz}, \mathrm{CDCl}_{3}\right) \delta 4.70(\mathrm{ddd}, J=21.8,10.9,4.4 \mathrm{~Hz}, 1 \mathrm{H}), 3.77(\mathrm{qd}$ $J=6.8,4.1 \mathrm{~Hz}, 1 \mathrm{H}), 3.31(\mathrm{~d}, J=9.9 \mathrm{~Hz}, 3 \mathrm{H}), 2.00-1.87(\mathrm{~m}, 1 \mathrm{H}), 1.87-1.75$

$(\mathrm{m}, 1 \mathrm{H}), 1.62(\mathrm{dd}, J=9.5,7.1 \mathrm{~Hz}, 2 \mathrm{H}), 1.50-1.40(\mathrm{~m}, 1 \mathrm{H}), 1.40-1.29(\mathrm{~m}$,

$4 \mathrm{H}), 1.05-0.89(\mathrm{~m}, 2 \mathrm{H}), 0.86-0.75(\mathrm{~m}, 7 \mathrm{H}), 0.70(\mathrm{dd}, J=11.3,7.0 \mathrm{~Hz}, 3 \mathrm{H})$

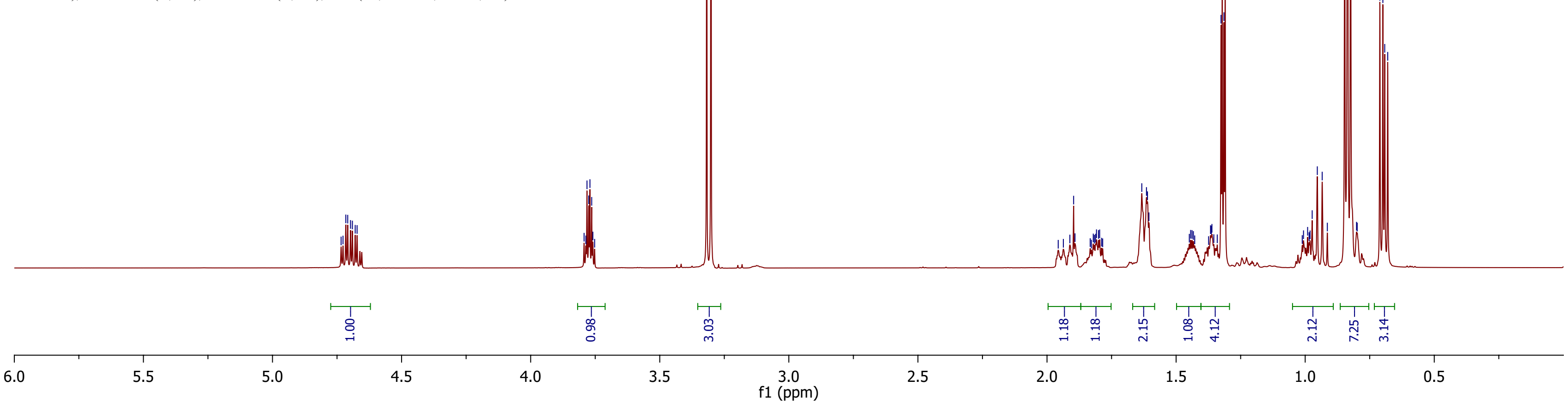



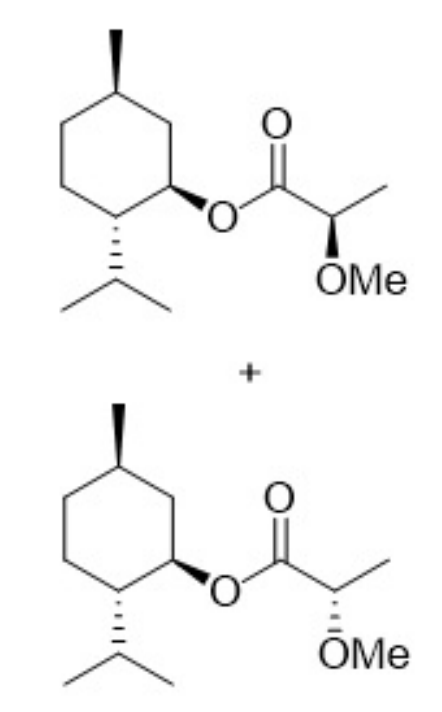

$$
\begin{array}{r}
\Gamma \\
3.7
\end{array}
$$

$\begin{array}{llll}3.65 & 3.60 & 3.55 & 3.50\end{array}$




\section{(1R,2S,5R)-2-isopropyl-5-methylcyclohexyl}

$(R)$-2-methoxypropanoate

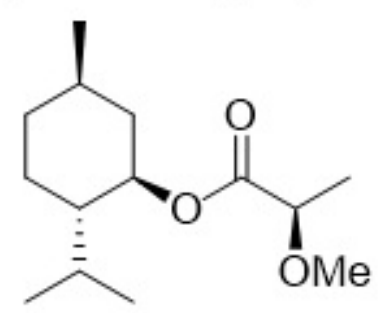

$25: 1 d r$

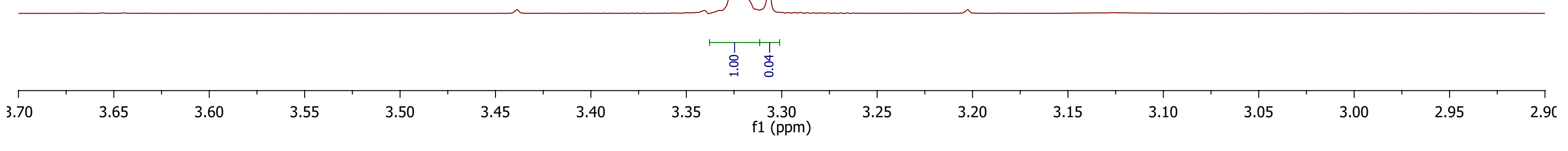




\section{Acylation to form 4a:}
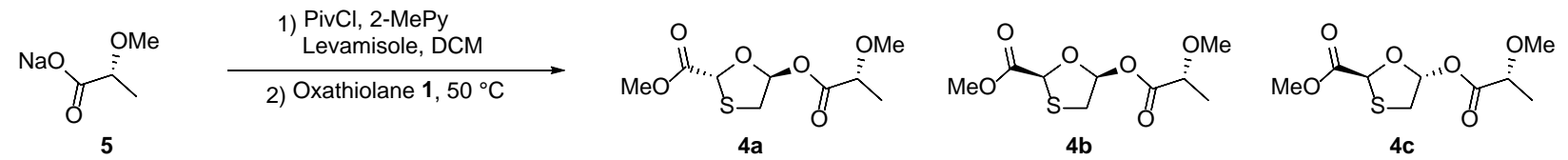

Figure S3: Acylation of oxathiolane 1 with carboxylate 5.

\section{Typical Procedure for Synthesis of Acylated Oxathiolane 4a:}

Sodium lactate salt 5 ( $6.18 \mathrm{~g}, 97.5 \mathrm{wt} \%, 47.8 \mathrm{mmol}, 1.8$ equiv.) was added to a dried round-bottom flask under nitrogen. Dichloromethane ( $25 \mathrm{~mL}, 99.8 \%$, MilliporeSigma) was charged, followed by pivaloyl chloride $(5.89 \mathrm{~mL}, 47.8 \mathrm{mmol}, 1.8$ equiv., $>98 \%$ Alfa-Aesar). A slight exotherm was observed in the reaction of pivaloyl chloride and the carboxylate. The suspension stirred at room temperature for 45 min. 2-Picoline ( $0.535 \mathrm{~mL}, 5.31 \mathrm{mmol}, 0.2$ equiv., $98 \%$ Sigma-Aldrich) was then added. After mixing, the anhydride solution was transferred via wide-bore cannula to a separate vessel containing hydroxyoxathiolane 1 (5.316 g, 82 wt\%, 26.6 mmol, 1 equiv.), levamisole hydrochloride $(95.9 \mathrm{mg}, 0.398$ $\mathrm{mmol}, 1.5 \mathrm{~mol} \%$, >99\% TCl Chemicals), and dichloromethane $(25 \mathrm{~mL})$ heated to reflux. The addition occurred over a period of 120 minutes. The reaction was kept at reflux for 3 hours after addition until reaction monitoring showed complete consumption of the hydroxyoxathiolane. The oxathiolane suspension was transferred to a separatory funnel containing $1 \mathrm{~mL}$ of conc. $\mathrm{HCl}$ diluted in $50 \mathrm{~mL}$ of $\mathrm{H}_{2} \mathrm{O}$. After vigorous mixing, the organic layer was removed, and the aqueous layer was back extracted twice with DCM ( $2 \mathrm{~mL} \times 2)$. The combined organic fractions were then washed with a combined mixture of saturated potassium carbonate solution $(50 \mathrm{~mL})$ and water $(50 \mathrm{~mL})$. The aqueous fractions were back extracted twice with DCM $(2 \mathrm{~mL} \times 2)$. The combined organic fractions were concentrated under vacuum. $9.839 \mathrm{~g}$ of product was collected and assayed ( $53 \mathrm{wt} \%, 78 \%$ yield). Toluene $(9 \mathrm{~mL})$ and hexanes $(12 \mathrm{~mL})$ were added to the crude product which was then seeded with a crystal of $4 \mathrm{a}$. The solution stood at room temperature for $2 \mathrm{hr}$ before being placed in a $-15^{\circ} \mathrm{C}$ freezer for $16 \mathrm{hr}$. The resultant crystals were then collected by vacuum filtration and washed with a solution of toluene and hexanes $(4 \mathrm{~mL}, 1: 1.33)$ cooled to $-15^{\circ} \mathrm{C}$. The crystals were dried and analyzed ( $4.311 \mathrm{~g}, 89.6 \mathrm{wt} \% \mathbf{5}, 83.7 \mathrm{wt} \% \mathbf{4 a}, 54 \%$ yield $\left.4 \mathrm{a}\right)$. When $\mathbf{4 b}$ content above $5 \%$, a second crystallization was conducted with a 1.1 .3 mixture of toluene/hexanes. ${ }^{1} \mathrm{H} \mathrm{NMR}\left(600 \mathrm{MHz}, \mathrm{CDCl}_{3}\right) \delta 6.83(\mathrm{~d}, J=4.0 \mathrm{~Hz}, 1 \mathrm{H}), 5.65(\mathrm{~s}, 1 \mathrm{H}), 3.88(\mathrm{q}, J=6.9 \mathrm{~Hz}, 1 \mathrm{H}), 3.78(\mathrm{~s}$, $3 \mathrm{H}), 3.45(\mathrm{~m}, 1 \mathrm{H}), 3.39(\mathrm{~s}, 3 \mathrm{H}), 3.19(\mathrm{~m}, 1 \mathrm{H}), 1.39(\mathrm{~d}, J=6.9 \mathrm{~Hz}, 3 \mathrm{H}) .{ }^{13} \mathrm{C} \mathrm{NMR}\left(151 \mathrm{MHz}, \mathrm{CDCl}_{3}\right) \delta 171.81(\mathrm{~s}), 169.34$ (s), 100.12 (s), $79.78(\mathrm{~s}), 75.99$ (s), 57.82 (s), 52.83 (s), 37.31 (s), 18.24 (s). HRMS: Expected M+Na m/z 273.0409, Found: 273.0398.

\section{Reaction Sensitivities-Important Notes for Reproduction:}

- Reagents must be anhydrous. Water present in reagents (sodium lactate salt, oxathiolane) will react with the anhydride, generating two equivalents of carboxylic acid. The acid will bind with catalyst (Levamisole or DMAP) and shutdown the reaction.

- The amount of pivaloyl chloride should be equal to or less than the amount of sodium lactate salt. Excess pivaloyl chloride binds to the catalyst (Levamisole or DMAP) and inhibits the reaction.

- Base: 
o Should be added to anhydride solution and not to the oxathiolane directly. Adding to the oxathiolane directly leads to decomposition of the oxathiolane presumably via ring opening.

0 Quantity can be varied depending on dryness of reagent. If reagents are very dry, only small amounts of picoline are needed to neutralize acid ( 0.1 equivalents or less). Use of less base leads to final product of lower color.

o Stronger bases such as triethylamine should not be used. Selection of TEA leads to very high levels of oxathiolane decomposition. Pyridine, 2-picoline, and 2,6-lutidine all work equally well as do certain weak inorganic bases (sodium carbonate, basic alumina, etc.).

- Order of addition: Anhydride should be added to oxathiolane. Heated oxathiolane is rapidly epimerizing at the thioacetal and anomeric positions; however, the anomeric hydroxyl group is very reactive with acylating reagents. A deficiency of anhydride relative to the oxathiolane is created by adding anhydride to the oxathiolane. Under this regime the most reactive diastereomer is acylated and the population of this diastereomer is replenished by promoting epimerization with heat.

- Rate of addition: Slow addition ( $>1 \mathrm{hr}$ ) gives the most selective acylation due to time allowed for the DKR; however, overall mass balance ( $80 \%$ acylation products) is lower than when addition is performed quickly (<30 min, $>90 \%$ acylation products). Overall yields for the desired diastereomer tend to be similar; however, crystallization of the slow addition product tends to be simpler.

- Extraction: Basic aqueous layer should be double the volume of the organic layer to dilute the carboxylate salts which are being removed. Some product will be partition into the aqueous layer when more concentrated solutions are used due to the high organic content from the carboxylates.

- Crystallization:

0 Needs to be held at room temperature for $1-2 \mathrm{hr}$ before cooling to $-15^{\circ} \mathrm{C}$. Cooling too quickly leads to lower optical purity.

o Should not be kept at $-15^{\circ} \mathrm{C}$ more than 1 day. The other trans diastereomer (major byproduct-Levamisole) is also crystalline and will eventually come out of solution, although the predominant cis diastereomer is oily (major byproduct-DMAP). 


\section{Results for Multigram Runs and Isolations:}

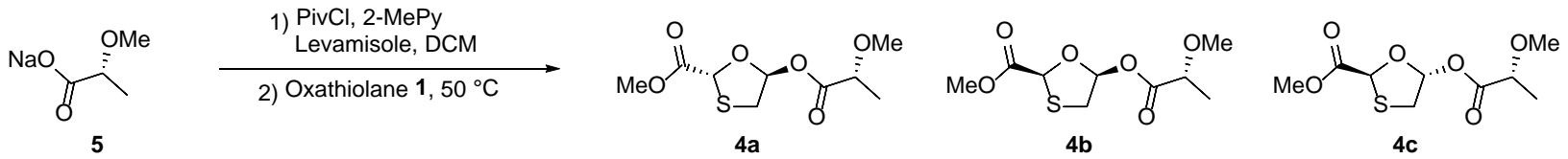

Table S9: Acylation of hydroxyoxathiolane 1 at $>1 \mathrm{~g}$ scale.

\begin{tabular}{|c|c|c|c|c|c|c|c|c|c|c|c|}
\hline Entry & Scale & Addition (Time) & $\begin{array}{c}\text { Oxathiolane } \\
\text { Purified? }\end{array}$ & Purification Stage & $\begin{array}{c}\text { Amount } \\
\text { Recovered }\end{array}$ & $\begin{array}{c}\text { Purity } \\
\text { (\%) }\end{array}$ & $4 a(\%)$ & $4 b(\%)$ & $4 c(\%)$ & Yield 4 & Yield 4a \\
\hline \multirow[t]{3}{*}{1} & $8 \mathrm{~g}$ & $120 \mathrm{~min}$ & No & Extraction & 11.934 & 55.2 & 57 & 15 & 6 & $79 \%$ & $57 \%$ \\
\hline & & & & $1^{\text {st }}$ Cryst. & 6.854 & 71.8 & 50 & 6 & 3 & $59 \%$ & $50 \%$ \\
\hline & & & & $2^{\text {nd }}$ Cryst. & 4.904 & 94.0 & 52 & 2 & 1 & $55 \%$ & $52 \%$ \\
\hline \multirow[t]{3}{*}{2} & $5.3 \mathrm{~g}$ & $120 \mathrm{~min}$ & Yes & EOR & - & - & 58 & 15 & 6 & $79 \%$ & $58 \%$ \\
\hline & & & & Extraction & 9.839 & 52.7 & 57 & 16 & 5 & $78 \%$ & $57 \%$ \\
\hline & & & & $1^{\text {st }}$ Cryst. & 4.241 & 89.6 & 54 & 2 & 2 & $57 \%$ & $54 \%$ \\
\hline \multirow[t]{3}{*}{3} & $5 \mathrm{~g}$ & $240 \mathrm{~min}$ & Yes & Extraction & 6.49 & 60.0 & 58 & 14 & 5.2 & $78 \%$ & $58 \%$ \\
\hline & & & & $1^{\text {st }}$ Cryst. & 4.101 & 74.4 & 53 & 5.3 & 2.4 & $61 \%$ & $53 \%$ \\
\hline & & & & $2^{\text {nd }}$ Cryst. & 3.194 & 92.0 & 54 & 3.1 & 1.8 & $59 \%$ & $54 \%$ \\
\hline \multirow[t]{4}{*}{4} & $1 \mathrm{~g}$ & $240 \mathrm{~min}$ & Yes & EOR & - & - & 60 & 16 & 6 & $82 \%$ & $60 \%$ \\
\hline & & & & Extraction & 1.24 & 62 & 56 & 15 & 6 & $77 \%$ & $56 \%$ \\
\hline & & & & $1^{\text {st }}$ Cryst. & 0.792 & 79 & 54 & 6 & 3 & $63 \%$ & $54 \%$ \\
\hline & & & & $2^{\text {nd }}$ Cryst. & 0.574 & 95 & 52 & 2 & 1 & $55 \%$ & $52 \%$ \\
\hline 5 & $1 \mathrm{~g}$ & $170 \mathrm{~min}$ & Yes & EOR & - & - & 58 & 16 & 6 & $82 \%$ & $58 \%$ \\
\hline 6 & $1 \mathrm{~g}$ & $120 \mathrm{~min}$ & Yes & EOR & - & - & 57 & 16 & 5 & $78 \%$ & $57 \%$ \\
\hline 7 & $1 \mathrm{~g}$ & $120 \mathrm{~min}$ & Yes & EOR & - & - & 58 & 16 & 5 & $79 \%$ & $58 \%$ \\
\hline 8 & $10 \mathrm{~g}$ & $20 \mathrm{~min}$ & Yes & EOR & - & - & 59 & 24 & 8 & $92 \%$ & $59 \%$ \\
\hline 9 & $10 \mathrm{~g}$ & $15 \mathrm{~min}$ & Yes & EOR & - & - & 58 & 25 & 8 & $92 \%$ & $58 \%$ \\
\hline
\end{tabular}




\section{Results from Initial Optimization with DMAP:}

Typical procedure for screening with DMAP as catalyst: Sodium lactate salt 5 (30.0 mg, 98 wt\%, 0.233 mmol, 1.2 equiv.), $\mathrm{CDCl}_{3}(0.150 \mathrm{~mL})$, and pivaloyl chloride ( $28.7 \mu \mathrm{L}, 0.233 \mathrm{mmol}, 1.2$ equiv.) were loaded into a $1 \mathrm{~mL}$ vial under an atmosphere of dried air. The mixture was stirred for 5 minutes, and then DMAP (1.2 mg, $9.7 \mu \mathrm{mol}, 5 \mathrm{~mol} \%)$, 2,6-lutidine ( $27.0 \mu \mathrm{L}, 0.233 \mathrm{mmol}, 1.2$ equiv.), and oxathiolane 1 (35.8 $\mathrm{mg}, 89 \mathrm{wt} \%, 0.194 \mathrm{mmol}, 1$ equiv.) were added sequentially. Mesitylene was added as internal standard and the reaction was assayed by quantitative ${ }^{1} \mathrm{H}$ NMR after stirring for $16 \mathrm{hr}$.

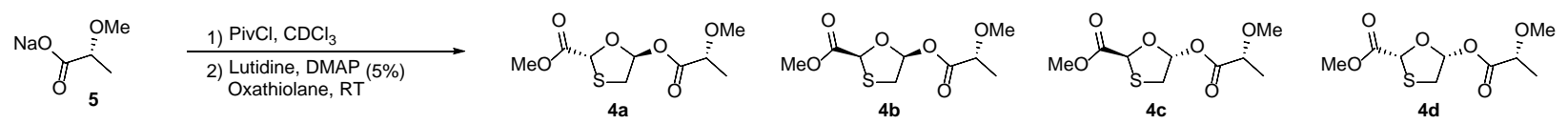

Table S10: Optimization of acylation with DMAP as a catalyst.

\begin{tabular}{|c|c|c|c|c|c|c|}
\hline Entry & Experiment & $\begin{array}{l}\text { Yield } \\
4(\%)\end{array}$ & $\begin{array}{c}\text { Yield 4a } \\
(\%)\end{array}$ & $\begin{array}{c}\text { Mass Balance } \\
(\mathrm{SM}+\mathrm{P}, \%)\end{array}$ & $\begin{array}{c}\text { Trans:Cis } \\
(4 a+4 c / 4 b+4 d)\end{array}$ & $\begin{array}{c}2 R: 2 S \\
(4 a+4 b / 4 c+4 d)\end{array}$ \\
\hline 1 & Lutidine as base & & & & 4.71 & 2.00 \\
\hline 2 & Pivalic Acid \& TEA as base & & & & 2.27 & 1.33 \\
\hline 3 & Pyridine as base & & & & 4.17 & 1.94 \\
\hline 4 & TEA as base & & & & - & - \\
\hline 5 & $-15^{\circ} \mathrm{C}$ & 58 & 30 & 69 & 5.44 & 1.58 \\
\hline 6 & $20^{\circ} \mathrm{C}$ & 61 & 34 & 61 & 5.10 & 2.00 \\
\hline 7 & $50{ }^{\circ} \mathrm{C}$ & 56 & 32 & 56 & 5.22 & 2.13 \\
\hline 8 & RT, PivCl added portionwise last & 56 & 33 & 67 & 5.22 & 2.36 \\
\hline 9 & 1/4 Charge Lutidine & 68 & 37 & 68 & 5.18 & 1.85 \\
\hline 10 & Pyridine as base & 68 & 38 & 68 & 5.80 & 1.90 \\
\hline 11 & 2-Picoline as base & 69 & 37 & 74 & 5.90 & 1.68 \\
\hline 12 & No Lutidine, 2 Equiv. LA & 86 & 43 & 86 & 4.38 & 1.59 \\
\hline 13 & No DMAP & 29 & 12 & 92 & 2.22 & 1.50 \\
\hline \multirow[t]{2}{*}{14} & $70{ }^{\circ} \mathrm{C}$, No Catalyst or Base & 83 & 18 & 90 & 1.31 & 0.62 \\
\hline & Order of Addition: & & & & & \\
\hline $15 a$ & Salt $\rightarrow$ Piv $\rightarrow$ Lut $\rightarrow$ Ox. $\rightarrow$ DMAP & 83 & 46 & 83 & 6.55 & 1.77 \\
\hline $15 b$ & Salt $\rightarrow$ Piv $\rightarrow$ Ox. $\rightarrow$ Lut $\rightarrow$ DMAP & 80 & 44 & 80 & 7.00 & 1.69 \\
\hline $15 c$ & Salt $\rightarrow$ Ox. $\rightarrow$ Piv $\rightarrow$ Lut $\rightarrow$ DMAP & 76 & 35 & 76 & 3.22 & 1.52 \\
\hline $15 d$ & Portionwise Addition of PivCl/Lutidine Last & 57 & 33 & 57 & 4.18 & 2.54 \\
\hline 16 & 30 min (Time for Anhydride Generation) & 84 & 45 & 84 & 5.46 & 1.73 \\
\hline 17 & 70 min (Time for Anhydride Generation) & 83 & 44 & 83 & 5.38 & 1.69 \\
\hline 18 & 95 min (Time for Anhydride Generation) & 87 & 45 & 87 & 5.21 & 1.61 \\
\hline 19 & 125 min (Time for Anhydride Generation) & 87 & 46 & 87 & 5.69 & 1.64 \\
\hline 20 & 240 min (Time for Anhydride Generation) & 79 & 41 & 79 & 5.08 & 1.64 \\
\hline 21 & Anhydride Slowly Added to Oxathiolane & 74 & 48 & 88 & 7.22 & 2.82 \\
\hline 22 & 1.4 Equiv. Lactate/PivCl & 89 & 47 & 89 & 5.36 & 1.68 \\
\hline 23 & 1.6 Equiv. Lactate/PivCl & 91 & 45 & 91 & 4.69 & 1.50 \\
\hline 24 & 1.8 Equiv. Lactate/PivCl & 89 & 44 & 89 & 4.93 & 1.47 \\
\hline 25 & 1.8 Equiv. Lactate/PivCl, 15 Vol. Solvent & 93 & 51 & 93 & 6.15 & 1.76 \\
\hline 26 & 1.8 Equiv. Lactate/PivCl, Reverse Addition & 90 & 57 & 90 & 7.18 & 2.59 \\
\hline 27 & $\mathrm{BzCl}$ instead of $\mathrm{PivCl}, 1.4$ Equiv. & 87 & 41 & 87 & 4.12 & 1.41 \\
\hline
\end{tabular}




\begin{tabular}{|c|c|c|c|c|c|c|}
\hline 28 & $\mathrm{BzCl}$ instead of PivCl, 1.6 Equiv. & 93 & 38 & 93 & 2.88 & 1.23 \\
\hline 29 & $\mathrm{BzCl}$ instead of PivCl, 1.8 Equiv. & 91 & 39 & 91 & 3.55 & 1.22 \\
\hline 30 & DCM (Solvent) & 85 & 44 & 85 & 4.31 & 1.76 \\
\hline 31 & Dioxane (Solvent) & 83 & 46 & 83 & 4.53 & 2.09 \\
\hline 32 & MeCN (Solvent) & 70 & 36 & 70 & 4.00 & 1.80 \\
\hline 33 & THF (Solvent) & 85 & 48 & 85 & 6.73 & 1.85 \\
\hline 34 & CDCl3 (Solvent) & 88 & 46 & 88 & 4.87 & 1.70 \\
\hline 35 & DMF (Solvent) & 59 & 36 & 59 & 7.43 & 2.25 \\
\hline 36 & Toluene (Solvent) & 83 & 45 & 83 & 4.93 & 1.88 \\
\hline
\end{tabular}

\section{Results from Initial Optimization with Levamisole:}

Typical procedure for screening with Levamisole as catalyst: Sodium lactate salt 5 (30.0 mg, 87.0 wt\%, $0.207 \mathrm{mmol}, 1.4$ equiv.) $\mathrm{CDCl}_{3}(0.150 \mathrm{~mL})$, and pivaloyl chloride $(25.5 \mu \mathrm{L}, 0.207 \mathrm{mmol}, 1.4$ equiv.) were loaded into a $1 \mathrm{~mL}$ vial in sequence under an atmosphere of dried air. The mixture was stirred for 5 minutes, and then levamisole hydrochloride $(1.78 \mathrm{mg}, 0.00739 \mathrm{mmol}, 5 \mathrm{~mol} \%), 2,6$-lutidine $(6.42 \mu \mathrm{L}$, $0.0554 \mathrm{mmol}, 0.375$ equiv.), and oxathiolane 1 ( $27.3 \mathrm{mg}, 89.0 \mathrm{wt} \%, 0.148 \mathrm{mmol}, 1$ equiv.) were added sequentially. Mesitylene was added as internal standard and the reaction was assayed by quantitative ${ }^{1} \mathrm{H}$ NMR after stirring for $16 \mathrm{hr}$.

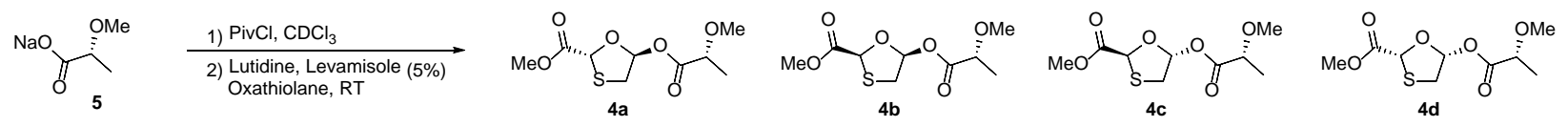

Table S11: Optimizing Acylation of 1 with levamisole as a catalyst.

\begin{tabular}{|c|c|c|c|c|c|c|}
\hline Entry & Experiment & $\begin{array}{c}\text { Yield } 4 \\
(\%)\end{array}$ & $\begin{array}{c}\text { Yield } \\
4 a(\%) \\
\end{array}$ & $\begin{array}{c}\text { Mass Balance } \\
(\mathrm{SM}+\mathrm{P}, \%)\end{array}$ & $\begin{array}{c}\text { Trans:Cis } \\
(4 a+4 c / 4 b+4 d)\end{array}$ & $\begin{array}{c}2 R: 2 S \\
(4 a+4 b / 4 c+4 d)\end{array}$ \\
\hline 1 & Pyridine as base & 81 & 46 & 81 & 2.12 & 8.00 \\
\hline 2 & Lutidine as base & 84 & 48 & 84 & 2.00 & 9.50 \\
\hline 3 & Lutidine + 5 mol\% KOtBu & 84 & 46 & 84 & 1.71 & 11.00 \\
\hline 4 & $\mathrm{~K} 2 \mathrm{CO} 3$ as base & 80 & 45 & 80 & 2.08 & 7.89 \\
\hline 5 & Slow Addition of Anhyd. to Oxathiolane & 72 & 51 & 92 & 3.80 & 11.00 \\
\hline 6 & 15 Volumes Solvent & 85 & 47 & 85 & 1.83 & 9.63 \\
\hline 7 & Low Temp $\left(0^{\circ} \mathrm{C}\right)$ & 78 & 43 & 78 & 2.00 & 7.67 \\
\hline 8 & $20 \%$ Conversion, $-40^{\circ} \mathrm{C}$ & & & & 3.80 & 23.00 \\
\hline 9 & $40 \%$ Conversion, $-40^{\circ} \mathrm{C}$ & & & & 3.94 & 12.58 \\
\hline 10 & 1.6 Equiv. NaLactate, PivCl & 91 & 57 & 91 & 2.22 & 9.00 \\
\hline 11 & 1.8 Equiv. NaLactate, PivCl & 87 & 55 & 87 & 2.46 & 8.83 \\
\hline 12 & 5 mol\% Levamisole & 79 & 54 & 79 & 2.95 & 14.80 \\
\hline 13 & 10 mol\% Levamisole & 92 & 53 & 92 & 1.88 & 12.14 \\
\hline 14 & 20 mol\% Levamisole & 83 & 54 & 90 & 2.61 & 12.83 \\
\hline 15 & Dioxane & 25 & 16 & 95 & 2.57 & 11.50 \\
\hline 16 & Toluene & 83 & 52 & 90 & 2.46 & 10.86 \\
\hline 17 & $\mathrm{CDCl} 3$ & 86 & 57 & 95 & 2.74 & 13.33 \\
\hline 18 & NMP & 0 & 0 & 97 & - & - \\
\hline 19 & NMP/CDCl3 & 44 & 29 & 52 & 3.40 & 7.80 \\
\hline
\end{tabular}


$20 \mathrm{DMF} / \mathrm{CDCl} 3$

$2140^{\circ} \mathrm{C}$, Slow Addition, 1.6 equiv. Anhyd.

\begin{tabular}{|c|c|c|c|c|}
\hline 40 & 28 & 47 & 4.00 & 9.00 \\
\hline 92 & 66 & 92 & 4.11 & 10.5 \\
94 & 66 & 100 & 3.48 & 12.43 \\
\hline
\end{tabular}

$2240^{\circ} \mathrm{C}$, Slow Addition, 1 wt\% Levamisole 


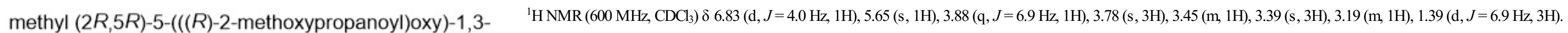
oxathiolane-2-carboxylate<smiles>COC(=O)[C@H]1O[C@@H](OC(=O)[C@H](C)OC)CS1</smiles>

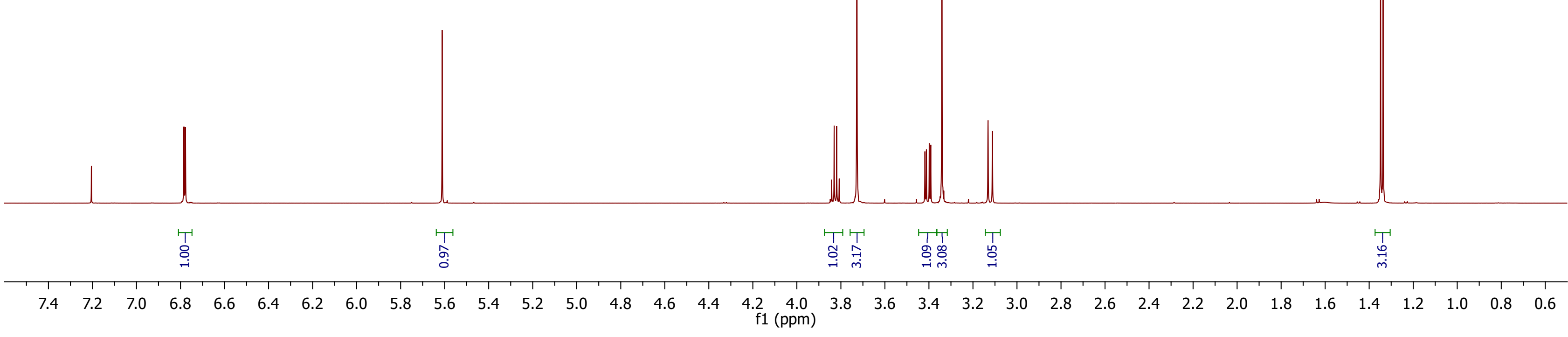


methyl (2R,5R)-5-(((R)-2-methoxypropanoyl)oxy)-1,3oxathiolane-2-carboxylate

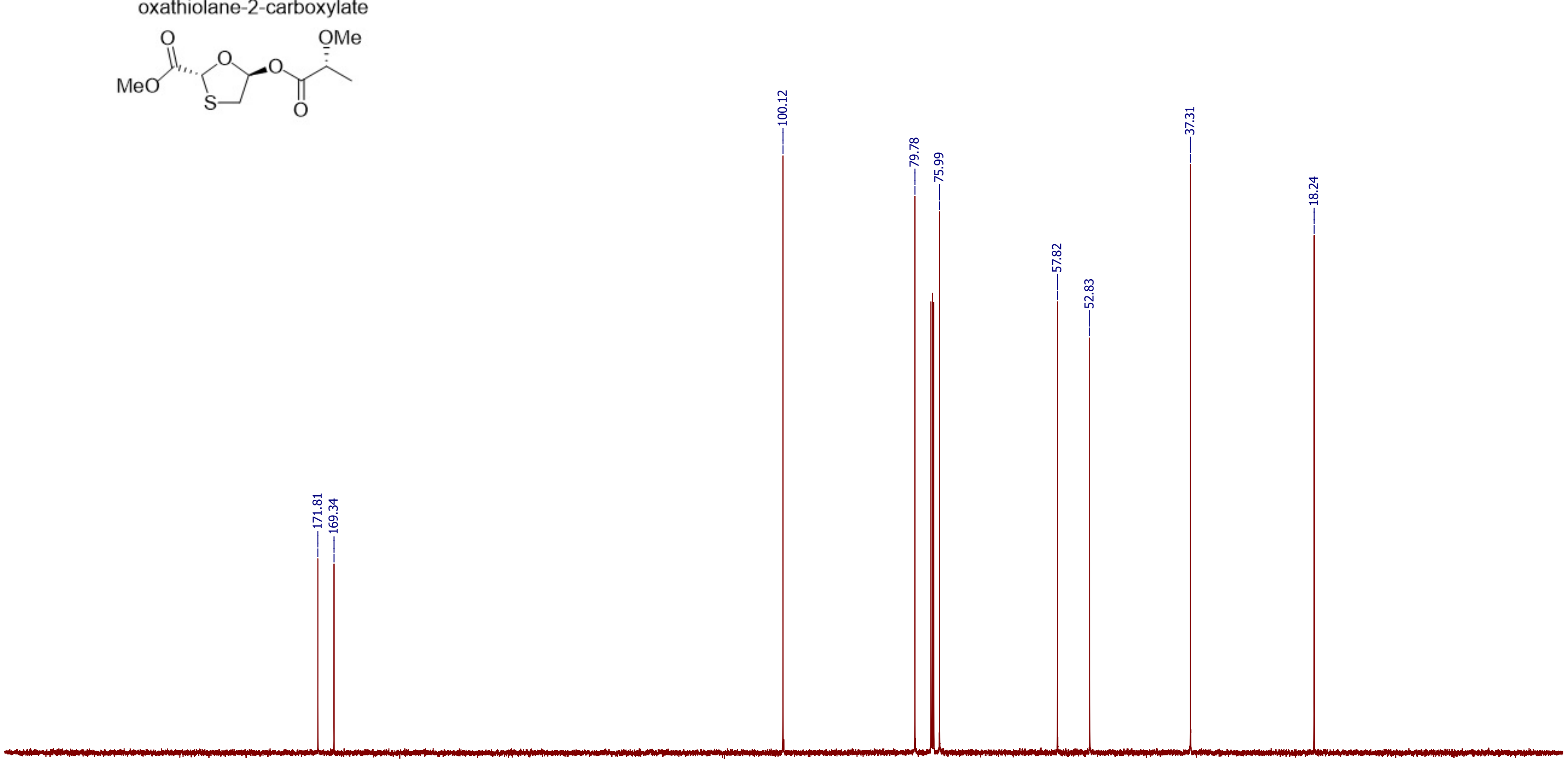




\section{Acylation Catalyzed by DMAP}

methyl $(2 R, 5 R)-5-(((R)-2-$ methoxypropanoyl)oxy)-1,3 oxathiolane-2-carboxylate

methyl (2S,5S)-5-(((R)-2-
methoxypropanoyl)oxy)-1,3 methoxypropanoyl)oxy)-1,3-
oxathiolane-2-carboxylate

methyl (2S,5R)-5-(((R)-2methoxypropanoyl)oxy)-1,3oxathiolane-2-carboxylate

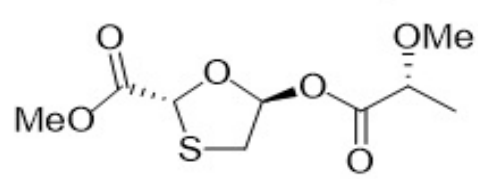

Product

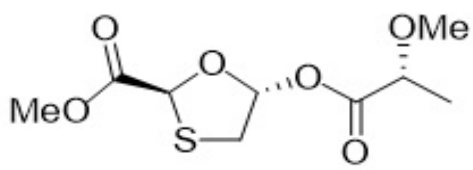

Major Byproduct

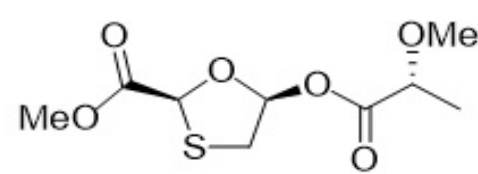

Minor Byproduct

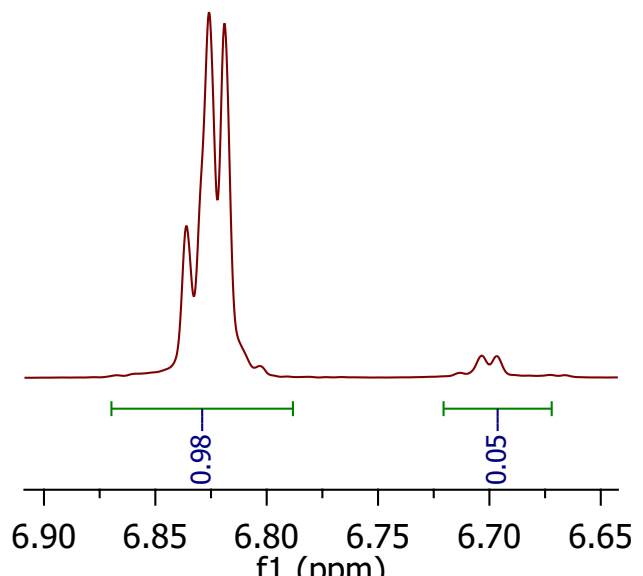

f1 (ppm)
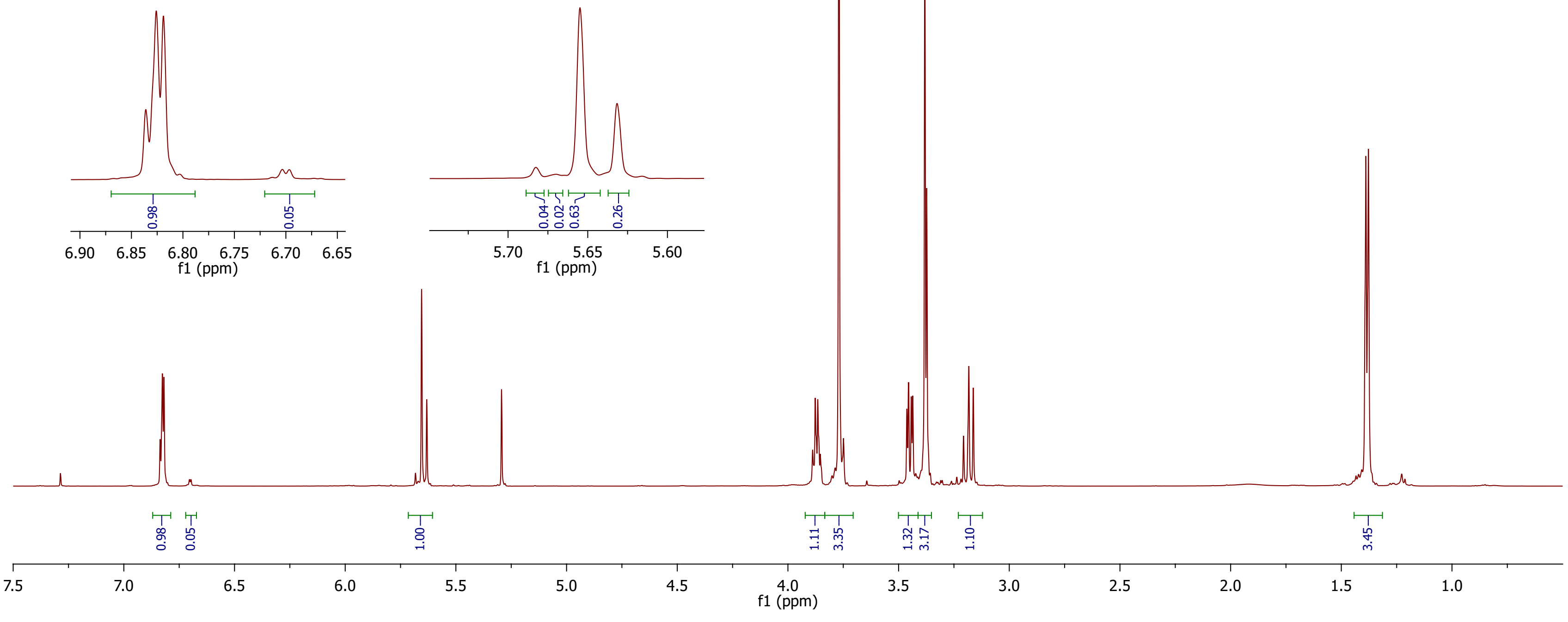

$1(\mathrm{ppm})$ 


\section{Acylation Catalyzed by Levamisole}

methyl $(2 R, 5 R)-5-(((R)-2-$ methoxypropanoyl)oxy)-1,3-

oxathiolane-2-carboxylate

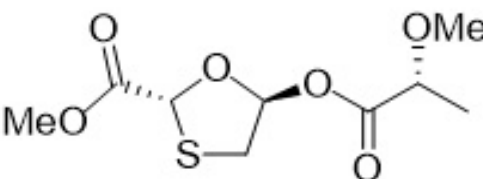

Product methyl (2S,5R)-5-(((R)-2 methoxypropanoyl)oxy)-1,3-

oxathiolane-2-carboxylate

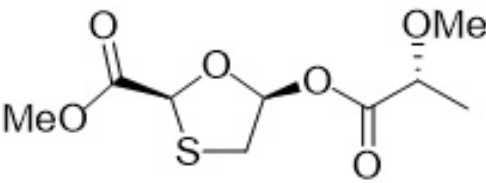

Major Byproduct methyl (2S,5S)-5-(((R)-2-

methoxypropanoyl)oxy)-1,3-

oxathiolane-2-carboxylate

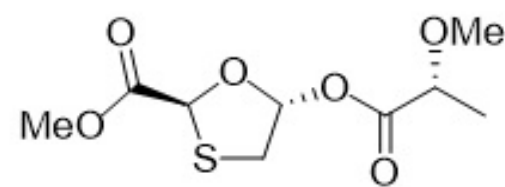

Minor Byproduct
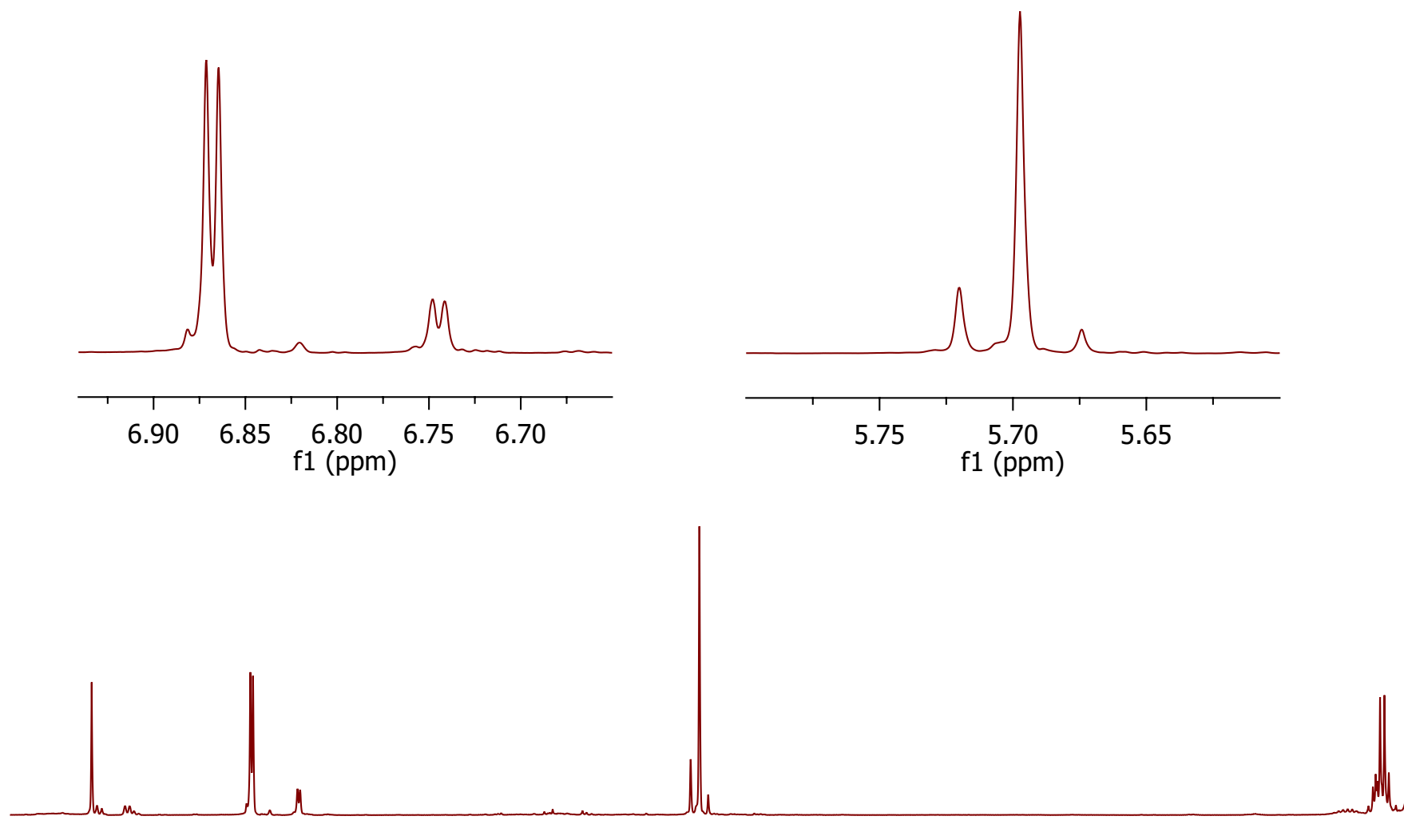

( ( 
In Process Control: Quantitative NMR Assayed Against Naphthalene

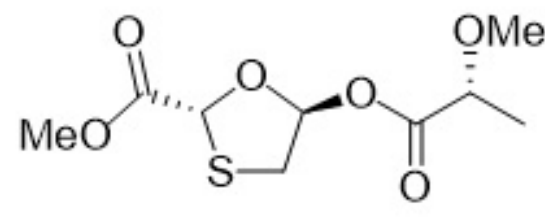

Product: $67 \%$

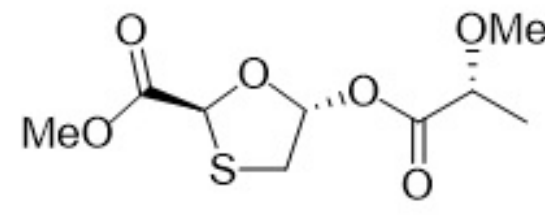

Major Byproduct: $19 \%$

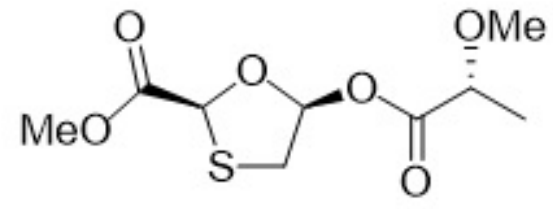

Minor Byproduct: $7 \%$

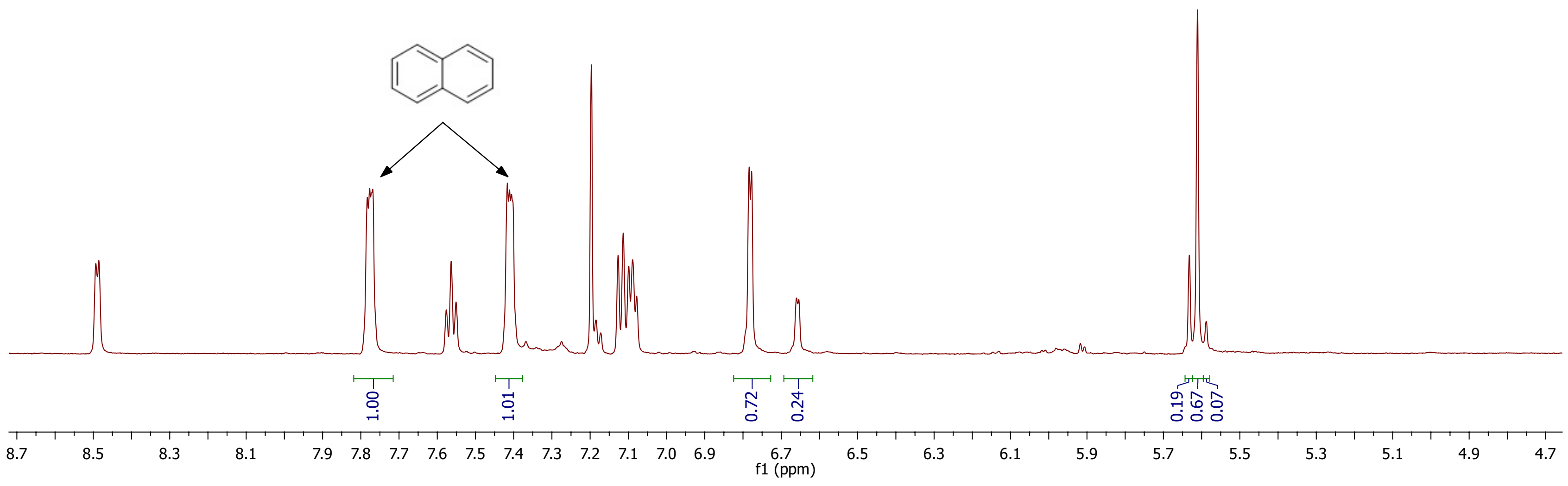




\section{After Crystallization}

methyl (2R,5R)-5-(((R)-2-methoxypropanoyl)oxy)-1,3oxathiolane-2-carboxylate
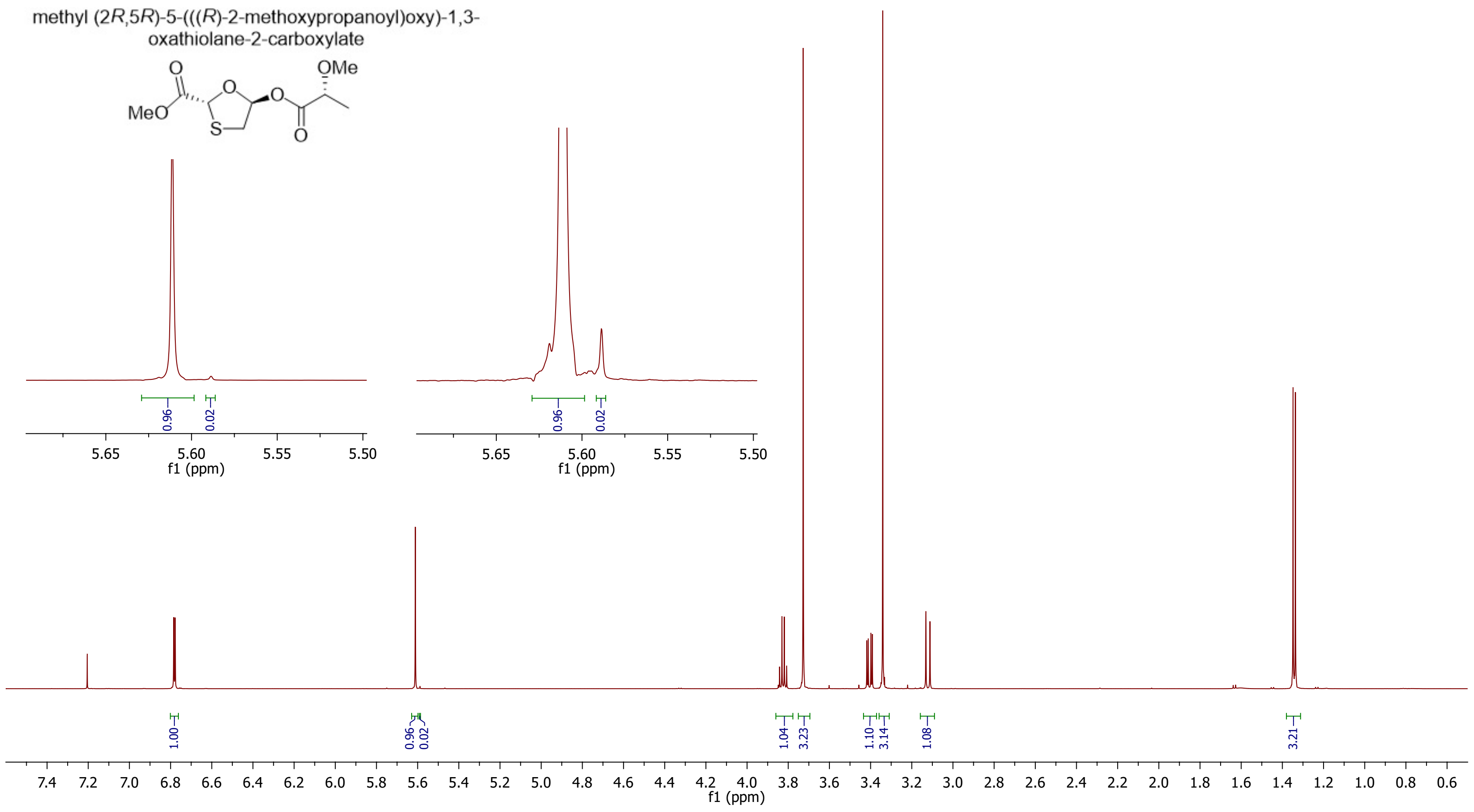


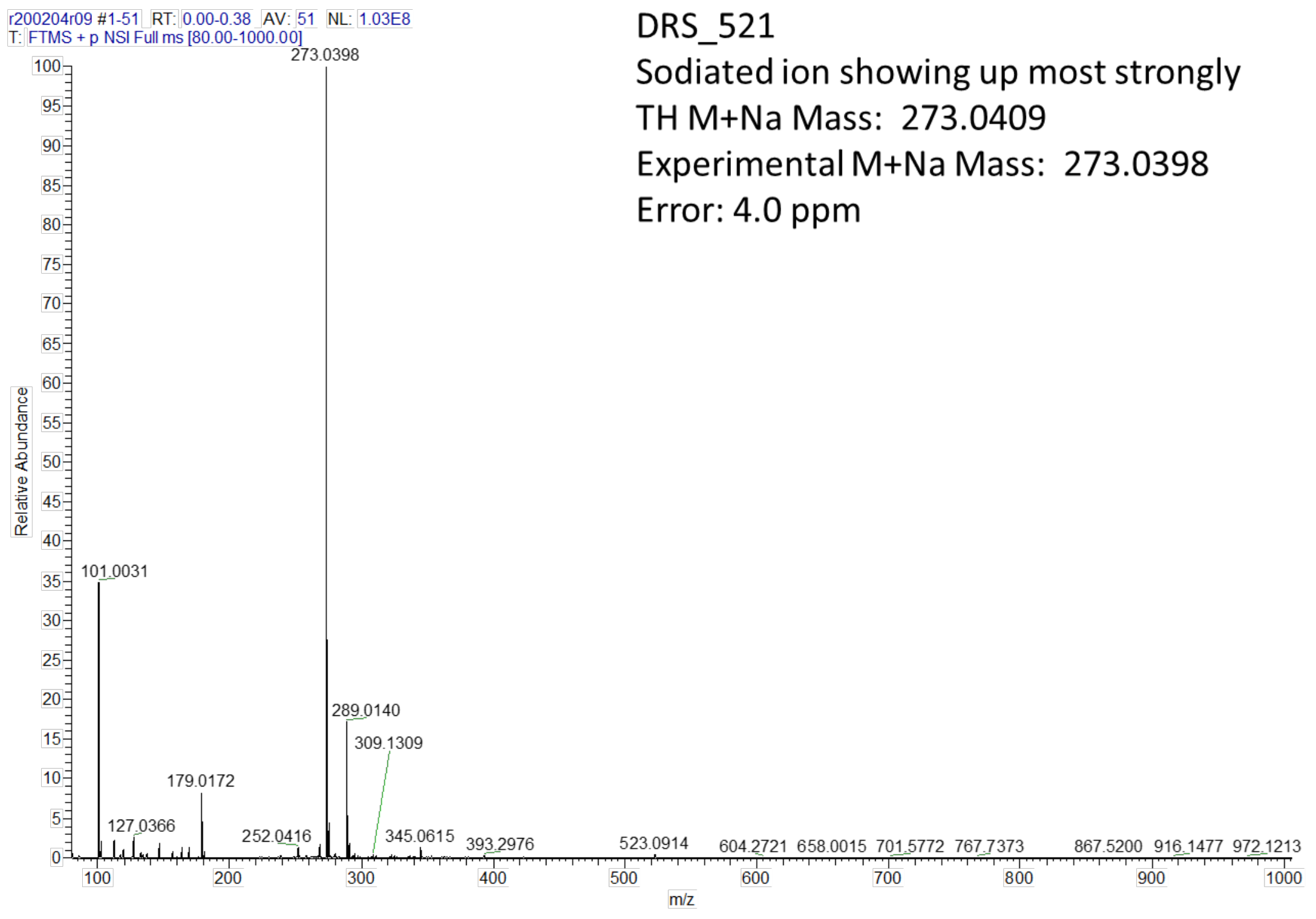




\section{Crystal Data and Experimental (4a)}
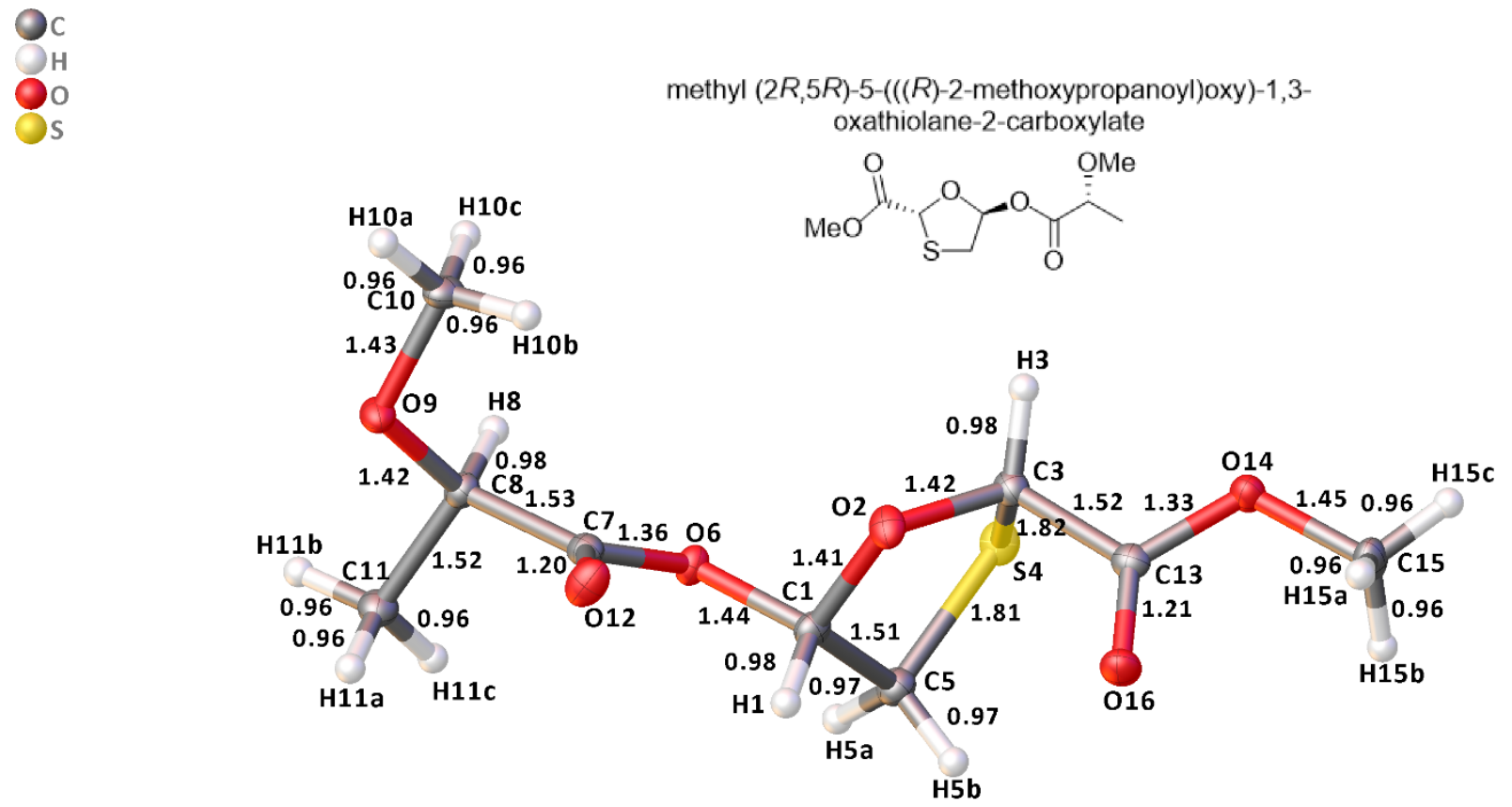

Figure S4: Depiction of 4a with data obtained from X-Ray analysis.

Sample of 4a was recrystallized from a toluene/hexanes mixture (1:1). A suitable crystal was selected and MiTeGen Microloop on a XtaLAB AFC11 (RINC): quarter-chi single diffractometer. The crystal was kept at 100.02(11) K during data collection. Using Olex2 [1], the structure was solved with the ShelXT [2] structure solution program using Intrinsic Phasing and refined with the olex2.refine [3] refinement package using Gauss-Newton minimisation. Crystallographic data and structural refinement parameters are given below. Crystallographic data has been deposited with the Cambridge Crystallographic Data Centre (CCDC) with the depository number: CCDC 1980798. This data is available from the CCDC via www.ccdc.cam.ac.uk/.

1. Dolomanov, O.V., Bourhis, L.J., Gildea, R.J, Howard, J.A.K. \& Puschmann, H. J. Appl. Cryst., 2009, 42, 339-341.

2. Sheldrick, G.M. Acta Cryst., 2015, A71, 3-8.

3. Bourhis, L.J., Dolomanov, O.V., Gildea, R.J., Howard, J.A.K., Puschmann, H. Acta Cryst., 2015, A71, 59-75.

\section{Crystal structure determination}

Crystal Data for $\mathrm{C}_{9} \mathrm{H}_{14} \mathrm{O}_{6} \mathrm{~S}(M=250.27 \mathrm{~g} / \mathrm{mol})$ : monoclinic, space group $\mathrm{P} 2_{1}$ (no. 4), $a=9.9244(2) \AA, b=$ 5.4099(1) $\AA, c=11.2454(2) \AA, b=106.975(2)^{\circ}, V=577.46(2) \AA^{3}, Z=2, T=100.02(11) K, \mu(C u K \alpha)=2.637$ $\mathrm{mm}^{-1}$, Dcalc $=1.4392 \mathrm{~g} / \mathrm{cm}^{3}, 8434$ reflections measured $\left(8.22^{\circ} \leq 2 \Theta \leq 133.16^{\circ}\right), 1809$ unique $\left(R_{\text {int }}=\right.$ $\left.0.0394, R_{\text {sigma }}=0.0173\right)$ which were used in all calculations. The final $R_{1}$ was $0.0347(\mathrm{I}>=2 \mathrm{u}(\mathrm{I}))$ and $w R_{2}$ was 0.0911 (all data). 
Table S12: Select Crystal data and structure refinement

\begin{tabular}{|c|c|}
\hline Empirical formula & $\mathrm{C}_{9} \mathrm{H}_{14} \mathrm{O}_{6} \mathrm{~S}$ \\
\hline Formula weight & 250.27 \\
\hline Temperature/K & $100.02(11)$ \\
\hline Crystal system & monoclinic \\
\hline Space group & $\mathrm{P} 2_{1}$ \\
\hline $\mathrm{a} / \AA$ & $9.9244(2)$ \\
\hline $\mathrm{b} / \AA ̊ \AA$ & $5.4099(1)$ \\
\hline$c / \AA$ & $11.2454(2)$ \\
\hline$\alpha /^{\circ}$ & 90 \\
\hline$\beta /^{\circ}$ & $106.975(2)$ \\
\hline$\gamma /{ }^{\circ}$ & 90 \\
\hline Volume/Å ${ }^{3}$ & $577.46(2)$ \\
\hline Z & 2 \\
\hline$\rho_{\text {calc }} g / \mathrm{cm}^{3}$ & 1.4392 \\
\hline$\mu / \mathrm{mm}^{-1}$ & 2.637 \\
\hline$F(000)$ & 265.6 \\
\hline Crystal size $/ \mathrm{mm}^{3}$ & $0.521 \times 0.237 \times 0.205$ \\
\hline Radiation & Cu Ka $(\lambda=1.54184)$ \\
\hline $2 \Theta$ range for data collection ${ }^{\circ}$ & 8.22 to 133.16 \\
\hline Index ranges & $-12 \leq h \leq 12,-6 \leq k \leq 5,-13 \leq \mathrm{I} \leq 14$ \\
\hline Reflections collected & 8434 \\
\hline Independent reflections & $1809\left[R_{\text {int }}=0.0394, R_{\text {sigma }}=0.0173\right]$ \\
\hline Data/restraints/parameters & $1809 / 1 / 148$ \\
\hline Goodness-of-fit on $F^{2}$ & 1.053 \\
\hline Final $R$ indexes $[\mid>=2 \sigma(I)]$ & $R_{1}=0.0347, w R_{2}=0.0911$ \\
\hline Final R indexes [all data] & $\mathrm{R}_{1}=0.0347, w \mathrm{R}_{2}=0.0911$ \\
\hline Largest diff. peak/hole / e $\AA^{-3}$ & $0.29 /-0.41$ \\
\hline Flack parameter & $0.036(14)$ \\
\hline
\end{tabular}




\section{Crystal Data and Experimental (3a)}

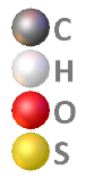

methyl (2S,5S)-5-(((S)-2-methoxypropanoyl)oxy)-1,3-

oxathiolane-2-carboxylate<smiles>COC(=O)C1O[C@@H](OC(=O)C(C)OC)CS1</smiles>

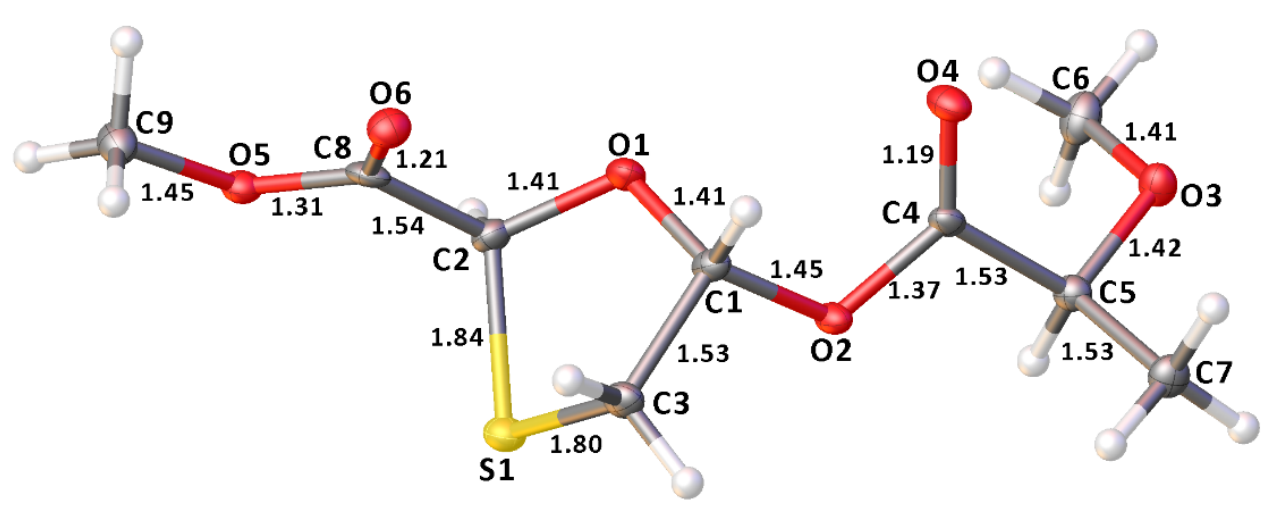

Figure S5: Depiction of 3a with data obtained from X-Ray analysis.

Sample of 3a was recrystallized from a toluene/hexanes mixture (1:1). A suitable crystal was selected and MiTeGen Microloop on a XtaLAB AFC11 (RINC): quarter-chi single diffractometer. The crystal was kept at 100.00(10) K during data collection. Using Olex2 [1], the structure was solved with the ShelXT [2] structure solution program using Intrinsic Phasing and refined with the olex2.refine [3] refinement package using Gauss-Newton minimisation. Crystallographic data and structural refinement parameters are given below. Crystallographic data has been deposited with the Cambridge Crystallographic Data Centre (CCDC) with the depository number: CCDC 1980800. This data is available from the CCDC via www.ccdc.cam.ac.uk/.

1. Dolomanov, O.V., Bourhis, L.J., Gildea, R.J, Howard, J.A.K. \& Puschmann, H. J. Appl. Cryst., 2009, 42, 339-341.

2. Sheldrick, G.M. Acta Cryst., 2015, A71, 3-8.

3. Bourhis, L.J., Dolomanov, O.V., Gildea, R.J., Howard, J.A.K., Puschmann, H. Acta Cryst., 2015, A71, 59-75.

\section{Crystal structure determination}

Crystal Data for $\mathrm{C}_{9} \mathrm{H}_{14} \mathrm{O}_{6} \mathrm{~S}(M=250.27 \mathrm{~g} / \mathrm{mol})$ : monoclinic, space group P2 ${ }_{1}$ (no. 4), $a=9.9207$ (3) $\AA$, $b=$ $5.4139(2) \AA, c=11.2533(3) \AA, b=106.958(3)^{\circ}, V=578.13(3) \AA^{3}, Z=2, T=100.00(10) \mathrm{K}, \mu(\mathrm{Cu} \mathrm{K \alpha})=2.634$ $\mathrm{mm}^{-1}$, Dcalc $=1.4376 \mathrm{~g} / \mathrm{cm}^{3}, 10630$ reflections measured $\left(8.22^{\circ} \leq 2 \Theta \leq 133.12^{\circ}\right), 1796$ unique $\left(R_{\text {int }}=\right.$ $\left.0.0632, R_{\text {sigma }}=0.0233\right)$ which were used in all calculations. The final $R_{1}$ was $0.0628(\mathrm{I}>=2 \mathrm{u}(\mathrm{I}))$ and $w R_{2}$ was 0.1716 (all data). 
Table S13: Select Crystal data and structure refinement

\begin{tabular}{|c|c|}
\hline Empirical formula & $\mathrm{C}_{9} \mathrm{H}_{14} \mathrm{O}_{6} \mathrm{~S}$ \\
\hline Formula weight & 250.27 \\
\hline Temperature/K & $100.00(10)$ \\
\hline Crystal system & monoclinic \\
\hline Space group & $\mathrm{P} 2_{1}$ \\
\hline $\mathrm{a} / \AA$ & $9.9207(3)$ \\
\hline $\mathrm{b} / \AA ̊ \AA$ & $5.4139(2)$ \\
\hline$c / \AA ̊$ & $11.2533(3)$ \\
\hline$\alpha /^{\circ}$ & 90 \\
\hline$\beta /^{\circ}$ & $106.958(3)$ \\
\hline$\gamma /^{\circ}$ & 90 \\
\hline Volume/ $/ \AA^{3}$ & $578.13(3)$ \\
\hline Z & 2 \\
\hline$\rho_{\text {calc }} g / \mathrm{cm}^{3}$ & 1.4376 \\
\hline$\mu / \mathrm{mm}^{-1}$ & 2.634 \\
\hline$F(000)$ & 265.6 \\
\hline Crystal size $/ \mathrm{mm}^{3}$ & $0.414 \times 0.243 \times 0.103$ \\
\hline Radiation & Cu K $\alpha(\lambda=1.54184)$ \\
\hline $2 \Theta$ range for data collection ${ }^{\circ}$ & 8.22 to 133.12 \\
\hline Index ranges & $-12 \leq h \leq 12,-5 \leq k \leq 6,-14 \leq \mathrm{I} \leq 13$ \\
\hline Reflections collected & 10630 \\
\hline Independent reflections & $1796\left[R_{\text {int }}=0.0632, R_{\text {sigma }}=0.0233\right]$ \\
\hline Data/restraints/parameters & $1796 / 1 / 148$ \\
\hline Goodness-of-fit on $F^{2}$ & 1.048 \\
\hline Final $R$ indexes $[\mid>=2 \sigma(I)]$ & $\mathrm{R}_{1}=0.0628, w \mathrm{R}_{2}=0.1716$ \\
\hline Final $R$ indexes [all data] & $\mathrm{R}_{1}=0.0628, w \mathrm{R}_{2}=0.1716$ \\
\hline Largest diff. peak/hole / e $\AA^{-3}$ & $0.72 /-0.43$ \\
\hline Flack parameter & $0.08(3)$ \\
\hline
\end{tabular}




\section{Cytosine Coupling to form 8}

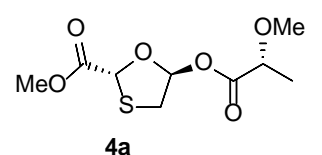

$4 a$

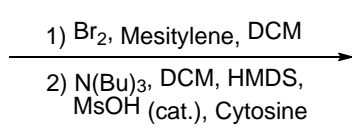

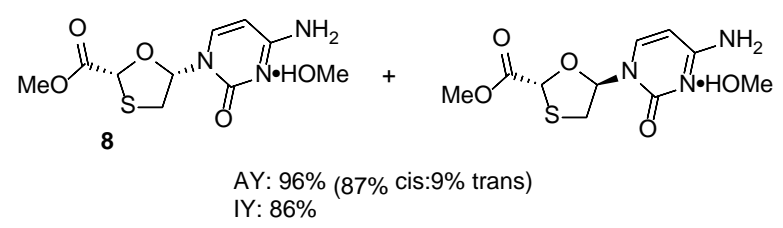

IY: $86 \%$

Table S14: Results of cytosine coupling from $100 \mathrm{mg}$ to $7.5 \mathrm{~g}$.

\begin{tabular}{|c|c|c|c|c|c|c|}
\hline Entry & Notes & Scale & Yield Cis & $\begin{array}{l}\text { Yield } \\
\text { Trans }\end{array}$ & $\begin{array}{l}\text { Yield } \\
\text { Total }\end{array}$ & Cis:Trans \\
\hline \multirow[t]{4}{*}{1} & Assay & $100 \mathrm{mg}$ & $83 \%$ & $10 \%$ & $92 \%$ & 8.68 \\
\hline & Crystallization & & $77 \%$ & $0 \%$ & & \\
\hline & Mother Liquour & & $8 \%$ & $7 \%$ & & \\
\hline & Total & & $85 \%$ & $7 \%$ & $92 \%$ & 12.1 \\
\hline \multirow[t]{3}{*}{2} & Crystallization & $250 \mathrm{mg}$ & $79 \%$ & $0 \%$ & & \\
\hline & Mother Liquour & & $11 \%$ & $5 \%$ & & \\
\hline & Total & & $90 \%$ & $5 \%$ & $94 \%$ & 19.0 \\
\hline \multirow[t]{4}{*}{3} & Assay-3hr & & $83 \%$ & $7 \%$ & $90 \%$ & 12.2 \\
\hline & Crystallization & $250 \mathrm{mg}$ & $80 \%$ & $0 \%$ & & \\
\hline & Mother Liquour & & $4 \%$ & $6 \%$ & & \\
\hline & Total & & $84 \%$ & $6 \%$ & $91 \%$ & 13.1 \\
\hline \multirow[t]{3}{*}{4} & Assay-3hr & & $87 \%$ & $9 \%$ & $96 \%$ & 9.67 \\
\hline & Crystallization & $7.5 \mathrm{~g}$ & $86 \%$ & $0 \%$ & & \\
\hline & Total & & $86 \%$ & $0 \%$ & $86 \%$ & \\
\hline
\end{tabular}

\section{Typical Procedure for Synthesis of Cytosinyl Oxathiolane 8:}

Cytosine (4.94 g, $44.5 \mathrm{mmol}, 1.5$ equiv., >98\% TCl Chemicals), hexamethyldisilazane (10.6 mL, 50.4 mmol, 1.70 equiv., 99.9\% Sigma-Aldrich), methanesulfonic acid (19.3 $\mu \mathrm{L}, 0.297 \mathrm{mmol}, 1 \mathrm{~mol} \%,>99 \%$ Sigma-Aldrich), and toluene ( $12 \mathrm{~mL},>99.5 \%$ MilliporeSigma) were loaded into a dried $250 \mathrm{~mL}$ Schlenk flask equipped with a condenser and under argon atmosphere. The flask was heated to reflux at $135^{\circ} \mathrm{C}$ until all cytosine went into solution, approximately $60 \mathrm{~min}$. The reaction was judged to be complete as ammonia production ceased. A stream of nitrogen was passed through the vessel for 20 min to remove excess HMDS. The silylated cytosine solution was removed from heat and while hot, dichloromethane (40 mL, 99.8\%, MilliporeSigma) was slowly added and cooled the reaction via reflux. After addition of dichloromethane, tributylamine was added $(10.6 \mathrm{~mL}, 44.5 \mathrm{mmol}, 1.5$ equiv., $>98.5 \%$ Sigma-Aldrich). The bromooxathiolane was prepared simultaneously in a separate reaction flask. Oxathiolane $4 \mathrm{a}$ ( $7.50 \mathrm{~g}, 99$ wt\%, $29.7 \mathrm{mmol}$, 1 equiv.), mesitylene (8.25 mL, $59.3 \mathrm{mmol}, 2$ equiv., >98\% Sigma-Aldrich), and dichloromethane $(15 \mathrm{~mL}$ ) were loaded into a dried $100 \mathrm{~mL}$ Schlenk flask under argon atmosphere. The solution was cooled to $-15^{\circ} \mathrm{C}$ with a $\mathrm{NaCl} /$ ice bath. At this point, flow of argon was stopped and stirrate 
was set at $100 \mathrm{RPM}$ to encourage dissolution of $\mathrm{HBr}$ in dichloromethane. Bromine $(2.28 \mathrm{~mL}, 44.5 \mathrm{mmol}$, 1.5 equiv., reagent grade Sigma-Aldrich) was dissolved in dichloromethane $(20 \mathrm{~mL})$ and added to oxathiolane over the course of $3 \mathrm{~min}$. The solution was held at $-15^{\circ} \mathrm{C}$ for $30 \mathrm{~min}$ and monitored by NMR for consumption of starting material. Bromooxathiolane was produced quantitatively. The reaction was warmed to room temperature, the stir-rate was increased to 400 RPM, and the solution was sparged with argon to remove residual $\mathrm{HBr}$. The argon stream was positioned below the liquid level surface with gas bubbling through the solution. Sparging stopped after 20 minutes, at which point, vapors of $\mathrm{HBr}$ were no longer observed. The cytosine solution was cooled with a $\mathrm{NaCl} /$ ice bath to $-15{ }^{\circ} \mathrm{C}$, and bromooxathiolane was added to the cytosine via cannula transfer over the course of 10-15 min. Importantly, at the time transfer begins all cytosine remains in solution and has not crystallized out of solution. At the end of addition, a light yellow homogeneous solution is obtained. The reaction is kept at $-15^{\circ} \mathrm{C}$ for $1 \mathrm{hr}$ before warming to room temperature. The reaction is stirred at room temperature for $8 \mathrm{hr}$. A light stream of argon is passed through the reaction vessel for the duration of the experiment to keep the solution homogeneous. Otherwise, a suspension is obtained, presumably from hydrolysis of a silyl group. After stirring at room temperature for $8 \mathrm{hr}$, solvent is removed by vacuum to strip off any trimethylsilyl bromide generated during nucleoside formation. A clear oil to semi-viscous suspension is obtained. Methanol ( $60 \mathrm{~mL},>99.8 \%$ BDH Chemicals) is added and heated to reflux for 5 min bringing all material into solution. During heating, small sugar-like crystals of $\mathbf{8}$ begin to form, which are a complex of nucleoside and methanol. The reaction is cooled to room temperature and held for $2 \mathrm{hr}$. The reaction is further cooled at $-15{ }^{\circ} \mathrm{C}$ for $12 \mathrm{hr}$. The solids are collected via vacuum filtration and washed with two portions of room temperature methanol $(5 \mathrm{~mL})$. Solids are dried and analyzed for purity $(8.524$ $\mathrm{g}, 77 \mathrm{wt} \%, 86 \%$ yield). To increase purity and remove residual cytosine, $7.33 \mathrm{~g}$ of the solids ( $77 \%$ purity, $22.0 \mathrm{mmol}$ ) are mixed with methanol and heated to reflux for $1 \mathrm{hr}$. The solution is cooled to room temperature and let stand for $2 \mathrm{hr}$. The mixture is then cooled to $-15^{\circ} \mathrm{C}$ for $12 \mathrm{hr}$ and the solids are collected by vacuum filtration and washed twice with methanol. Solids are dried and analyzed for purity (6.18 g 8, 91.2\% purity, 88.9\% Max Theoretical for $\mathrm{MeOH}$ adduct), $21.9 \mathrm{mmol}, 99.5 \%$ recovery). ${ }^{1} \mathrm{H}$ NMR (600 MHz, DMSO) $\delta 7.94$ (d, $J=7.5 \mathrm{~Hz}, 1 \mathrm{H}), 7.30(\mathrm{bs}, 2 \mathrm{H}), 6.35(\mathrm{~m}, 1 \mathrm{H}), 5.80(\mathrm{~d}, J=7.5 \mathrm{~Hz}, 1 \mathrm{H}), 5.73(\mathrm{~s}, 1 \mathrm{H}), 4.10$ (q, $J=5.3 \mathrm{~Hz}, 1 \mathrm{H}), 3.75(\mathrm{~s}, 3 \mathrm{H}), 3.53(\mathrm{dd}, J=11.9,5.0 \mathrm{~Hz}, 1 \mathrm{H}), 3.17(\mathrm{~d}, J=4.3 \mathrm{~Hz}), 3.13(\mathrm{dd}, J=11.9,6.7 \mathrm{~Hz}, 1 \mathrm{H}) .{ }^{13} \mathrm{C}$ NMR (151 MHz, DMSO) $\delta 170.40$ (s), 166.17 (s), 155.21 (s), 141.02 (s), 94.99 (s), 89.13 (s), 77.60 (s), 53.22 (s), 49.06 (s), 35.71 (s). HRMS: Expected M+H m/z 258.0548, Found: 258.0540.

\section{Reaction Sensitivities-Important Notes for Reproduction:}

- Reaction must be kept under strictly inert atmosphere. Failure to do so leads to of hydrolysis of silyl groups (nucleobase, nucleoside, TMSBr). The reaction will become heterogeneous either at the cytosine silylation stage or at the nucleophilic addition.

- Dilute bromine in dichloromethane. Reaction of bromine and mesitylene is exothermic and addition of bromine neat creates hotspots which lead to loss of gaseous $\mathrm{HBr}$.

- Reaction kept slightly pressurized during addition of bromine to mesitylene and oxathiolane. This is done in order to favor volatile $\mathrm{HBr}$ remaining in solution. The stirrate is kept low for similar reasons.

- Sparge bromooxathiolane solution at end of reaction to remove excess $\mathrm{HBr}$. This is done to prevent $\mathrm{HBr}$ from cleaving silyl groups, but more importantly to facilitate purification after addition of cytosine. The protonation state appears to be important to fully recover all product 
in the crystallization. We had more success crystallizing the neutral form than the protonated form.

- Remove excess HMDS from silylation solution through sparge with inert gas or vacuum.

Presence of alternative nucleophiles appears to lower yield of nucleoside.

- Add bromooxathiolane to silylated cytosine. The silyl group on the amine is easily hydrolyzed and when that occurs, an alternate nucleophile is present. In a cytosine rich, bromooxathiolane deficient solution, this is less of a problem than in a bromooxathiolane rich environment. Best yields were obtained when adding bromooxathiolane to cytosine.

- Add bromooxathiolane while silylated cytosine is full solvated. If silylated cytosine is cooled and let stand at low temperature for an extended time it will crystallize from solution. Adding bromooxathiolane under these conditions is similar to adding cytosine to a solution of bromooxathiolane which is not preferred for the reasons described above. Best yields were obtained when adding to a fully homogeneous solution of silylated cytosine.

- Concentrate reaction mixture at end of reaction to remove any TMSBr formed during coupling. Presence of $\mathrm{TMSBr}$ can interfere with crystallization by producing $\mathrm{HBr}$ in quench with methanol.

- After methanol addition, cool at room temperature for at least 2 hours. Cooling too quickly leads to much higher levels of impurities in the product and also decrease yield by interfering with the crystallization. 
methanol adduct of methyl (2R,5S)-5-(4-amino-2-oxopyrimidin$1(2 H)$-yl)-1,3-oxathiolane-2-carboxylate<smiles></smiles>

${ }^{1} \mathrm{HNMR}(600 \mathrm{MHz}, \mathrm{DMSO}) \delta 7.94(\mathrm{~d}, J=7.5 \mathrm{~Hz}, 1 \mathrm{H}), 7.30(\mathrm{bs}, 2 \mathrm{H}), 6.35(\mathrm{~m}, 1 \mathrm{H}), 5.80(\mathrm{~d}, J=7.5 \mathrm{~Hz}, 1 \mathrm{H}), 5.73(\mathrm{~s}, 1 \mathrm{H}), 4.10(\mathrm{q}, J=5.3 \mathrm{~Hz}, 1 \mathrm{H}), 3.75(\mathrm{~s}, 3 \mathrm{H}), 3.53(\mathrm{dd}, J=11.9,5.0$ $\mathrm{Hz}, 1 \mathrm{H}), 3.17(\mathrm{~d}, J=4.3 \mathrm{~Hz}), 3.13(\mathrm{dd}, J=11.9,6.7 \mathrm{~Hz}, 1 \mathrm{H})$.

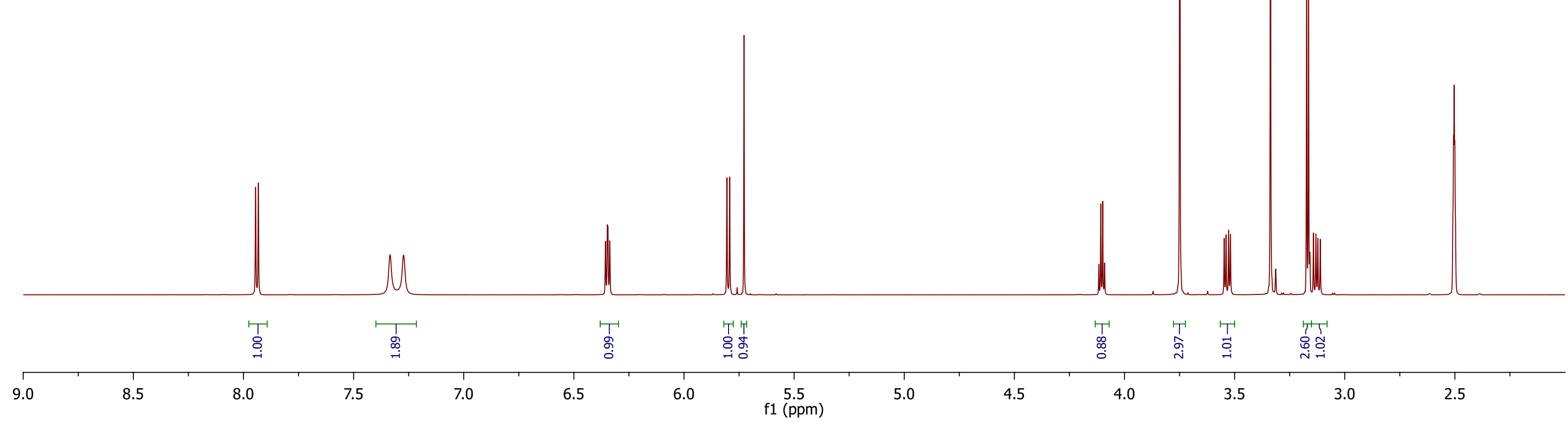


methanol adduct of

methyl (2R,5S)-5-(4-amino-2-oxopyrimidin-

$1(2 H)$-yl)-1,3-oxathiolane-2-carboxylate

$\mathrm{MeO}_{\mathrm{S}}^{\mathrm{O}}{ }_{\mathrm{O}}$ 
In Process Control: Quantitative Conversion to

Bromo-oxathiolane Referenced Against MeCN

${ }_{\mathrm{MeO}}^{\mathrm{O}} \mathrm{S}_{\mathrm{S}}^{\mathrm{O}} \gamma_{90 \%}^{\mathrm{Br}}+\mathrm{MeO}_{\mathrm{S}}^{\mathrm{O}}>_{10 \%}^{\mathrm{O}}$

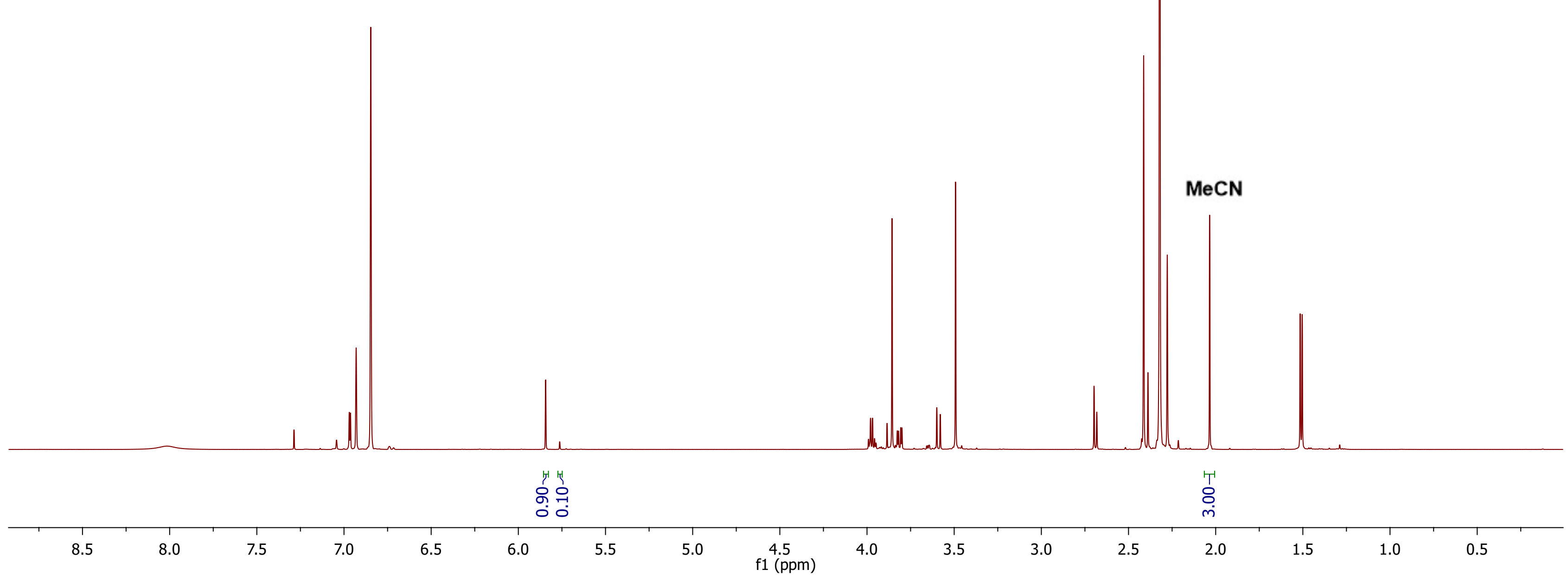




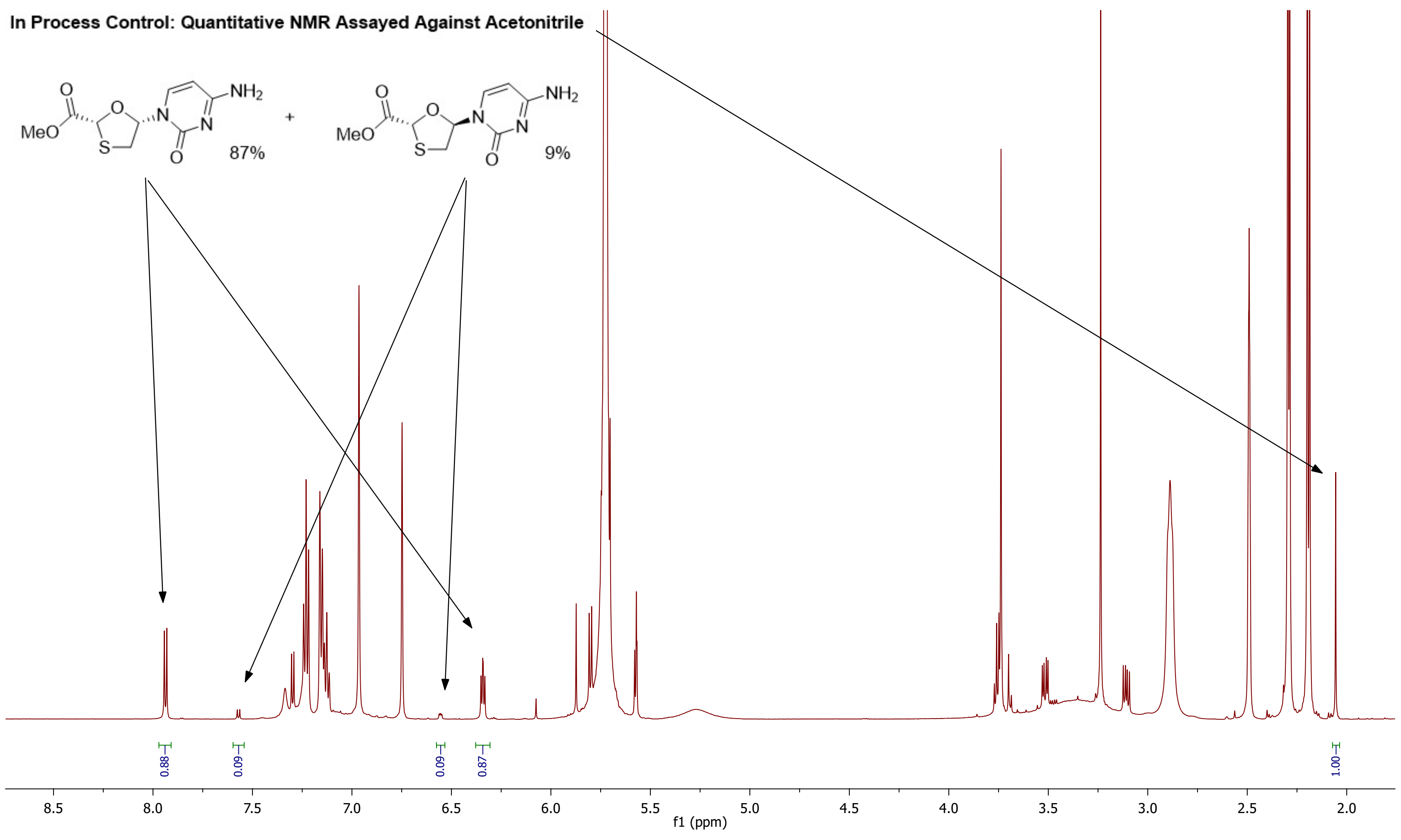


Sample Crystallized from Reaction Mixture

Sample Assayed for Purity by Quantitative NMR vs MeCN: $77 \%$

$\mathrm{MeO}_{\mathrm{S}}^{\mathrm{O}} \overbrace{\mathrm{O}}^{\mathrm{O}}$

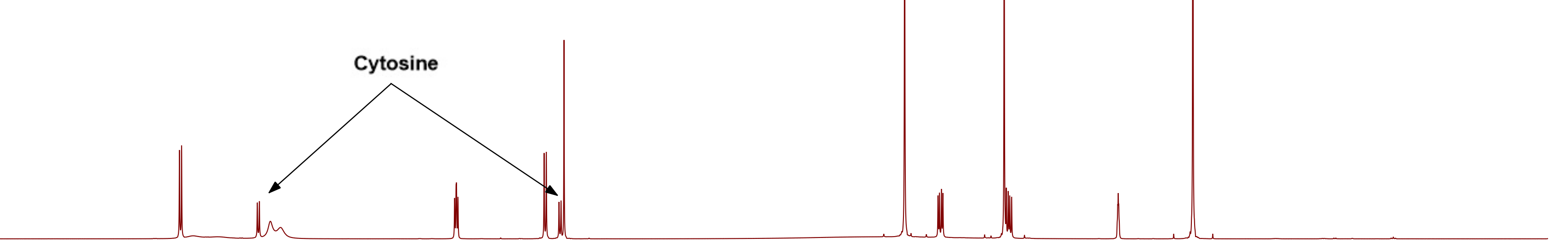

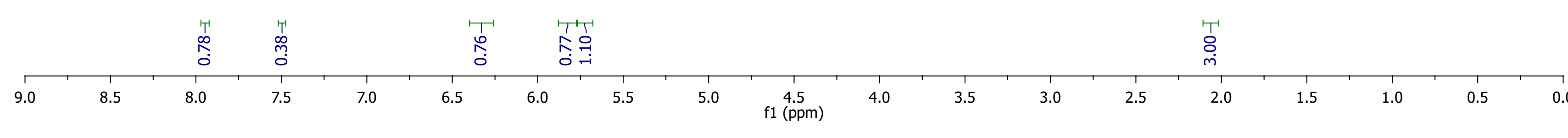


MeOH Crystallized Sample with Partial Complexation of $\mathrm{MeOH}$

Sample Assayed for Purity by Quantitative NMR vs MeCN: $91.2 \%$ Theoretical Max for MeOH Adduct: $\mathbf{8 8 . 9 \%}$<smiles></smiles>

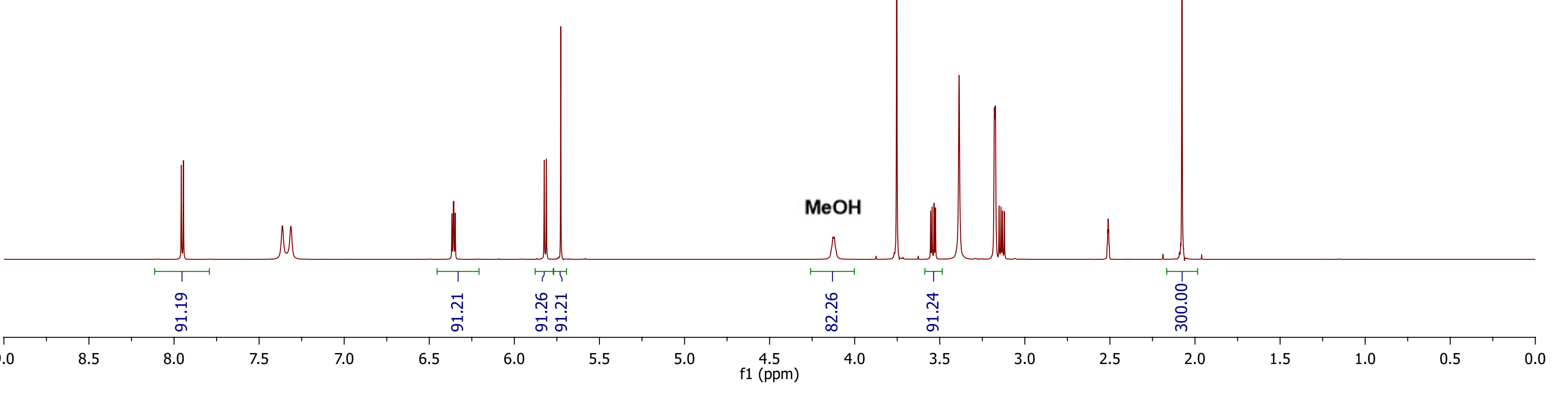




\section{SFC Analysis Conditions:}

Column: ChiralPak IA, $4.6 \mathrm{~mm}$ ID X $250 \mathrm{mmL}, 3 \mathrm{uM}$ particles Mobile Phase: $20 \%$ Methanol (0.5\% DEA) in $\mathrm{CO}_{2}$

Flow Rate: $2.0 \mathrm{~mL} / \mathrm{min}$

Detection: $275 \mathrm{~nm}$

\section{Retention Times:}

Cytosine

8 (2R, 5S-desired)

8 (2S, 5R-enantiomer)

$8(2 S, 5 S$ and $2 R, 5 R$-trans diastereomers)
$5.2 \mathrm{~min}$

$5.4 \mathrm{~min}$

$6.5 \mathrm{~min}$

7.0 and $7.7 \mathrm{~min}$ 
Sample made from mixture of trans-acetate:

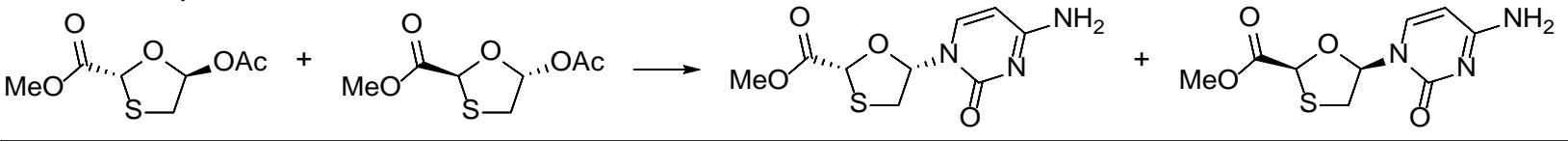

DAD1 B, Sig=275,4 Ref=360,100 (C:IUSERSIP...1292013TC 2020-01-29 08-59-49I001-D1F-B2-ia340-Penultimate.D)

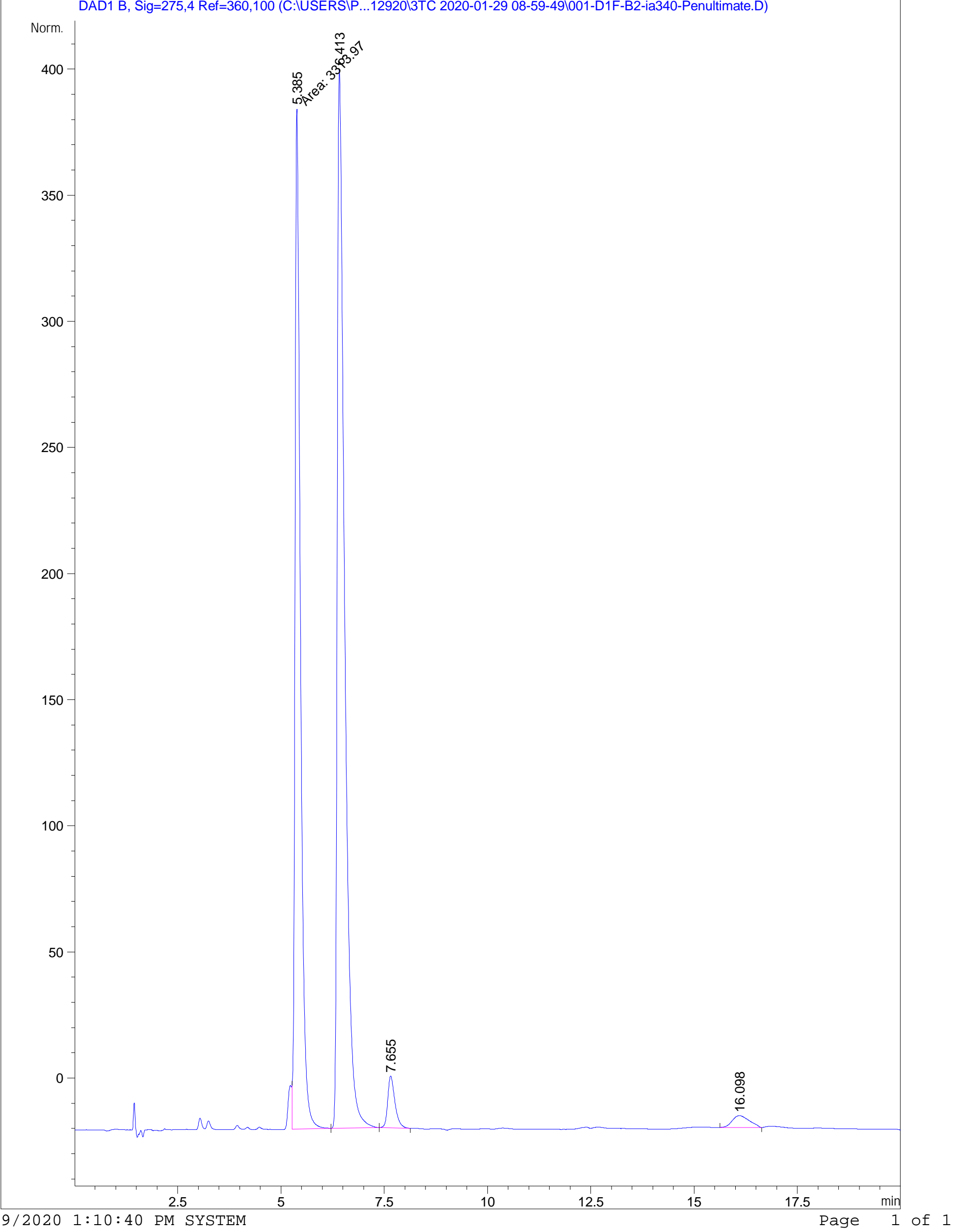


Sample made from mixture of trans-acetate:

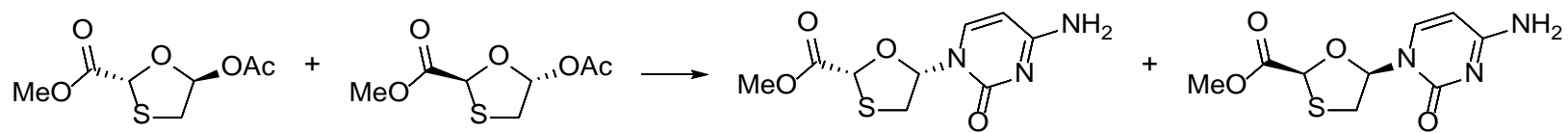

\section{SFC Report}

Data file:

Sample name:

Description:

Sample amount:

Instrument:

Injection date:

Acq. method:

Analysis method:

Last changed:

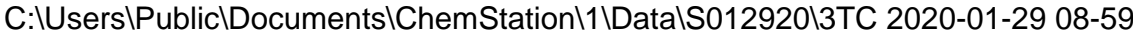
-49\001-D1F-B2-ia340-Penultimate.D ia340-Penultimate

Old Sample

0.000

Sample type:

Sample

SFC

1/29/2020 9:00:42 AM

Location:

Injection:

D1F-B2

3TC Chiral.M

3TC Chiral.M

1/31/2020 7:16:21 AM

Acq. operator:

5.000

SYSTEM
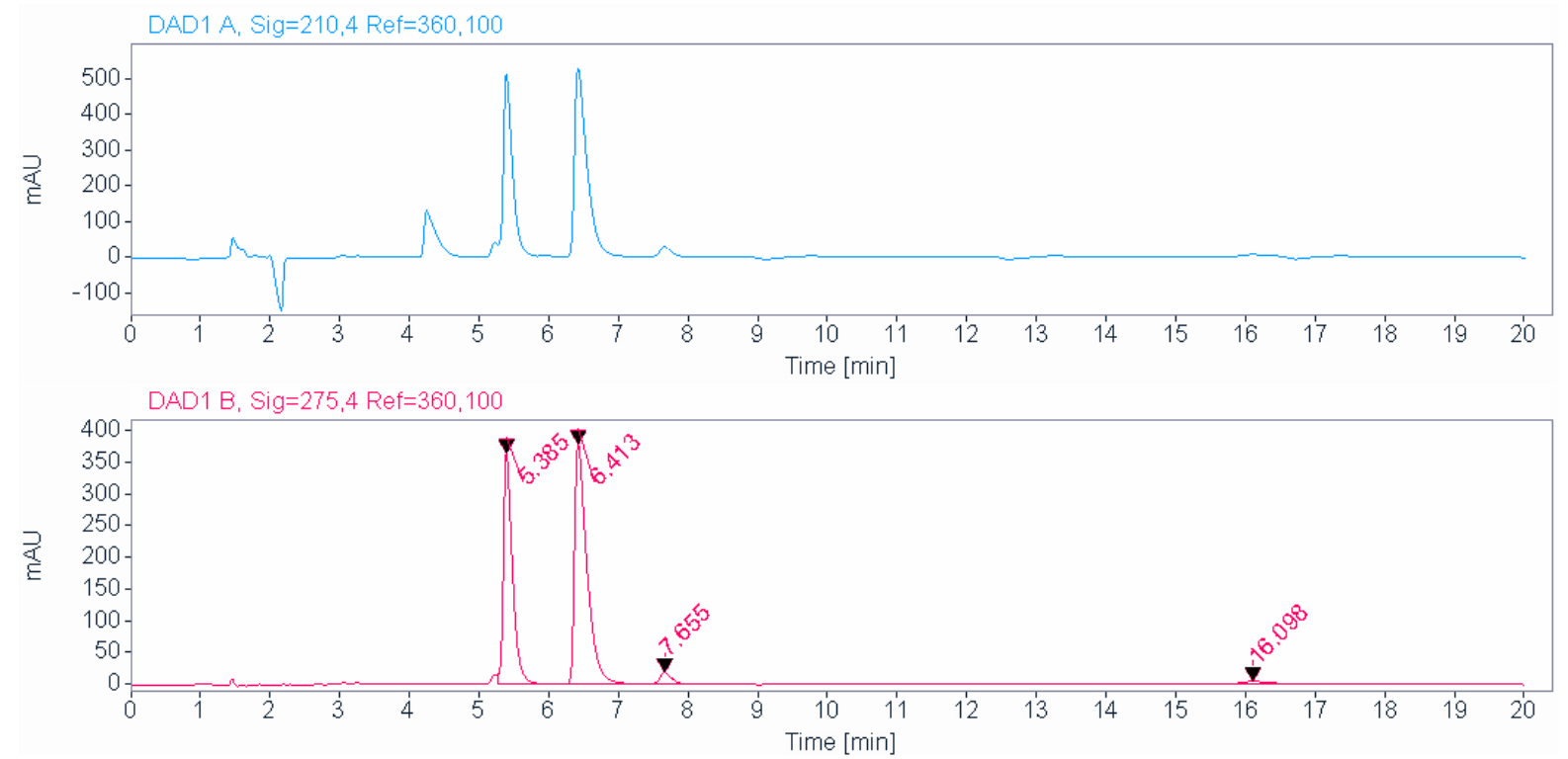

Signal: $\quad$ DAD1 B, Sig $=275,4$ Ref $=360,100$

$\begin{array}{rrrrr}\text { RT [min] Type } & \text { Width [min] } & \text { Area } & \text { Height } & \text { Area\% Name } \\ 5.385 \text { FM } & 0.1514 & 3313.9656 & 364.8784 & 39.6682 \\ 6.413 \text { BB } & 0.1826 & 4693.5972 & 378.5927 & 56.1824 \\ 7.655 \text { BB } & 0.1835 & 224.5148 & 18.5114 & 2.6874 \\ 16.098 \text { BB } & 0.4222 & 122.1317 & 4.2630 & 1.4619 \\ & \text { Sum } & 8354.2092 & & \end{array}$


Reaction Mixture from Cytosine Coupling:

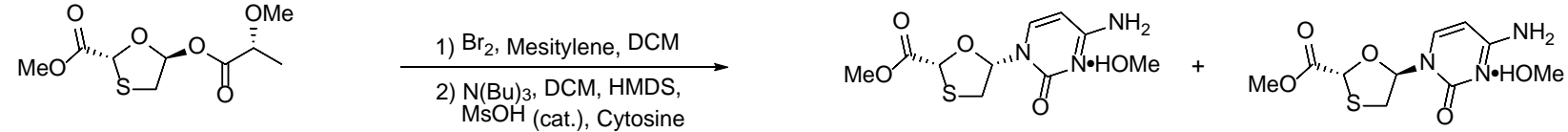

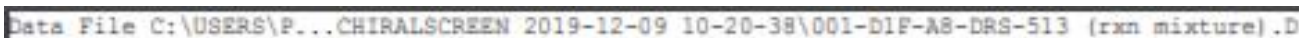

Sample Name: DRS-513 (rxan nixture)

$\begin{array}{lrl}\text { Acq. Operator : SYSTEM } & \text { Seq. Line : } & 1 \\ \text { Sample Operator : SYSTEM } & \text { Location : } & \text { DIF-h8 } \\ \text { Acq. Instrunent : SFC } & \text { Inj : } 1 \\ \text { Injection Date : } 12 / 9 / 201910: 21: 28 \text { AM } & \text { Inj Volune : } 5.000 \text { pI }\end{array}$

Different Inj Volume from Sample Entry! Actual Inj Volume : $1.000 \mu 1$

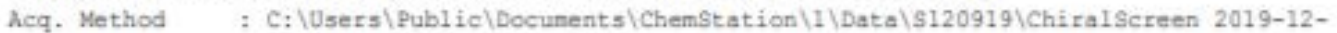
09 10-20-38)3TC Chiral, M

Last changed : 12/3/2019 9:57:31 AM by SYSzEM

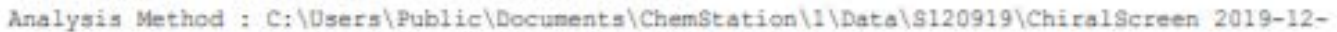

09 10-20-38) $3 T C$ Chiral.M (Sequence Method)

Last changed : $12 / 9 / 201912: 48: 43$ PM by SYSTEM

Nethod Info : IA Column- $25 \mathrm{~cm} \times 4.5 \mathrm{~mm}, 3 \mathrm{uMz} 20+$ Methanol/0.5h DEA, $2.0 \mathrm{~mL} / \mathrm{min}, 25 \mathrm{C}, 5$ uL.

Additional Info : Peak (s) manually integrated

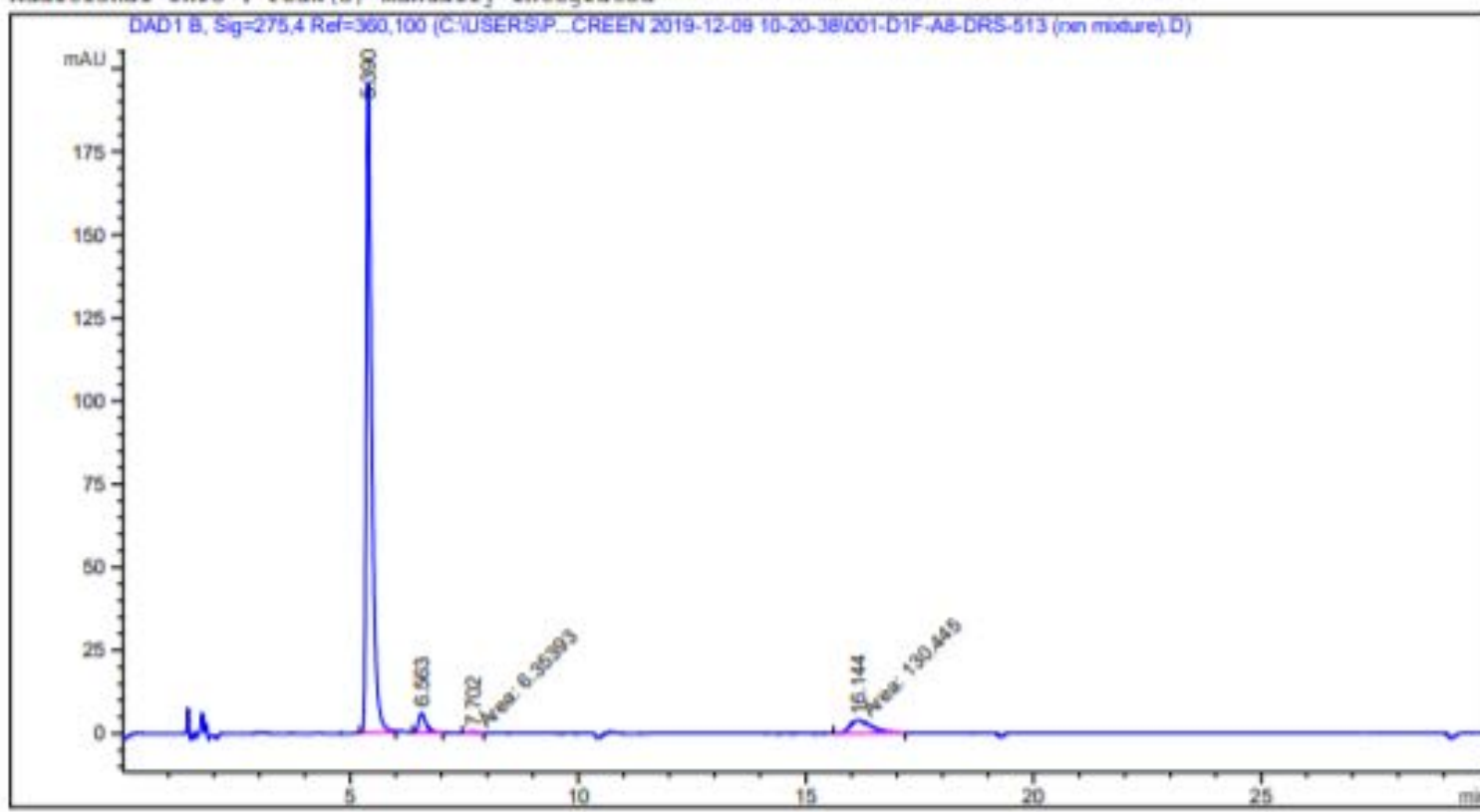

Area Percent Repart

$\begin{array}{lll}\text { Sorted By } & : & \text { Signal } \\ \text { Multiplier } & ; & 1.0000 \\ \text { Dilution } & ; & 1.0000\end{array}$

Use Multiplier 6 Dilution Factor with ISTDs

Signal 1: DAD1 B, Sig $=275,4$ Ref $=360,100$ 


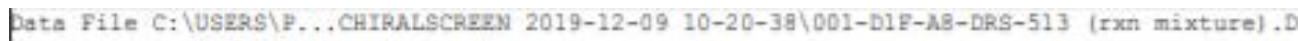
Sample Nane: DRS-513 (rxn nixture)

\begin{tabular}{|c|c|c|c|c|c|c|}
\hline $\begin{array}{c}\text { Peak } \\
\text { f }\end{array}$ & $\begin{array}{c}\text { RetTime } \\
\text { [min] }\end{array}$ & Type & $\begin{array}{l}\text { Width } \\
\text { [min] }\end{array}$ & $\begin{array}{c}\text { Area } \\
\text { [ndes] }\end{array}$ & $\begin{array}{l}\text { Height } \\
\text { [mlu] }\end{array}$ & $\begin{array}{c}\text { Area } \\
3\end{array}$ \\
\hline-1 & $1-2 .+2$ & 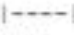 & $\ldots+\cdots$ & 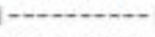 & 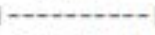 & 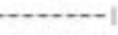 \\
\hline 1 & 5.390 & PB & $0.126 \mathrm{a}$ & 1658.37915 & 195.86514 & 89.1514 \\
\hline 2 & 6.563 & BE & 0.1676 & 55.00482 & 5.85205 & 3.4945 \\
\hline 3 & 7.702 & MA4 & 0.2337 & 6.35393 & $4.53218 e-1$ & 0.3416 \\
\hline 4 & 16.144 & DEA & 0.5613 & 130.44539 & 3.87309 & 7.0125 \\
\hline & Is : & & & 1860.18329 & 206.04350 & \\
\hline
\end{tabular}

$\cdots$ End of Report $\cdots$ 


\section{Product crystallized from cytosine coupling reaction mixture:}<smiles>COC(=O)C1OC(OC(=O)C(C)C(=O)OC)CS1</smiles>

\begin{tabular}{c} 
1) $\mathrm{Br}_{2}$, Mesitylene, DCM \\
\hline 2) $\mathrm{N}(\mathrm{Bu})_{3}, \mathrm{DCM}, \mathrm{HMDS}$, \\
$\mathrm{MSOH}$ (cat.), Cytosine
\end{tabular}

$\mathrm{MeO}_{\mathrm{S}}{ }_{\mathrm{O}}^{\mathrm{O}}{ }_{\mathrm{O}}^{\mathrm{O}}$<smiles></smiles>
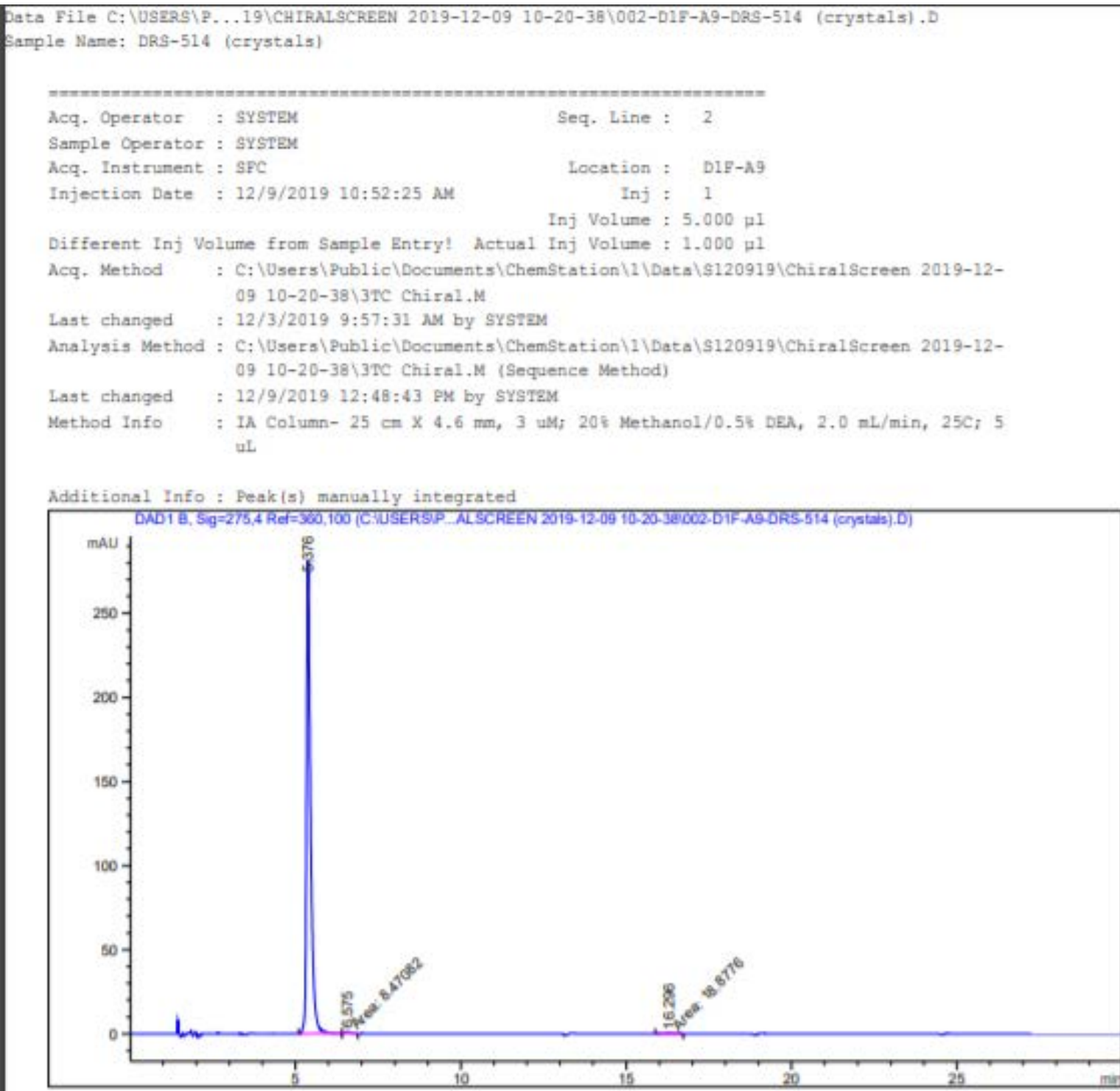


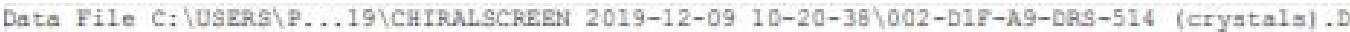
Sample Name: DRS-514 (crystals)

\begin{tabular}{|c|c|c|c|c|c|c|}
\hline $\begin{array}{c}\text { Peak } \\
\text {; }\end{array}$ & $\begin{array}{c}\text { RetTime } \\
\text { [min] }\end{array}$ & Type & $\begin{array}{l}\text { Width } \\
\text { [min] }\end{array}$ & $\begin{array}{c}\text { Area } \\
{\left[n M D^{*} s\right]}\end{array}$ & $\begin{array}{l}\text { Beight } \\
\text { [mXl] }\end{array}$ & $\begin{array}{c}\text { Area } \\
\&\end{array}$ \\
\hline & & & & $-2+\cos (2)-1$ & 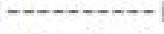 & 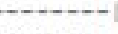 \\
\hline 1 & 5.376 & BA & 0.1302 & 2468.98462 & 281.71082 & 98.9045 \\
\hline 2 & 6.575 & Mat & 0.1728 & 8.47082 & E. $1721 \mathrm{De}-1$ & 0.3393 \\
\hline 3 & 16.296 & IN4 & 0.5723 & 18,87759 & $5.49718 \mathrm{e}-1$ & 0.7562 \\
\hline Fotal & s : & & & 2496,33303 & 283.07774 & \\
\hline
\end{tabular}

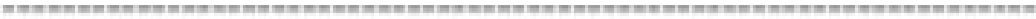

$\cdots$ End of Report $\cdots$ 


\section{Recrystallized Product}

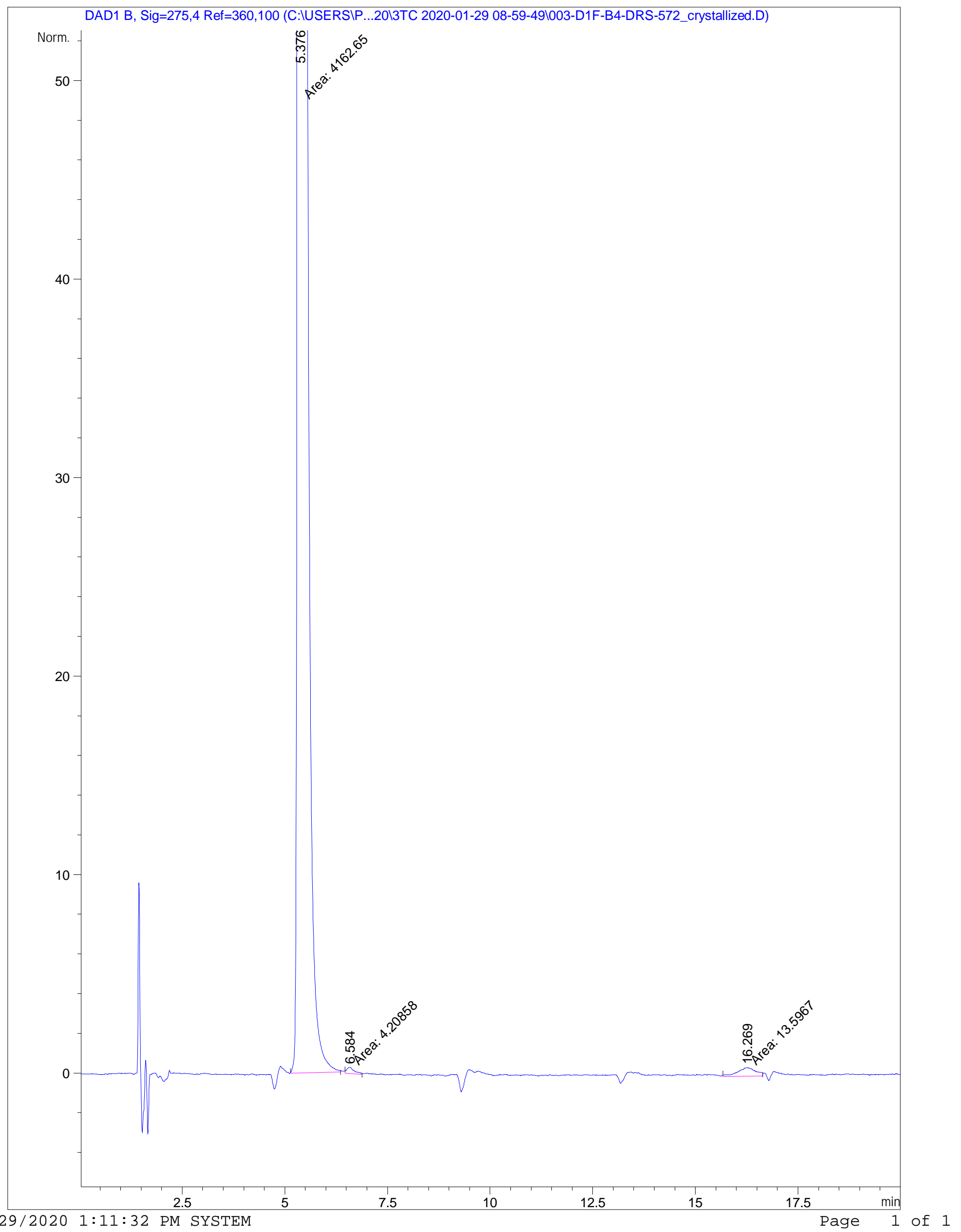




\section{Recrystallized Product}<smiles>COC(=O)C1OC(OC(=O)C(C)C)CS1</smiles>

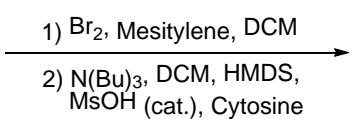

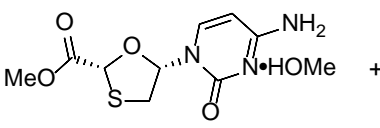

$\mathrm{MeO}_{\mathrm{S}} \mathrm{I}_{\mathrm{O}}^{\mathrm{O}}$

\section{SFC Report}

Data file:

Sample name:

Description:

Sample amount:

Instrument:

Injection date:

Acq. method:

Analysis method:

Last changed:
C: $\mid$ Users $\backslash$ Public $\backslash D o c u m e n t s \backslash C h e m S t a t i o n \backslash 1 \backslash D a t a \backslash S 012920 \backslash 3 T C ~ 2020-01-29 ~ 08-59$ -49\003-D1F-B4-DRS-572_crystallized.D DRS-572_crystallized

Penultimate

0.000

SFC

1/29/2020 9:42:33 AM

3TC Chiral.M

3TC Chiral.M

1/31/2020 7:16:21 AM

(modified after loading)
Sample type: Sample

\section{Location: D1F-B4}

Injection: $\quad 1$ of 1

Injection volume: $\quad 5.000$

Acq. operator: SYSTEM
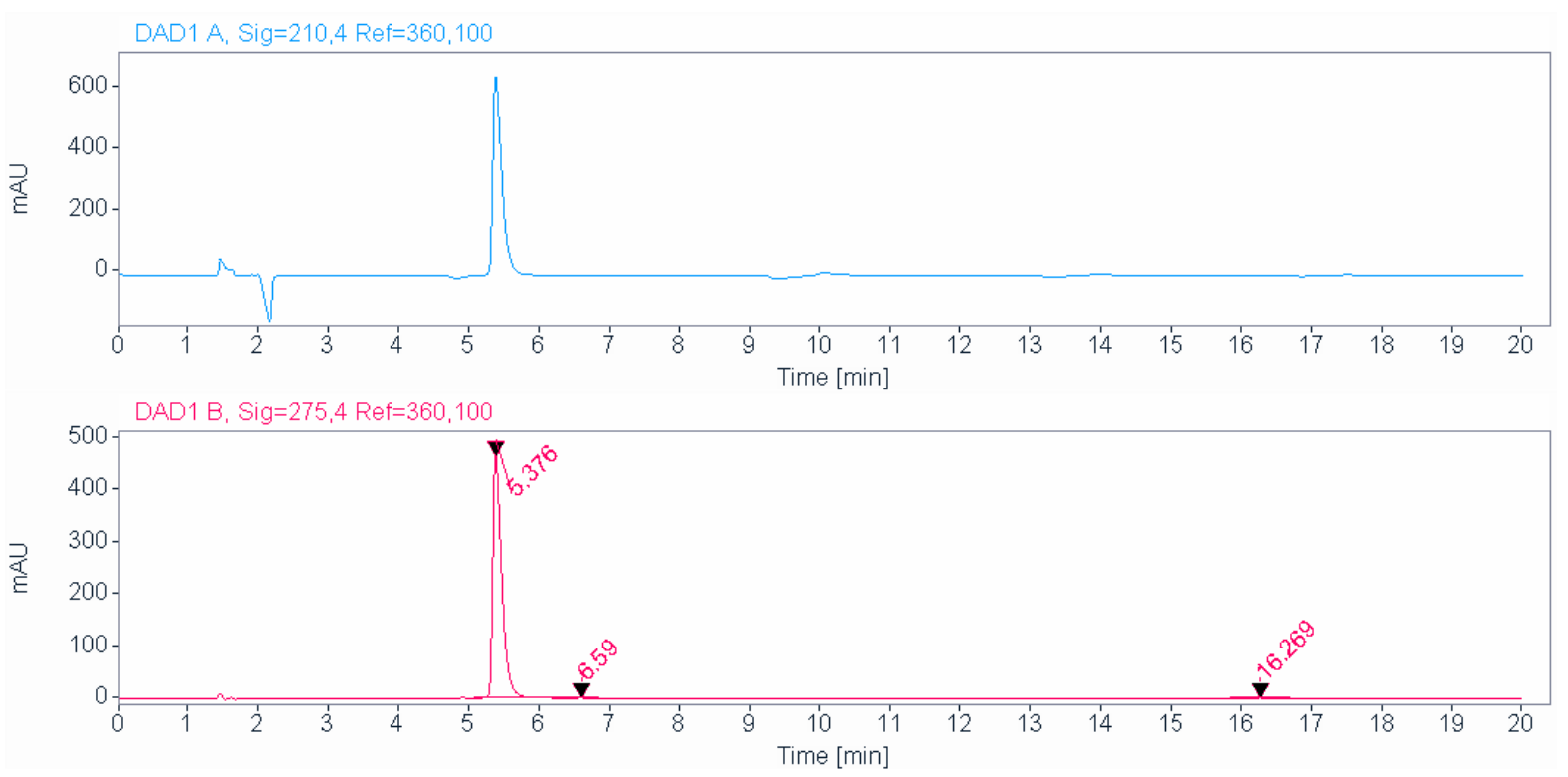

Signal: $\quad$ DAD1 B, Sig $=275,4$ Ref $=360,100$

$\begin{array}{rrrrc}\text { RT [min] Type } & \text { Width [min] } & \text { Area } & \text { Height } & \text { Area\% Name } \\ 5.376 \mathrm{MM} & 0.1492 & 4161.4937 & 464.9450 & 99.7390 \\ 6.590 \mathrm{MM} & 0.1624 & 2.1943 & 0.2252 & 0.0526 \\ 16.269 \mathrm{MM} & 0.4197 & 8.6936 & \text { ก.245? } & 0.2084 \\ & \text { Sum } & 4172.3815 & & \end{array}$




\section{Crystal Data and Experimental}

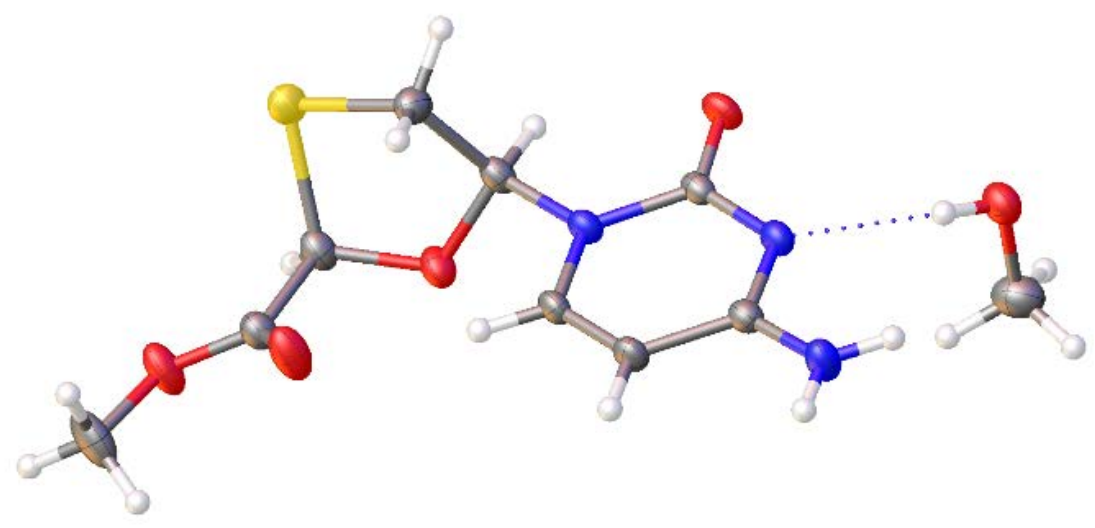

Figure S6: Depiction of $\mathbf{8}$ with data obtained from X-Ray analysis.

Hydrogen bond network shown below:

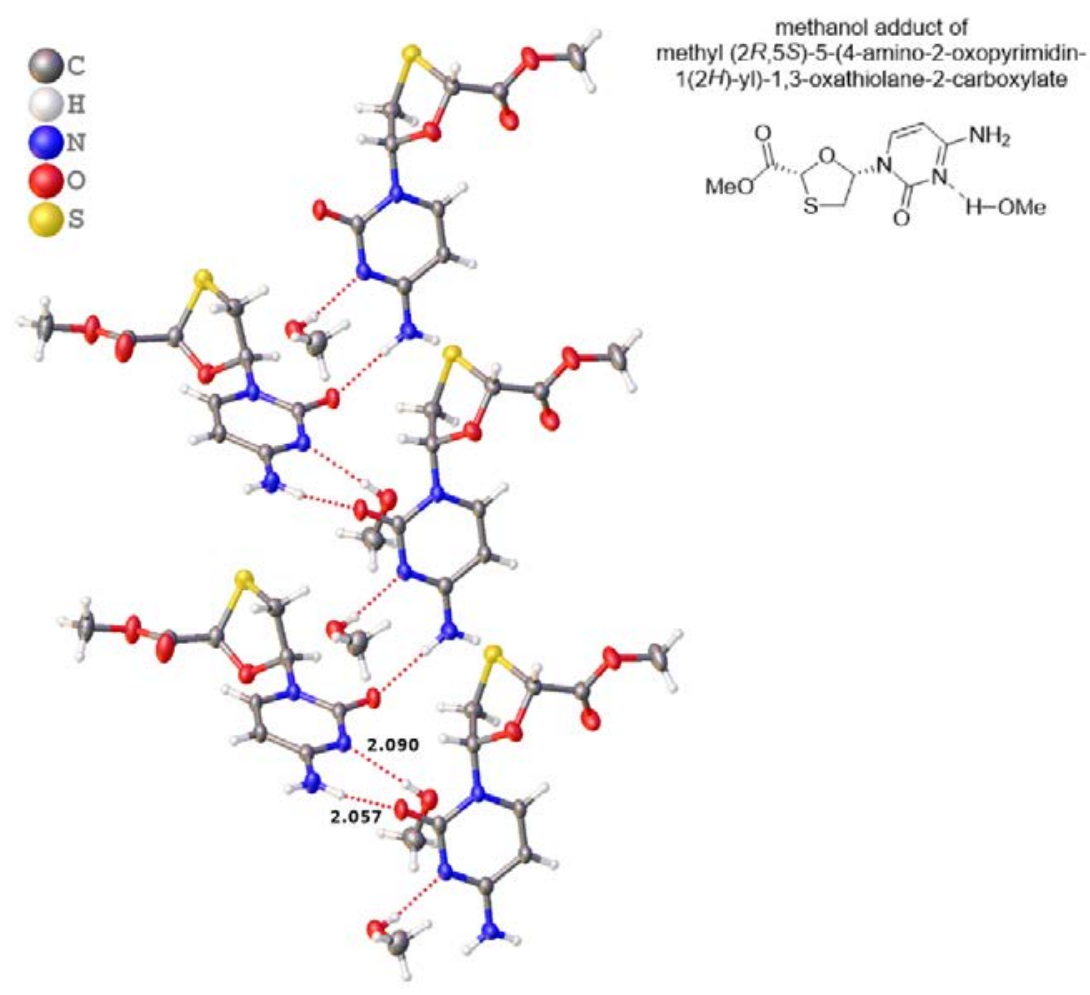

Figure S7: Depiction of 8 with data obtained from X-Ray analysis. 
Experimental. Crystals were obtained by dissolving $0.100 \mathrm{~g}$ of sample in $3.5 \mathrm{~mL}$ of refluxing methanol and cooling at room temperature. A suitable crystal was selected and mounted on a suitable support on a MiTeGen Microloop on a XtaLAB AFC11 (RINC): quarter-chi single diffractometer. The crystal was kept at a steady $T=293(2) \mathrm{K}$ during data collection. The structure was solved with the ShelXT 2014/4 (Sheldrick, 2014) structure solution program using the Intrinsic Phasing solution method. The model was refined with version 2016/6 of ShelXL-2016/6 (Sheldrick, 2016) using Least Squares 66inimization. Crystallographic data and structural refinement parameters are given below. Crystallographic data has been deposited with the Cambridge Crystallographic Data Centre (CCDC) with the depository number: CCDC 1980805. This data is available from the CCDC via www.ccdc.cam.ac.uk/.

Crystal Data. $\mathrm{C}_{10} \mathrm{H}_{15} \mathrm{~N}_{3} \mathrm{O}_{5} \mathrm{~S}, M_{r}=289.31$, monoclinic, $P 2_{1}$ (No. 4), $\mathrm{a}=6.9658(2) \AA, b=8.8494(2) \AA, c=$ $10.8590(3) \AA, b=105.295(3)^{\circ}, a=g=90^{\circ}, V=645.67(3) \AA^{3}, T=293(2) \mathrm{K}, Z=2, Z^{\prime}=1, m\left(\mathrm{CuK}_{a}\right)=2.453,2890$ reflections measured, 1576 unique $\left(R_{\text {int }}=0.0158\right)$ which were used in all calculations. The final $w R_{2}$ was 0.0683 (all data) and $R_{1}$ was $0.0251(\mathrm{I}>2(\mathrm{I}))$. 
Table S15: Select Crystal data and structure refinement

\begin{tabular}{|c|c|}
\hline Formula & $\mathrm{C}_{10} \mathrm{H}_{15} \mathrm{~N}_{3} \mathrm{O}_{5} \mathrm{~S}$ \\
\hline$D_{\text {calc. }} / \mathrm{g} \mathrm{cm}^{-3}$ & 1.488 \\
\hline $\mathrm{m} / \mathrm{mm}^{-1}$ & 2.453 \\
\hline Formula Weight & 289.31 \\
\hline Colour & None \\
\hline Shape & $\mathrm{n} / \mathrm{a}$ \\
\hline Size $/ \mathrm{mm}^{3}$ & $\mathrm{n} / \mathrm{a} \times \mathrm{n} / \mathrm{a} \times \mathrm{n} / \mathrm{a}$ \\
\hline $\mathrm{T} / \mathrm{K}$ & $293(2)$ \\
\hline Crystal System & monoclinic \\
\hline Flack Parameter & $0.152(16)$ \\
\hline Hooft Parameter & $0.176(8)$ \\
\hline Space Group & $P 2_{1}$ \\
\hline$a / \AA$ & $6.9658(2)$ \\
\hline$b / \AA$ & $8.8494(2)$ \\
\hline$c / \AA ̊$ & $10.8590(3)$ \\
\hline$a /^{\circ}$ & 90 \\
\hline$b /^{\circ}$ & $105.295(3)$ \\
\hline$g /{ }^{\circ}$ & 90 \\
\hline$V / \AA^{3}$ & $645.67(3)$ \\
\hline Z & 2 \\
\hline$Z^{\prime}$ & 1 \\
\hline Wavelength/Å & 1.54184 \\
\hline Radiation type & $\mathrm{CuK}_{a}$ \\
\hline$Q_{\min } /^{\circ}$ & 4.221 \\
\hline$\left.Q_{\max }\right|^{\circ}$ & 66.479 \\
\hline Measured Refl. & 2890 \\
\hline Independent Refl. & 1576 \\
\hline Reflections with I > & 1558 \\
\hline $2(I)$ & \\
\hline$R_{\text {int }}$ & 0.0158 \\
\hline Parameters & 176 \\
\hline Restraints & 1 \\
\hline Largest Peak & 0.148 \\
\hline Deepest Hole & -0.151 \\
\hline GooF & 1.058 \\
\hline$w R_{2}$ (all data) & 0.0683 \\
\hline$w R_{2}$ & 0.0681 \\
\hline$R_{1}$ (all data) & 0.0255 \\
\hline
\end{tabular}




\section{Reduction to form 9 (3TC):}
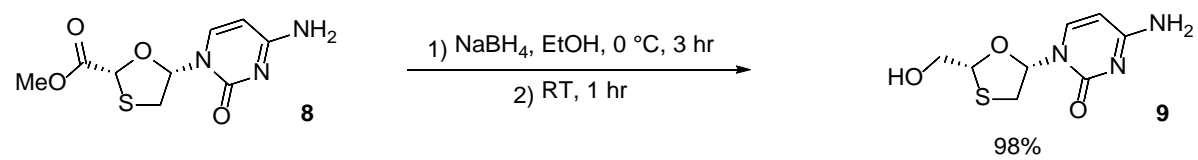

Figure S8: Synthesis of 3TC upon reduction of methyl ester 8.

\section{Typical Procedure for Synthesis of Lamivudine:}

Cytosinyl Oxathiolane 8 ( $2.62 \mathrm{~g}$, $87.6 \mathrm{wt} \%, 8.92 \mathrm{mmol}, 1$ equiv.) and ethanol ( $26 \mathrm{~mL}, 85 \%$ Biomatik) were combined in a round-bottom flask and cooled to $0{ }^{\circ} \mathrm{C}$ with an ice bath. Sodium borohydride $(618 \mathrm{mg}$, $16.3 \mathrm{mmol}, 1.83$ equiv., $>98 \%$ Sigma-Aldrich) was added. The reaction was stirred for $3 \mathrm{hr}$ at $0{ }^{\circ} \mathrm{C}$ and 1 $\mathrm{hr}$ at RT. The reaction was monitored for completion by TLC (20\% MeOH in DCM). The reduction was quenched by addition of $3 \mathrm{M} \mathrm{HCl}($ aq., $3.5 \mathrm{~mL}$ ) to reach $\mathrm{pH} 5-6$. After quench, the $\mathrm{pH}$ was adjusted to $\sim 11-12$ by addition of $50 \% \mathrm{NaOH}$ (aq., $525 \mu \mathrm{L}$ ). The reaction was monitored by quantitative NMR ( $98 \%$ assay yield). Solids were removed by filtration and the solids were rinsed twice with ethanol. The solvent was stripped from the filtrate by heating at $50{ }^{\circ} \mathrm{C}$ under vacuum to obtain a fine white powder matching reported values for $3 \mathrm{TC}\left(2.455 \mathrm{~g}, 81.5 \mathrm{wt} \%, 97.9 \%\right.$ yield). ${ }^{1} \mathrm{H}$ NMR $\left(600 \mathrm{MHz}, \mathrm{D}_{2} \mathrm{O} \_s a l t\right) \delta 7.89-$ $7.80(\mathrm{~m}, 1 \mathrm{H}), 6.17(\mathrm{dd}, \mathrm{z} J=5.5,4.3 \mathrm{~Hz}, 1 \mathrm{H}), 5.88(\mathrm{~d}, J=7.6 \mathrm{~Hz}, 1 \mathrm{H}), 5.22(\mathrm{dd}, J=4.5,3.4 \mathrm{~Hz}, 1 \mathrm{H}), 3.89(\mathrm{dd}, J=12.7$, $3.4 \mathrm{~Hz}, 1 \mathrm{H}$ ), 3.79 (dd, $J=12.7,4.6 \mathrm{~Hz}, 1 \mathrm{H}$ ), 3.44 (dd, $J=12.2,5.6 \mathrm{~Hz}, 1 \mathrm{H}$ ), 3.07 (dd, $J=12.2,4.2 \mathrm{~Hz}, 1 \mathrm{H}$ ). ${ }^{13} \mathrm{C} \mathrm{NMR}$ $\left(151 \mathrm{MHz}, \mathrm{D}_{2} \mathrm{O}\right.$ _salt) $\delta 166.06$ (s), 157.09 (s), 141.59 (s), 95.76 (s), 87.23 (s), 86.01 (s), 62.11 (s), 36.83 (s). HRMS: Expected $\mathrm{M}+\mathrm{H} \mathrm{m} / \mathrm{z} 230.0599$, Found: 230.0587.

\section{Reaction Sensitivities-Important Notes for Reproduction:}

- Reaction must be kept cold from time sodium borohydride is added. Byproduct is generated when reaction is run warm. 


\section{Lamivudine}

${ }_{\mathrm{S}}^{\cdots}{ }_{\mathrm{O}}^{\mathrm{O}}{ }_{\mathrm{N}} \mathrm{NOH}_{2}$
${ }^{1} \mathrm{H}$ NMR $\left(600 \mathrm{MHz}, \mathrm{D}_{2} \mathrm{O} \_\right.$salt) $\delta 7.89-7.80(\mathrm{~m}, 1 \mathrm{H}), 6.17(\mathrm{dd}, \mathrm{z} J=5.5,4.3 \mathrm{~Hz}, 1 \mathrm{H}), 5.88(\mathrm{~d}, J=7.6 \mathrm{~Hz}, 1 \mathrm{H}), 5.22(\mathrm{dd}, J=4.5,3.4 \mathrm{~Hz}, 1 \mathrm{H}), 3.89(\mathrm{dd}, J=12.7,3.4 \mathrm{~Hz}, 1 \mathrm{H}), 3.79$ $(\mathrm{dd}, J=12.7,4.6 \mathrm{~Hz}, 1 \mathrm{H}), 3.44$ (dd, $J=12.2,5.6 \mathrm{~Hz}, 1 \mathrm{H}), 3.07$ (dd, $J=12.2,4.2 \mathrm{~Hz}, 1 \mathrm{H})$.

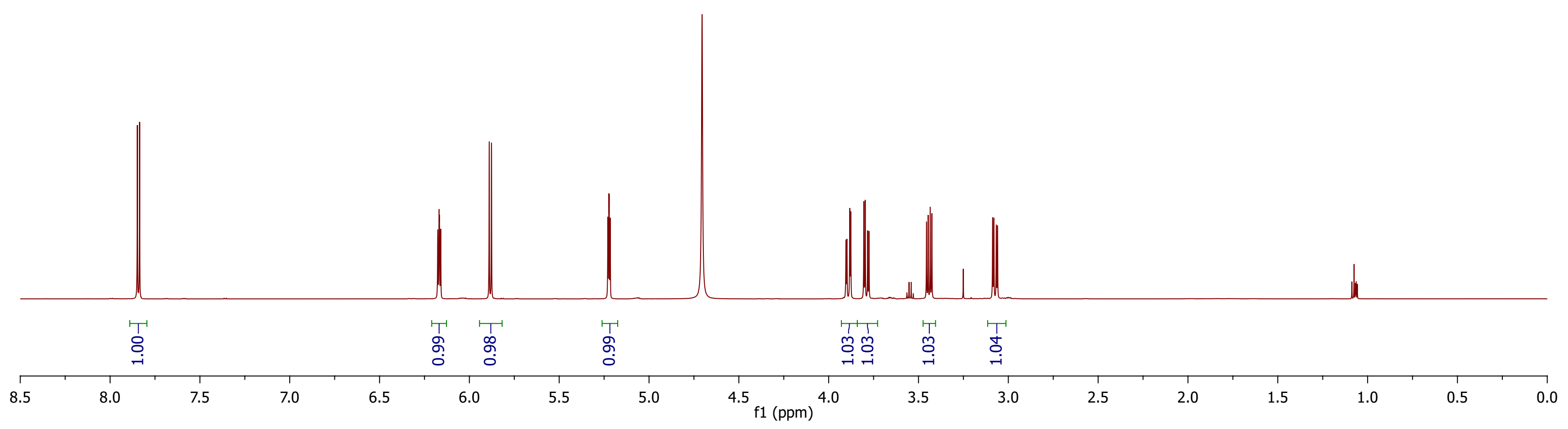


Lamivudine

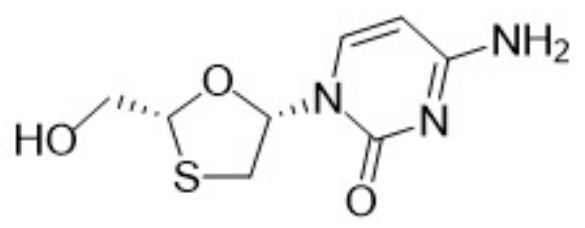

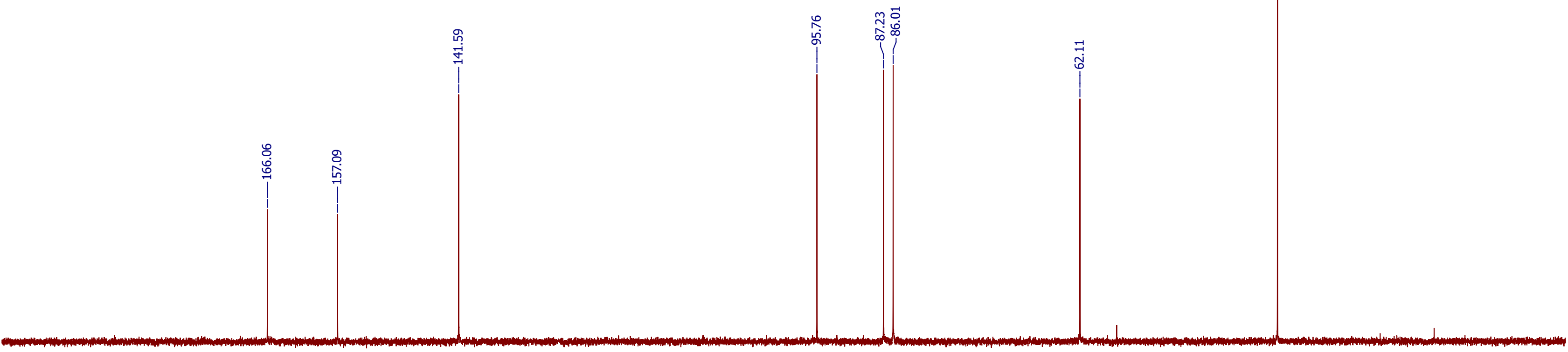


In Process Control at pH 12:

Quantitative NMR Assayed Against Acetonitrile

${ }_{\mathrm{S}}^{\cdots} \prod_{\mathrm{O}}^{\mathrm{N}}$

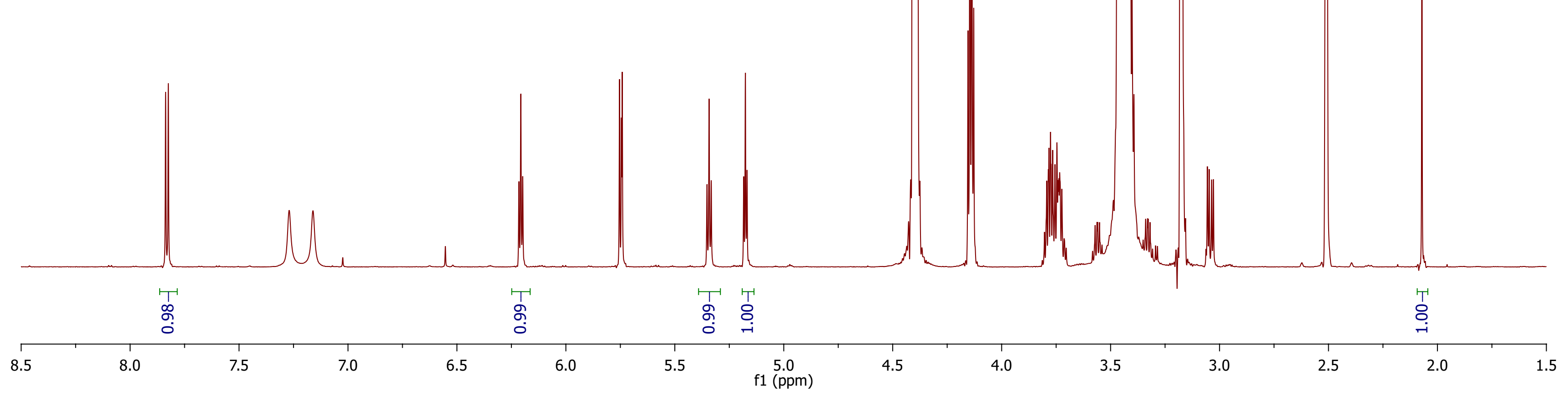


Sample Assayed for Purity by

Quantitative NMR: $82 \%$

$\left.{ }_{\mathrm{S}}^{\cdots}\right\rangle_{\mathrm{O}}^{\mathrm{O}} \prod_{\mathrm{N}}^{\mathrm{NH}_{2}}$

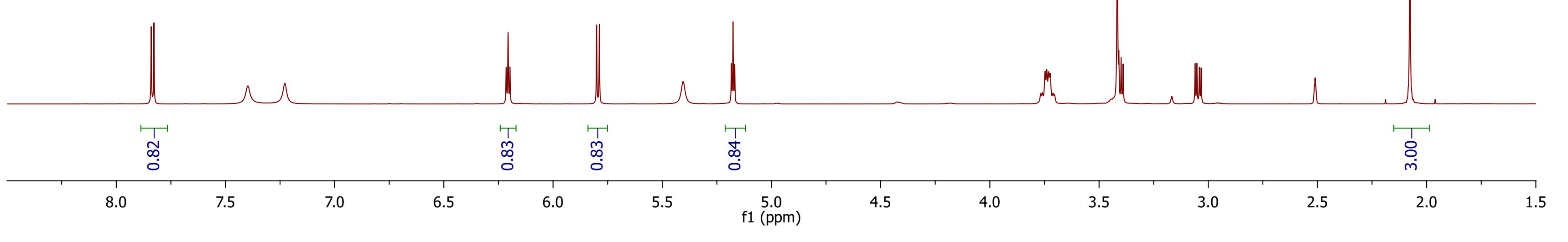


Temperature Dependence:

$50{ }^{\circ} \mathrm{C}$ Before Quench

${ }_{\mathrm{HO}}^{\prime \prime}>_{\mathrm{O}}{ }_{\mathrm{O}}{ }_{\mathrm{N}} \mathrm{NH}_{2}$

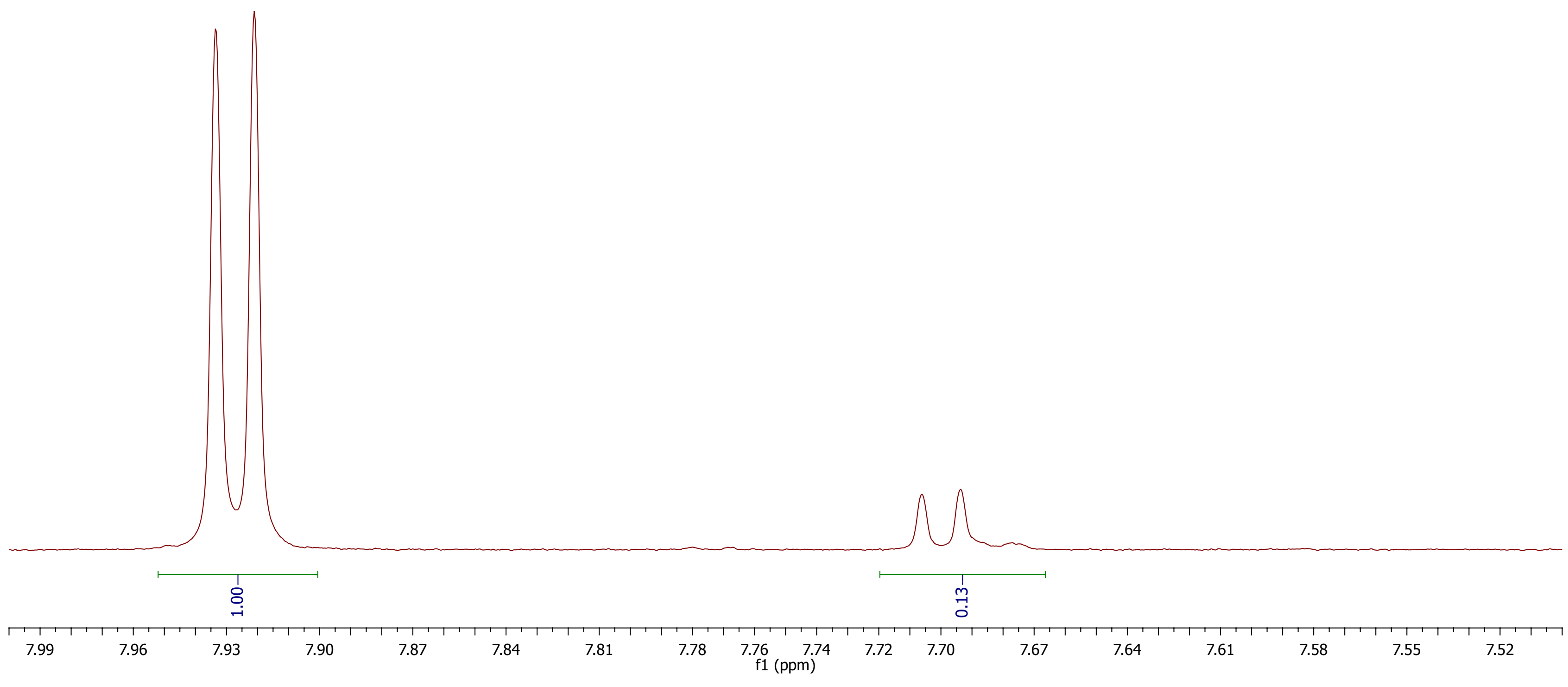


Temperature Dependence:

$20^{\circ} \mathrm{C}$ Before Quench

${ }_{\mathrm{S}}^{\prime \prime .} \mathrm{C}_{\mathrm{O}}^{\mathrm{O}}$

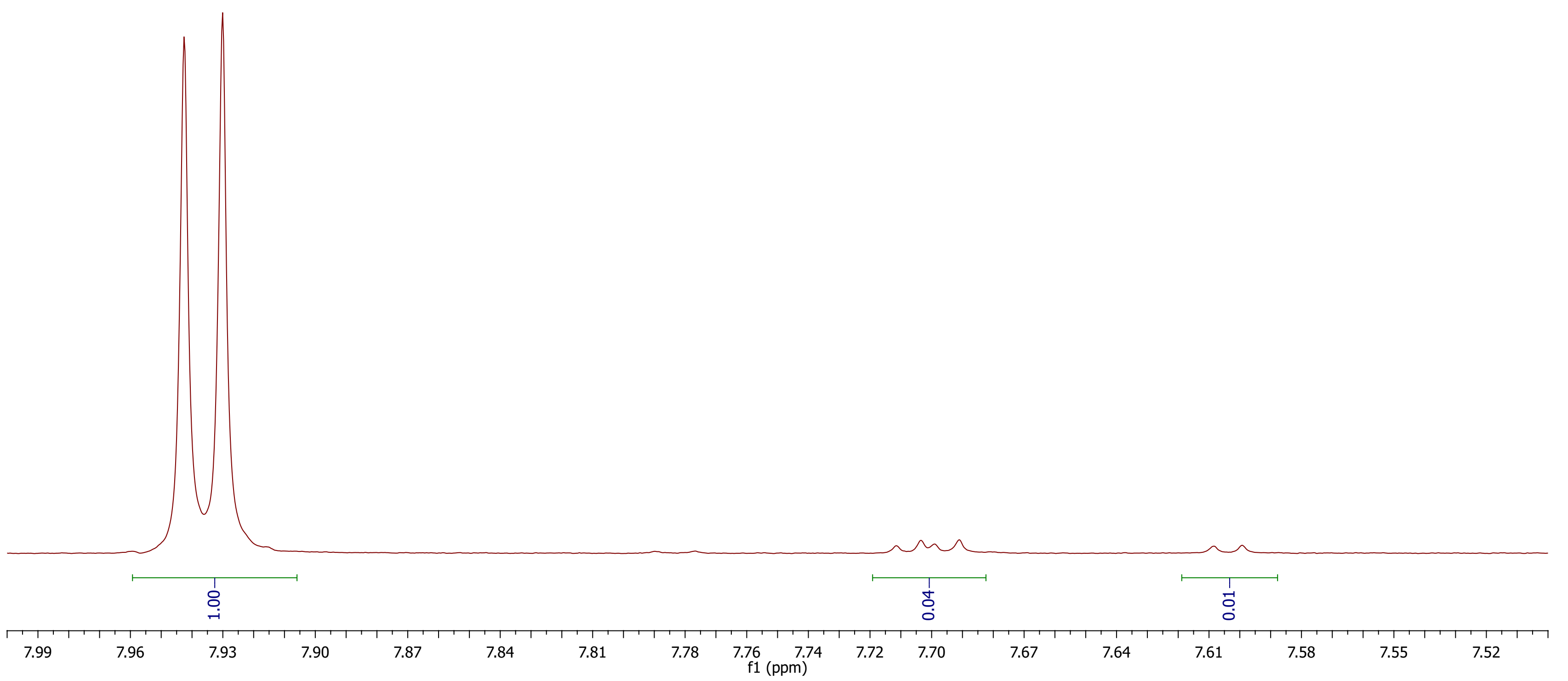


Temperature Dependence: $0{ }^{\circ} \mathrm{C}$ Before Quench

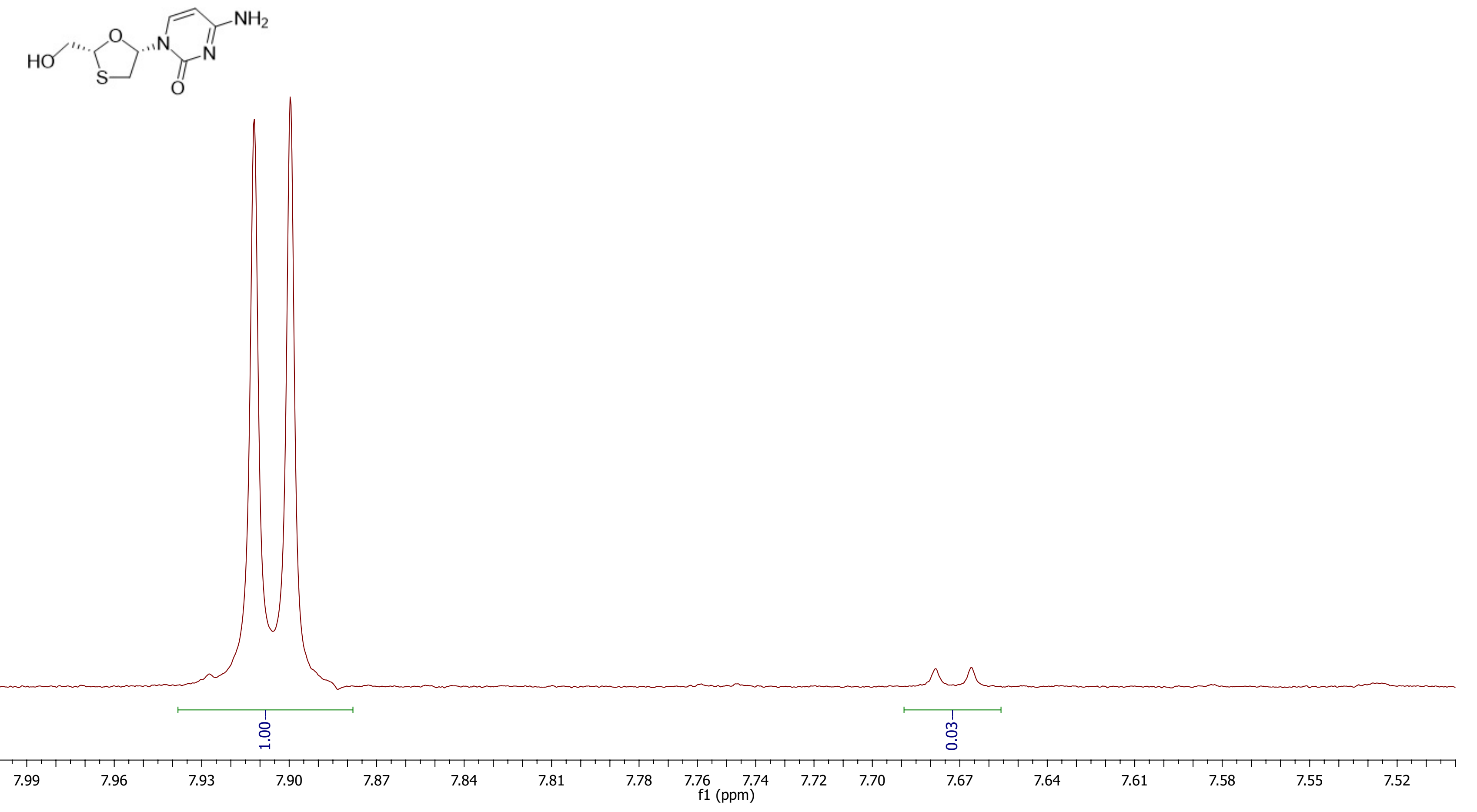


r200204r03\#2-50 RT: $0.01-0.40$ AV: 49 NL: 4.19E7

T: FTMS + p NSIFull ms [100.00-1000.00]

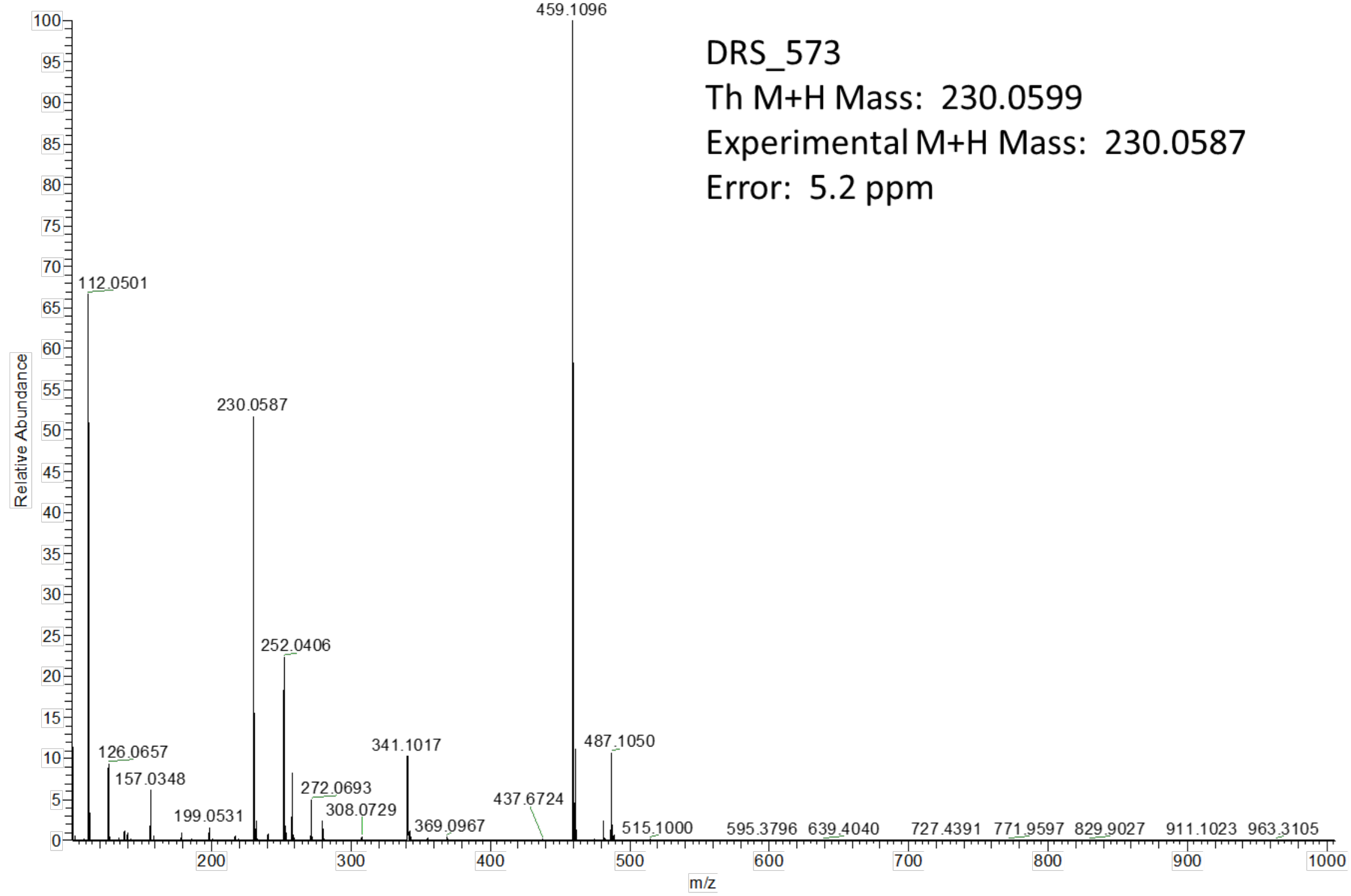

DRS_573

Experimental M+H Mass: 230.0587

Error: $5.2 \mathrm{ppm}$ 


\section{SFC Analysis Conditions:}

Column: ChiralPak IA, $4.6 \mathrm{~mm}$ ID X $250 \mathrm{mmL}, 3 \mathrm{uM}$ particles Mobile Phase: $20 \%$ Methanol (0.5\% DEA) in $\mathrm{CO}_{2}$

Flow Rate: $2.0 \mathrm{~mL} / \mathrm{min}$

Detection: $275 \mathrm{~nm}$

\section{Retention Times:}

Cytosine

8 (2R, 5S-desired)

8 (2S, 5R-enantiomer)

8 (2S, $5 S$ and $2 R, 5 R$-trans diastereomers)

9 (Lamivudine, 2R, 5S)

9 (enantiomer, 2S, 5R)

9 (trans diastereomers)
$5.2 \mathrm{~min}$

$5.4 \mathrm{~min}$

$6.5 \mathrm{~min}$

7.0 and $7.7 \mathrm{~min}$

$6.9 \mathrm{~min}$

$7.9 \mathrm{~min}$

9.2 and $14.6 \mathrm{~min}$ 
A Mixture of 3TC Stereoisomers Spiked with Authentic 3TC

\section{SFC Report}

Data file:

Sample name:

Description:

Sample amount:

Instrument:

Injection date:

Acq. method:

Analysis method:

Last changed:
C: Users $\backslash P$ ublic \Documents $\backslash$ ChemStation $\backslash 1 \backslash D a t a \backslash S 0112120 \backslash 3 T C$ 2020-01-22 0845-16\007-D1F-C1-3TC-Spike.D

3TC-Spike

Old-Sample

0.000

Sample type:

Sample

SFC

1/22/2020 10:51:43 AM

Location:

3TC Chiral.M

3TC Chiral.M

1/24/2020 3:22:07 PM
Injection: $\quad 1$ of 1

Injection volume: $\quad 5.000$

Acq. operator: $\quad$ SYSTEM

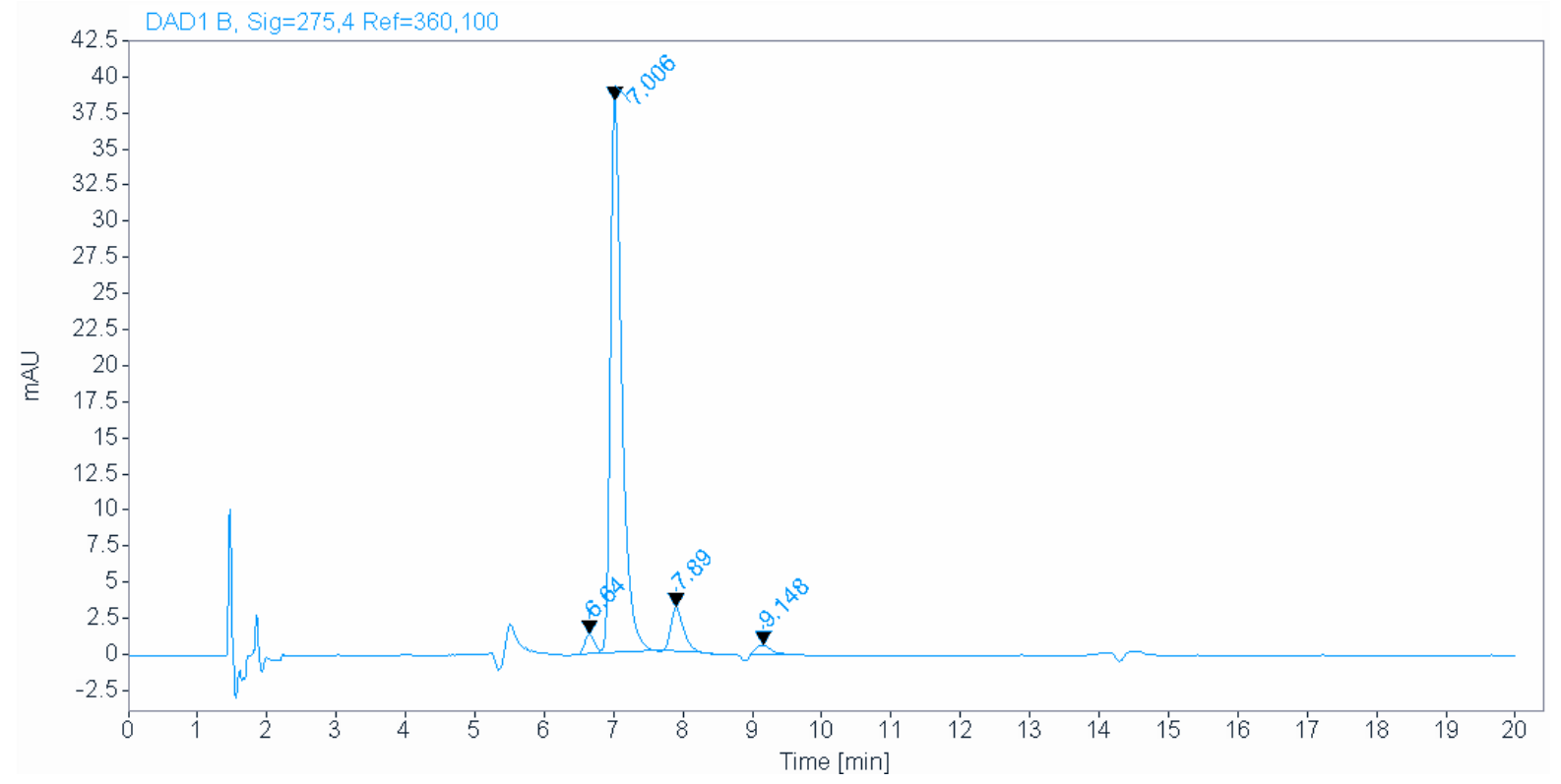

Signal: $\quad$ DAD1 B, Sig $=275,4$ Ref $=360,100$

$\begin{array}{crrrr}\text { RT [min] Type } & \text { Width [min] } & \text { Area } & \text { Height } & \text { Area\% Name } \\ \text { 6.640 MF } & 0.1676 & 14.1710 & 1.4089 & 2.7860 \\ \text { 7.006 FM } & 0.1920 & 441.1843 & 38.2873 & 86.7370 \\ 7.890 \mathrm{BB} & 0.1985 & 41.2003 & 3.1105 & 8.1000 \\ 9.148 \mathrm{MM} & 0.2785 & 12.0905 & 0.7237 & 2.3770 \\ & \text { Sum } & 508.6461 & & \end{array}$




\section{TC Made from (S)-2-Chloropropionic Acid}

\section{SFC Report}

Data file:

Sample name:

Description:

Sample amount:

Instrument:

Injection date:

Acq. method:

Analysis method:

Last changed:
C: Users $\backslash P$ ublic \Documents $\backslash$ ChemStation $\backslash 1 \backslash D a t a \backslash S 0112120 \backslash 3 T C$ 2020-01-22 0845-16\005-D1F-E2-DRS-573-3TC.D

DRS-573-3TC

$\sim 2.5 \mathrm{mg} / \mathrm{mL}$ in $\mathrm{MeOH}$

0.000

Sample type:

Sample

SFC

1/22/2020 10:09:57 AM

Location:

Injection:

D1F-E2

3TC Chiral.M

3TC Chiral.M

1/24/2020 3:22:07 PM

Acq. operator: $\quad$ SYSTEM

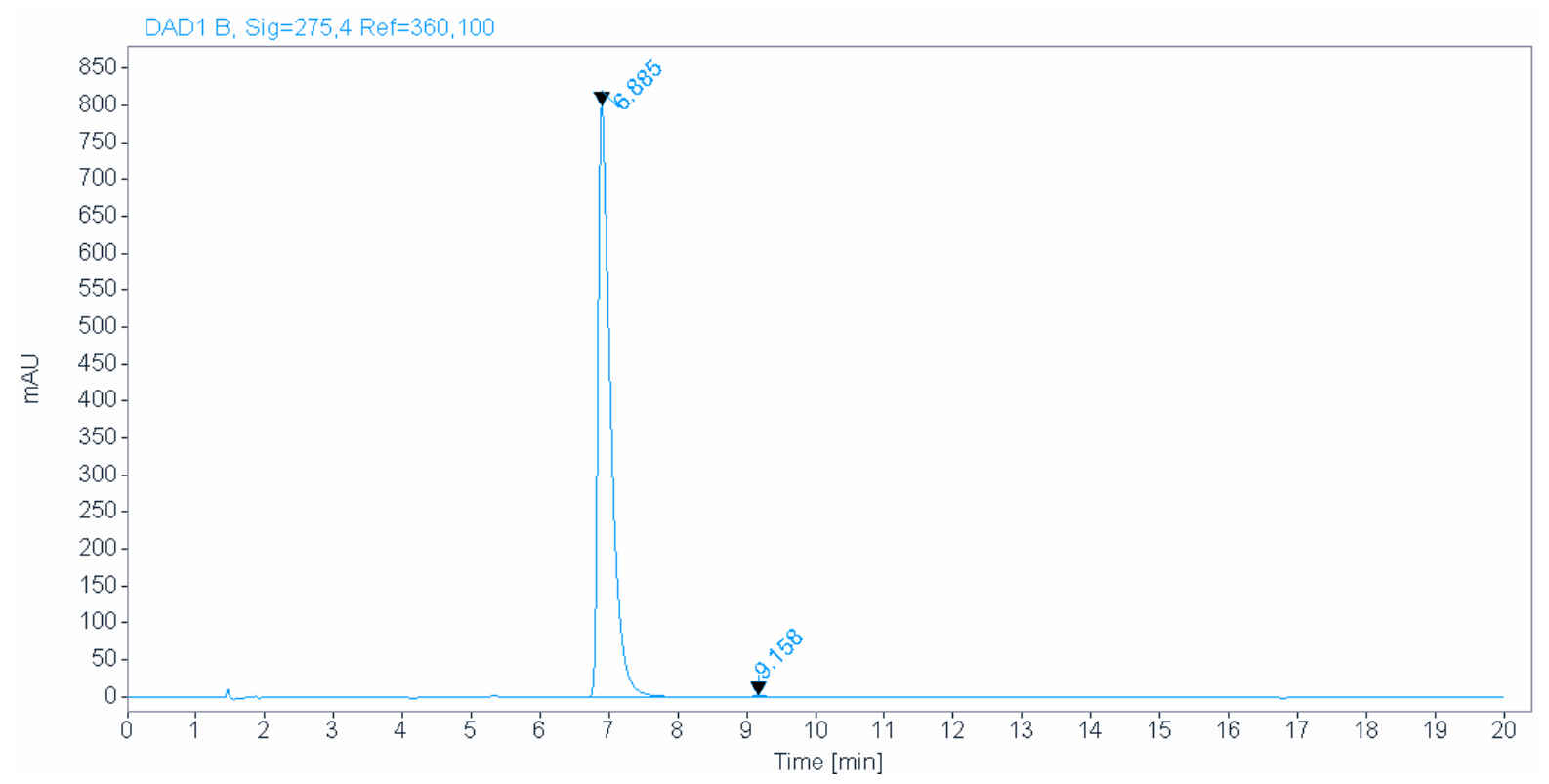

Signal: $\quad$ DAD1 B, Sig $=275,4$ Ref $=360,100$

RT [min] Type Width [min]

Area

Height

Area\% Name

$6.885 \mathrm{BB}$

0.1896

10269.1777

801.0740

99.6123

$9.158 \mathrm{BB}$

0.2412

39.9683

2.4903

0.3877

Sum 10309.1460 